AMANDA SOUZA DE PAULA

\title{
UM ESTUDO COMPARATIVO ENTRE OS SISTEMAS OFDM E SCCP
}

Dissertação apresentada à Escola Politécnica da Universidade de São Paulo para obtenção do Título de Mestre em Engenharia Elétrica.

São Paulo

2010 
AMANDA SOUZA DE PAULA

\section{UM ESTUDO COMPARATIVO ENTRE OS SISTEMAS OFDM E SCCP}

Dissertação apresentada à Escola Politécnica da Universidade de São Paulo para obtenção do Título de Mestre em Engenharia Elétrica.

Área de Concentração:

Sistemas Eletrônicos

Orientador:

Prof. Dr. Cristiano Magalhães Panazio

São Paulo

2010 
Este exemplar foi revisado e alterado em relação à versão original, sob responsabilidade única do autor e anuência do orientador.

São Paulo, 12 de fevereiro de 2010

Assinatura do autor

Assinatura do orientador

Paula, Amanda Souza de

Um estudo comparativo entre os sistemas OFDM e SCCP/A.S. de Paula.

- São Paulo, 2010.

$119 \mathrm{p}$.

Dissertação (Mestrado) - Escola Politécnica da Universidade de São Paulo. Departamento de Engenharia de Telecomunicações e Controle.

1. Codificação 2. Equalização 3. Modulação digital I. Universidade de São Paulo. Escola Politécnica. Departamento de Engenharia de Telecomunicações e Controle II.t 


\section{Resumo}

Este trabalho trata da comparação entre os sistemas OFDM (orthogonal frequency division multiplexing) e SCCP (single carrier with cyclic prefix). Tais sistemas são analisados em função da ordem da modulação, da sensibilidade em relação à taxa de codificação de canal e do tipo de entrelaçamento utilizado. A comparação é efetuada através da adoção de um arcabouço analítico que provê uma abordagem universal para o tratamento de ambos os sistemas.

São obtidos resultados teóricos, explorando características de convexidade de funções, e resultados a partir de simulação Monte Carlo.

Os sistemas são comparados, principalmente, em termos de taxa de erro de bit (BER, do inglês, bit-error rate). Entretanto, também são realizadas comparações em termos de probabilidade de outage por cutoff rate e da relação sinal-ruído (SNR, do inglês, signal to noise ratio) efetiva na saída do equalizador.

Finalmente, os sistemas ainda são avaliados quanto à sensibilidade a erros de estimação de canal. 


\section{Abstract}

This work deals with the comparison between the orthogonal frequency division multiplexing (OFDM) and single carrier with cyclic prefix (SCCP). These systems are analyzed as a function of the modulation order, the sensitiveness to the channel coding rate and to the interleaver configuration. The comparison is accomplished by means of a system model that leads to an universal framework to both analyzed systems.

Theoretical results are obtained exploring convexity properties of functions. In addition, some results provided by Monte Carlo simulation are also presented to complement the analysis.

The systems are compared mainly in terms of the bit error rate (BER). Moreover, comparisons in terms of outage probability obtained by the cutoff rate and in terms of the effective signal to noise ratio (SNR) at the equalizer output are also provided.

Finally, the systems are compared when channel estimation errors are present at the receiver. 


\section{Agradecimentos}

Agradeço, antes de tudo, ao amigo e professor Cristiano Panazio. Sou particularmente grata pela confiança em mim depositada, pelo constante apoio, por sua imensa dedicação. Realmente, me sinto privilegiada por ter tido a chance de desenvolver esse longo trabalho sob sua orientação.

Aos professores do LCS da Escola Politécnica da USP, pela oportunidade de estudar os assuntos que mais me empolgam da forma mais interessante possível. Em especial, agradeço à Prof. Maria Miranda que, além das contribuições acadêmicas, sempre me dedicou uma incrível atenção.

Agradeço ao Prof. Phillip Burt e ao Prof. Renato Lopes pelas valiosas sugestões dadas ainda no período de qualificação e que, certamente, contribuíram para a elevação do nível da dissertação.

Agradeço às minhas amigas-irmãs recifenses, Clarissa e Natália, que mesmo à distância, sempre conseguiram me alegrar e me apoiar.

Aos meus amigos da vida paulistana: Bruno, Flávio, Laércio, Lucas, Leonardo e Marcelo. Além das proveitosas discussões técnicas, sem eles o cotidiano na USP não teria sido tão agradável.

À minha família pelo apoio e amor a mim dedicados. O agradecimento se estende às minhas queridas primas, tias, avós... Mas eu agradeço, sobretudo, aos meus amados irmãos, Patrícia e Victor, e aos meus amados pais, Márcia e Jairo, que sempre souberam me inspirar e me encorajar ao longo de toda a minha vida. A eles dedico essa dissertação. 


\section{Lista de Símbolos}

$\mathcal{A}, \mathcal{B}, \cdots \quad$ Matriz no domínio da frequência

$\mathcal{A}_{k, m} \quad$ Elemento na $k$-ésima linha e $m$-ésima coluna da matriz $\mathcal{A}$

$\mathbf{A}, \mathbf{B}, \cdots$ Vetor no domínio da frequência

$A_{k} \quad k$-ésimo elemento do vetor $\mathbf{A}$

$\tilde{\mathcal{A}}, \tilde{\mathcal{B}}, \cdots \quad$ Matriz no domínio do tempo

a,b, $\cdots$ Vetor no domínio do tempo

$\mathcal{F}$ Matriz de Fourier

W Vetor de coeficientes do equalizador

$\mathbf{X}$ Vetor de dados no domínio na frequência

$\boldsymbol{v}$ Vetor ruído branco gaussiano no domínio do tempo

$\Upsilon$ Vetor ruído branco gaussiano no domínio da frequência

h Vetor resposta impulsiva do canal

$\mathbf{H}$ DFT de $\mathbf{h}$

$\mathcal{H}$ Matriz de convolução do canal

$\mathcal{H}_{c} \quad$ Matriz de convolução circular do canal

$\boldsymbol{I}_{N} \quad$ Matriz identidade de ordem $N$

harmmean $\{\cdot\} \quad$ Operador média harmônica

geomean $\{\cdot\} \quad$ Operador média geomátrica

$\mathrm{E}\{\cdot\} \quad$ Operador esperança

$\{\cdot\}^{*}$ Operador conjugado

$\{\cdot\}^{T} \quad$ Operador matriz transposta

$\{\cdot\}^{H}$ Operador matriz conjugada transposta

$\{\cdot\} \circ\{\cdot\} \quad$ Produto de Hadamard

$X \sim \operatorname{eN}\left(\mu, \sigma^{2}\right) \quad$ Variável aleatória circular gaussiana com média $\mu$ e variância $\sigma^{2}$ 


\section{Lista de Abreviações}

BER Taxa de erro de bit (Bit Error Rate)

BICM Bit Interleaved Coded Modulation

CP Prefixo cíclico (Cyclic Prefix)

DFE Equalizador com decisão realimentada (Decision Feedback Equalizer)

DFT Transformada discreta de Fourier (Discrete Fourier Transform)

IDFT Inversa da transformada discreta de Fourier (Inverse Discrete Fourier Transform)

IBI Interferência interbloco (Interblock Interference)

ISI Interferência intersimbólica (Intersymbol Interference)

LE Equalizador linear (Linear Equalizer)

MF Filtro Casado (Matched Filter)

MMSE Erro quadrático médio mínimo (Minimum Mean Square Error)

MSE Erro quadrático médio (Mean Square Error)

OFDM Orthogonal Frequency Division Multiplexing

PEP Pairwise Error Probability

QAM Modulação de amplitude em quadratura (Quadrature Amplitude Modulation)

QPSK Quatenary Phase Shift Modulation

SCCP Portadora única com prefixo cílico (Single Carrier with Cyclic Prefix)

SER Taxa de erro de símbolo (Symbol error rate)

SNR Relação sinal-ruído (Signal to noise ratio)

TCM Modulação codificada por treliça (Trellis Coded Modulation)

UW Unique Word

ZF Zero-Forcing 


\section{Sumário}

1 Introdução $\quad \mathbf{1}$

1.1 Contribuições . . . . . . . . . . . . . . . . . . . 6 6

1.2 Organização da dissertação . . . . . . . . . . . . . . . . . . 6

2 Transmissão multiportadora $\quad 9$

2.1 Princípios do sistema OFDM . . . . . . . . . . . . . . . . . 9

2.2 Ortogonalidade em canais com multipercurso . . . . . . . . . . . . 11

2.3 Prefixo cíclico . . . . . . . . . . . . . . . . . . . 12

2.4 Abordagem universal . . . . . . . . . . . . . . . . . 15

3 Equalização no domínio da frequência $\quad \mathbf{1 7}$

3.1 Modelo do sistema . . . . . . . . . . . . . . . . . 17

3.2 Sistema OFDM . . . . . . . . . . . . . . . . . . 19

3.3 Sistema SCCP . . . . . . . . . . . . . . . . . . 20

3.3 .1 Equalização linear . . . . . . . . . . . . . . . 20

3.3 .2 Equalização com decisão realimentada . . . . . . . . . . . . 22

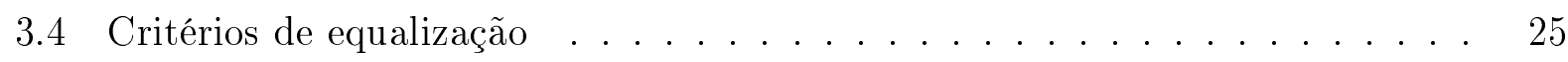

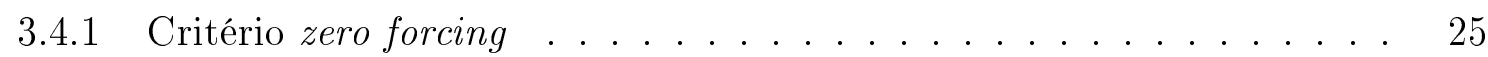

3.4 .2 Critério do mínimo erro quadrático médio . . . . . . . . . . 25

4 Efeitos da ordem da modulação

4.1 Derivação de expressões de BER . . . . . . . . . . . . . . . . . 33

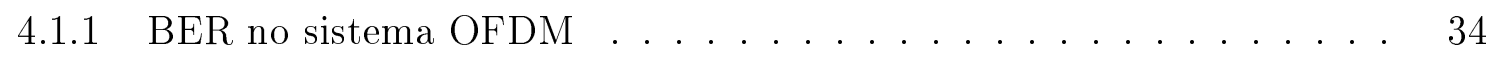

4.1 .2 BER no sistema SCCP . . . . . . . . . . . . . . 35

4.2 Comparação de desempenho . . . . . . . . . . . . . . . . 35

4.2 .1 OFDM vs LE-SCCP . . . . . . . . . . . . . . 36

4.2 .2 OFDM vs DFE-SCCP ........................... 41 
5 Sensibilidade em relação à taxa de codificação $\quad 49$

5.1 Capacidade de Shannon . . . . . . . . . . . . . . . . . . 49

5.2 Análise através da cutoff rate . . . . . . . . . . . . . . . 51

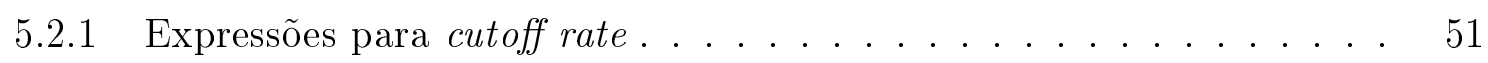

5.2 .2 Resultados . . . . . . . . . . . . . . . . 57

6 Efeitos do entrelaçador $\quad 61$

6.1 Entrelaçamento . . . . . . . . . . . . . . . . . 62

6.1.1 Entrelaçamento regular . . . . . . . . . . . . . 62

6.1.2 Entrelaçamento modular . . . . . . . . . . . . . . 63

6.1.3 Entrelaçamento aleatório . . . . . . . . . . . . . 66

6.2 Canal seletivo em frequência e estático . . . . . . . . . . . . . 67

6.2.1 Efeito do entrelaçamento . . . . . . . . . . . . 67

6.2 .2 Comparação de desempenho . . . . . . . . . . . . . . . 73

6.3 Canais seletivos em frequência com desvanecimento por blocos . . . . . . . . 75

6.3.1 Impacto da escolha do entrelaçador . . . . . . . . . . . . . 77

6.3.2 Análise com modulação 16-QAM . . . . . . . . . . . 80

6.3.3 Taxa de codificação . . . . . . . . . . . . . . . 82

7 Comparação através da SNR efetiva $\quad 85$

7.1 Definição da SNR efetiva . . . . . . . . . . . . . . . . 86

7.2 BER para sistemas codificados . . . . . . . . . . . . . 86

7.3 Comparação entre os sistemas OFDM e LE-SCCP . . . . . . . . . . . . . . 90

7.4 Comparação entre os sistemas OFDM e DFE-SCCP . . . . . . . . . . . . 92

8 Sensibilidade a erros de estimação de canal $\quad 95$

8.1 Efeito do erro de estimação de canal no sistema OFDM . . . . . . . . . . . 95

8.2 Efeito do erro de estimação de canal no sistema SCCP . . . . . . . . . . 98

8.3 Comparação OFDM vs SCCP . . . . . . . . . . . . . . . . . 100

9 Conclusões e Perspectivas 103

$\begin{array}{ll}\text { Apêndice } & 107\end{array}$

A Tratamento de desigualdades: Hardy, Littlewood e Pólya 107

B Análise da função $b(y) \quad 111$ 
Referências Bibliográficas 


\section{Capítulo 1}

\section{Introdução}

A busca por sistemas de comunicações que utilizem eficientemente o canal de transmissão disponível não é uma tarefa recente. Ainda na época da comunicação via telégrafo, na segunda metade do século XIX, o engenheiro francês Émile Baudot desenvolveu um sistema de transmissão aplicando multiplexação no tempo (TDM, do inglês, time division multiplexing) [1]. Com esse sistema, era possível utilizar uma única linha de transmissão para transmitir mensagens de quatro teleimpressoras diferentes.

A primeira demonstração de transmissão por multiplexação na frequência (FDM, do inglês, frequency division multiplexing) foi realizada em 1910 pelo engenheiro George Squier [2]. Em sua demonstração, Squier utilizou uma única linha telefônica para transmitir dois sinais de voz simultaneamente. Em 1918 surge a primeira aplicação comercial de sistemas utilizando FDM, quando a AT\&T lança seu sistema FDM com cinco canais.

Nos sistemas FDM, o espectro é dividido em canais sem sobreposição de frequência. Para que os canais sejam adequadamente separados na recepção, é necessário que filtros sintonizadores com banda de transição estreita sejam aplicados. Se tais filtros não satisfazem essa condição, é necessário introduzir uma banda de guarda entre canais adjacentes, fato que faz com que o espectro não seja utilizado eficientemente.

Tal limitação dos sistemas FDM é superada nos sistemas OFDM (do inglês, orthogonal frequency division multiplexing). Nesses sistemas, os sinais são transmitidos em subcanais, também referidos como subportadoras, que admitem sobreposição tanto no tempo como na frequência. A recuperação dos sinais transmitidos em cada subcanal é promovida explorando uma característica especial imposta nas subportadoras: a ortogonalidade.

A idéia de transmissão por multiportadoras ortogonais foi proposta por Chang em 1966 [3]. Ainda na década de 60, algumas primeiras aplicações de cunho predominantemente 
militar foram implementadas com sucesso. Nesse contexto foram desenvolvidos os modems KINEPLEX, KATHRYN e ANDEFT [4], [5].

Esses primeiros sistemas foram implementados utilizando-se um oscilador local para cada subportadora. Há uma série de desvantagens relacionadas à complexidade e custo inerente a esse tipo de implementação, o que impedia a disseminação desta em contextos mais amplos.

A publicação de Cooley e Tukey em 1965 [6] trouxe novas perspectivas para a transmissão multiportadora. No algoritmo proposto em [6], o número de multiplicações necessárias para o cálculo de uma transformada discreta de Fourier (DFT, do inglês, discrete Fourier transform) de uma sequência de $N$ elementos foi reduzido de $N^{2}$ para $N \log _{2} N$.

A partir do artigo de Cooley e Tukey, em 1971, Weinstein e Ebert propuseram uma implementação digital do sistema OFDM [7]. Como será detalhado no capítulo 2, nesse trabalho, os autores mostraram ser possível gerar amostras do sinal OFDM aplicando a transformada inversa discreta de Fourier (IDFT, do inglês, inverse discrete Fourier transform) na sequência de dados a ser transmitida. A partir de então, a implementação de sistemas OFDM passa a ser analisada como uma alternativa viável à transmissão serial, i.e., por portadora única.

A primeira aplicação comercial e de maior impacto do OFDM surge no final da década de 80 com o sistema ADSL (do inglês, assymetric digital subscriber line) [8]. Tal sistema permitiu alcançar altas taxas de transmissão de dados pela linha telefônica dos assinantes, taxas estas muito maiores que os sistemas concorrentes da época.

Desde então, o sistema OFDM tem estado em grande evidência e vem sendo adotado por outros importantes padrões de comunicação digital. Além do sistema ADSL, dentre suas principais aplicações estão a TV digital (DVB-T [9], ISDB-T [10] e ISDB-Tb), sistemas de comunicação pela linha elétrica (PLC, do inglês, power line communication), redes locais de computadores sem fio (IEEE $802.11 \mathrm{a} / \mathrm{g} / \mathrm{n}$ ) [11] e sistema de rádio difusão digital (DAB, do inglês, digital audio broadcast) [12].

Algumas características do sistema OFDM justificam sua rápida disseminação nos últimos anos. A principal delas reside no fato de que, desde que a transmissão se dê com prefixo cíclico (CP, do inglês, cyclic prefix) suficiente, o sinal recebido em cada subportadora é livre de interferência intersimbólica (ISI, do inglês, intersymbol interference). Dessa forma, esse sinal corresponde exatamente ao sinal transmitido, multiplicado apenas por uma constante complexa. Essa característica elimina uma das maiores dificuldades na transmissão por portadora única: a demanda por equalizadores, eventualmente longos, em canais dispersivos. No sistema OFDM, a ausência de ISI faz com que a equalização se limite a uma multiplicação complexa para que a fase e a magnitude do sinal seja corrigida.

Um segundo fator motivador do uso do OFDM consiste no fato de que o conhecimento do canal na trasmissão permite aplicar o algoritmo de alocação de potência de waterfilling 
[13] e, dessa forma, o desempenho do sistema pode melhorar significativamente. Apesar de também ser possível aplicar algoritmos de waterfilling em outros sistemas, tal como o sistema de portadora única, a técnica é consideravelmente mais complexa do que nos sistemas OFDM [14] ou resulta em ganhos menores [15]. Em função dos resultados já publicados, não restam dúvidas de que o OFDM com waterfilling é a melhor solução. Contudo, no contexto em que o conhecimento do canal não se encontra disponível no transmissor, e em que a potência alocada para toda a faixa de transmissão é uniforme, ainda há margem para questionamento. É neste contexto que se situa esta dissertação.

Nesse contexto, como principal problema do OFDM, pode-se citar o fato de que cada símbolo é transmitido em apenas uma subportadora. Caso o canal gere um desvanecimento profundo na frequência dessa subportadora, o símbolo a ela associado será muito provavelmente corrompido. Se a informação que aquele símbolo carrega não for também enviada em outras subportadoras não afetadas pelo desvanecimento, ela estará comprometida.

Por outro lado, outros tipos de modulação são inerentemente robustos à seletividade em frequência, pois cada símbolo tem seu conteúdo espalhado por toda a faixa de transmissão. A modulação por portadora única é um exemplo clássico de modulação com tal propriedade.

Historicamente, um dos maiores problemas na transmissão por portadora única residia na complexidade envolvida na equalização. A presença da ISI, inexistente no sistema OFDM, demanda a utilização de tais mecanismos de equalização que podem ser custosos e de difícil implementação.

Entretanto, como mostrado em [16], o uso do prefixo cíclico permite que a equalização linear do sinal de portadora única seja feita no domínio da frequência, do mesmo modo como é feito no OFDM. Tal de equalizador é denotado por one-tap equalizer e o sistema de portadora única linearmente equalizado, em conjunto com o prefixo cíclico, dá origem ao LE-SCCP (do inglês, linear equalized single carrier with cyclic prefix).

Além da equalização linear, uma alternativa bem difundida para a equalização de sinais de portadora única é o equalizador com decisão realimentada (DFE, do inglês, decision feedback equalizer). O DFE foi proposto em [17] e desde então vem sendo amplamente utilizado para combater a ISI gerada em canais fortemente seletivos em frequência. Em [18] é apresentada uma forma de implementar tal equalizador também no domínio da frequência. Nessa primeira proposta, a ideia consistia em transmitir blocos sem extensão cíclica e combater a interferência inter-blocos através de técnicas do tipo overlap-save [19]. Posteriormente, como descrito em [20] e [21], sugiram algumas propostas de implementações do DFE no domínio da frequência também com extensão cíclica. Tais implementações permitem que o filtro direto seja implementado com a estrutura do one-tap equalizer.

Em [22], são destacadas as várias similaridades entre os sistemas OFDM, LE-SCCP e 
DFE com extensão cíclica. Tais similaridades levaram a uma série de trabalhos visando estabelecer comparações de desempenho entre esses sistemas.

Alguns desses trabalhos de comparação se restringem a aplicações sem codificação de canal. Esse é o caso de [23], onde é mostrado matematicamente que se for utilizada modulação QPSK, o sistema OFDM sempre apresenta pior desempenho, em termos de taxa de erro de bit (BER, do inglês, bit error rate), que o sistema LE-SCCP. Em [24] é mostrado que tal conclusão não é válida se a modulação 16-QAM for utilizada. Nessa dissertação, a comparação entre sistemas não-codificados será estendida aos sistemas DFE-SCCP e a modulações de ordens mais elevadas.

Entretanto, como já foi enfatizado, o sistema OFDM depende da codificação de canal para obter bom desempenho. Portanto, é nesse contexto que se insere a maioria das publicações. Em [25], é considerada transmissão em canais seletivos em frequência com desvanecimento Rayleigh por blocos e é empregada uma análise de cutoff rate, na qual se mostra que, para sistemas codificados com taxas menores do que $R=1 / 2$, os sistemas OFDM e LE-SCCP tendem a apresentar o mesmo desempenho em termos de probabilidade de outage. Já para taxas de codificação mais elevadas, o LE-SCCP supera o OFDM.

O resultado em [25] corrobora os resultados mostrados em [22], que indicam a degradação do desempenho do OFDM em relação ao LE-SCCP trazida pelo aumento da taxa de codificação.

Ainda em [22] é observado que, com o aumento do nível da modulação, o LE-SCCP se degrada em relação ao OFDM. Esse fato é constatado estabelecendo a comparação entre os sistemas em dois cenários distintos: modulação QPSK e 64-QAM. Enquanto ambos os sistemas apresentam praticamente o mesmo desempenho no contexto de transmissão QPSK, no caso da modulação 64-QAM, o OFDM passa a apresentar desempenho consideravelmente superior. Tal comportamento também foi observado em [26].

Apesar dessas evidências que indicam uma degradação de desempenho dos sistemas SCCP com o aumento da ordem da modulação, ainda não havia sido publicado um trabalho mais criterioso a respeito desse tema. Essa lacuna na literatura motivou uma análise dedicada ao efeito da ordem da modulação na comparação entre os sistemas. Então, ao longo deste trabalho, foi desenvolvido um método analítico para avaliar tal efeito. No caso dos sistemas não-codificados, a comparação foi estabelecida em termos de BER, e para o caso de sistemas codificados, a probabilidade de outage por cutoff rate foi definida como parâmetro de comparação.

Além da BER e da cutoff rate, questões relacionadas à diversidade constituem um terceiro universo na qual a comparação pode ser inserida. Entende-se por diversidade a técnica de transmitir a mesma informação por diferentes canais e, dessa forma, aumentar a probabi- 
lidade de sucesso, pois aumenta-se a chance de ter condições favoráveis para a transmissão. Em relação ao sistema SC, há uma série de trabalhos demonstrando que tais sistemas conseguem explorar a diversidade em frequência do canal mesmo sem código. Por exemplo, em [27] é mostrado que o SC com equalização máxima verossimilhança (ML, do inglês, maximum likelihood) consegue explorar a diversidade em frequência do canal. Análise similar também é realizada em [28]. Já em [29] é provado, a partir de resultados de canonicidade do DFE apresentados em [30], que a diversidade inerente ao sistema DFE-SC é equivalente à diversidade existente no MFB (do inglês, matched filter bound). Embora os trabalhos citados tratem de sistemas SC, não especificamente com prefixo cíclico, seus resultados podem ser generalizados também para o sistema SCCP.

No contexto com código, em [31] é mostrado que o OFDM consegue atingir a diversidade em frequência de um canal com dois coeficientes Rayleigh. Nesse mesmo artigo é provado que também o sistema DFE-SC consegue atingir a diversidade. Esse artigo considera situações idealizadas em que são utilizados códigos aleatórios associados a entrelaçamento perfeito. Já em [32], é realizada uma análise baseada em simulações na qual é observada que o OFDM consegue explorar a diversidade em frequência do canal, a partir do momento em que um código convolucional é aplicado.

Além da análise das modulações segundo a diversidade frequencial, também vem sendo explorada a comparação no contexto múltiplas entradas e múltiplas saídas (MIMO, do inglês, multiple input multiple output), em que também está presente a diversidade espacial [33] e [34]. Há ainda um terceiro tipo de diversidade: a diversidade temporal. Em canais que variam rapidamente no tempo, é possível explorar tal diversidade aplicando técnicas de entrelaçamento entre símbolos OFDM, ou correspondentes blocos de símbolos SCCP, que tenham sido submetidos a realizações distintas de canais. Apesar do interesse em tais técnicas de diversidade, a análise realizada nessa dissertação considera apenas a diversidade frequencial.

Um fator que merece destaque é que o entrelaçamento não tem apenas a função de explorar diversidade temporal. O entrelaçamento também é fundamental para que o código corretor de erros possa atuar de forma eficaz em canais seletivos em frequência. A forma como os bits são entrelaçados pode interferir de modo considerável nas relações de BER dos sistemas. Embora o entrelaçador tenha uma importância fundamental no desempenho dos sistemas, esse é um fator pouco explorado nos trabalhos de comparação. Grande parte das comparações publicadas, ou não faz referência ao entrelaçador utilizado, ou não indica qualquer critério para a escolha realizada. Diante da falta de trabalhos mais consistentes envolvendo essa questão, é realizada uma detalhada análise no decorrer da dissertação a respeito de múltiplas formas de se realizar o entrelaçamento. 
A dissertação aqui apresentada, portanto, visa oferecer um estudo comparativo entre as técnicas de transmissão, focando naquelas questões que consideramos representar lacunas na literatura de comparação OFDM/SCCP.

\subsection{Contribuições}

As principais contribuições geradas por este trabalho foram:

\section{Transmissão sem codificação de canal}

- Extensão da comparação de desempenho entre os sistemas OFDM e SCCP através de análise da convexidade apresentada em [24] para os casos M-QAM. Essa análise permite constatar a degradação de desempenho do SCCP com o aumento da cardinalidade da modulação.

- Inclusão do equalizador DFE na comparação teórica dos sistemas SCCP e OFDM para modulações M-QAM.

\section{Transmissão com codificação de canal}

- Extensão da análise através da cutoff rate, apresentada em [25], para o SCCP com DFE perfeito.

- Análise através da cutoff rate para modulações M-QAM

- Análise do impacto do projeto do entrelaçador no desempenho dos sistemas, com resultados publicados em [35] e [36].

- Destaque das diferenças entre os casos da análise de canais seletivos em frequência estáticos e canais seletivos em frequência com desvanecimento Rayleigh por blocos.

- Comparação do desempenho dos sistemas OFDM e SCCP, no caso codificado, aplicando o conceito de SNR efetiva.

\subsection{Organização da dissertação}

A dissertação está organizada do seguinte modo:

- Capítulo 2 - Transmissão multiportadora

Nesse capítulo são apresentados os princípios da transmissão multiportadora, a atuação do prefixo cíclico e o modelo universal que será utilizado na comparação dos sistemas. 
- Capítulo 3 - Equalização no domínio da frequência

Nesse capítulo são apresentadas as técnicas de equalização linear e de equalização com decisões realimentadas, mostrando como seus coeficientes ótimos são calculados segundo os critérios MMSE e ZF.

- Capítulo 4 - Efeitos da ordem da modulação

Nesse capítulo os sistemas são comparados, no contexto sem codificação de canal, para diferentes modulações M-QAM. É analisado como a ordem de modulação afeta os sistemas em estudo.

- Capítulo 5 - Sensibilidade em relação à taxa de codificação

Nesse capítulo é considerada uma abordagem de codificação aleatória com blocos de comprimento infinito e os dois sistemas serão comparados em termos de probabilidade de outage por cutoff rate.

- Capítulo 6 - Efeitos do Entrelaçador Nesse capítulo são considerados códigos convolucionais comumente utilizados e é analisado como os sistemas se comportam em termos de BER para os diferentes tipos de entrelaçadores.

- Capítulo 7 - SNR efetiva

Nesse capítulo é aplicado o conceito de SNR efetiva ao sistema OFDM. A partir da aproximação gerada pela SNR efetiva, é apresentada uma nova comparação entre os sistemas OFDM e SCCP.

- Capítulo 8 - Sensibilidade em relação a erros de estimação de canal Neste capítulo, analisamos como se comportam os sistemas em situações em que não há estimação perfeita de canal na recepção.

- Capítulo 9 - Conclusões e Perspectivas 



\section{Capítulo 2}

\section{Transmissão multiportadora}

Neste capítulo serão apresentados os princípios da transmissão multiportadora, sendo dada especial ênfase à questão do prefixo cíclico. Posteriormente, ainda será mostrado que os sistemas de portadora única guardam similaridades com os sistemas multiportadora e que tais semelhanças podem ser traduzidas através de uma abordagem universal.

\subsection{Princípios do sistema OFDM}

Ao longo desse capítulo, considera-se um sistema OFDM com $N$ subportadoras com símbolos complexos formados por pulsos retangulares de duração $T$ segundos. Um possível conjunto ortogonal de subportadoras que pode ser empregado no sistema é o seguinte:

$$
\left.\left\{\phi_{k}(t)\right\}\right|_{k=0} ^{k=N-1}= \begin{cases}e^{j 2 \pi \frac{1}{T} k t} & t \in[0, T] \\ 0 & \text { c.c. }\end{cases}
$$

Note que:

$$
\left\langle\phi_{k}, \phi_{i}\right\rangle=\int_{0}^{T} \phi_{k}(t) \phi_{i}^{*}(t) d t=\int_{0}^{T} e^{j 2 \pi \frac{1}{T}(k-i) t} d t=T \delta(i-k)
$$

Na equação acima, $\delta(i-k)$ indica a função Delta de Kronecker, definida como:

$$
\delta(k)= \begin{cases}1 & k=0 \\ 0 & \text { c.c. }\end{cases}
$$


Então, pode-se modular as subportadoras com símbolos de informação $X_{k}$ e gerar um símbolo OFDM dado por:

$$
x(t)=\sum_{k=0}^{N-1} X_{k} \phi_{k}(t)
$$

Explorando a ortogonalidade do conjunto, um banco de correlatores pode recuperar perfeitamente o bloco de informação $\mathbf{X}=\left[X_{0}, X_{1}, \cdots, X_{N-1}\right]$ :

$$
\left\langle x(t), \phi_{n}(t)\right\rangle=\left\langle\sum_{k=0}^{N-1} X_{k} \phi_{k}(t), \phi_{n}(t)\right\rangle=T X_{n}
$$

A implementação do OFDM no tempo discreto é feita através da amostragem do sinal representado pela eq. (2.3) com período de amostragem $T_{s}=T / N$ :

$$
x_{n}=\left.x(t)\right|_{t=\frac{n T}{N}}=\sum_{k=0}^{N-1} X_{k} e^{j \frac{2 \pi}{N} k n}
$$

Nota-se que o símbolo OFDM pode ser gerado diretamente aplicando a inversa da transformada discreta de Fourier (IDFT) na sequência de dados multiplicado pela constante $N$, o número de subportadoras.

Nesse ponto é válido salientar algumas diferenças existentes entre o símbolo OFDM contínuo e o símbolo OFDM discreto. Em [37] é mostrado que apenas em condições especiais o sinal gerado a partir da conversão D/A do símbolo OFDM discreto é equivalente ao símbolo OFDM gerado diretamente a partir das subportadoras contínuas. Em particular, considerando que formatação retangular de pulsos é empregada, é possível mostrar que apesar dos sinais serem parecidos no tempo, suas características espectrais apresentam diferenças consideráveis. Por tal motivo, como na prática o símbolo OFDM é gerado a partir de sua representação discreta, é preferível estabelecer toda a análise nesse domínio.

No domínio do tempo discreto, o sinal OFDM é expresso como:

$$
x_{n}=\sum_{k=0}^{N-1} X_{k} \Theta_{k, n}
$$

em que $\Theta_{k}(n)$ são as funções de base discretas:

$$
\Theta_{k, n}=e^{j \frac{2 \pi}{N} k n}
$$


com a relação de ortogonalidade sendo descrita por:

$$
\left\langle\Theta_{k, n}, \Theta_{i, n}\right\rangle=\sum_{n=0}^{N-1} \Theta_{k, n} \Theta_{i, n}^{*}=\sum_{n=0}^{N-1} e^{j \frac{2 \pi n}{N}(k-i)}=N \delta(i-k)
$$

Se o canal não introduzir distorções, o símbolo pode ser recuperado a partir da projeção do sinal recebido na direção da subportadora a ele associada:

$$
r_{l}=\left\langle\Theta_{l, n}, x_{n}\right\rangle=\left\langle\Theta_{l, n}, \sum_{k=0}^{N-1} X_{k} \Theta_{k, n}\right\rangle
$$

pela linearidade do produto interno, tem-se:

$$
r_{l}=\sum_{k=0}^{N-1} X_{k}\left\langle\Theta_{k, n}, \Theta_{l, n}\right\rangle=N \sum_{k=0}^{N-1} X_{k} \delta(l-k)=N X_{l}
$$

O problema no uso de tal conjunto ortogonal é observado na situação em que o sinal é submetido a um canal dispersivo no tempo. Nesse caso, a ortogonalidade imposta no transmissor seria perdida no receptor, como visto na seção seguinte. Todavia, felizmente, existe uma técnica que permite manter a ortogonalidade entre as subportadoras nesse caso. Tal técnica é abordada na seção 2.3.

\subsection{Ortogonalidade em canais com multipercurso}

Suponha que a transmissão de um sinal $\mathbf{x}$ de comprimento $N$ se dê num canal seletivo em frequência com resposta impulsiva dada por $\mathbf{h}$. Desconsiderando a atuação do ruído, a sequência de saída é dada por:

$$
\mathbf{y}=\mathbf{h} * \mathbf{x}
$$

em que o símbolo $*$ indica o operador convolução linear:

$$
y_{n}=\sum_{k=0}^{N-1} x_{k} h_{n-k}
$$

Para a recuperação da informação contida na sequência $\mathbf{x}$, é necessário o emprego de técnicas de equalização. Essas técnicas teriam sua complexidade computacional consideravelmente reduzida se a convolução linear indicada na eq. (2.9) fosse uma convolução cíclica, 
i.e.:

$$
\mathbf{y}=\mathbf{h} \circledast \mathbf{x} \leftrightarrow y_{n}=\sum_{k=0}^{N-1} x_{k} h_{n-k \bmod N}
$$

Nessa condições, seria possível aplicar o teorema da convolução da DFT [38] que estabelece que:

$$
\mathbf{y}=\mathbf{h} \circledast \mathbf{x} \leftrightarrow \mathbf{Y}=\mathbf{H} \circ \mathbf{X}
$$

Na expressão acima, $\mathbf{Y}, \mathbf{H}$ e $\mathbf{X}$ correspondem às sequências obtidas a partir da aplicação da transformada de Fourier discreta nas sequências $\mathbf{y}, \mathbf{h}$ e $\mathbf{x}$. O operador $\circ$ representa o produto de Hadamard, ou produto elemento a elemento:

$$
\begin{aligned}
\boldsymbol{a} & =\left[\begin{array}{llll}
a_{1} & a_{2} & \cdots & a_{N}
\end{array}\right]^{T} \\
\boldsymbol{b} & =\left[\begin{array}{llll}
b_{1} & b_{2} & \cdots & b_{N}
\end{array}\right]^{T} \\
\boldsymbol{a} \circ \boldsymbol{b} & =\left[\begin{array}{llll}
a_{1} b_{1} & a_{2} b_{2} & \cdots & a_{N} b_{N}
\end{array}\right]^{T}
\end{aligned}
$$

A expressão do sinal recebido como indicado na eq. (2.12) permite a equalização do sinal através de uma simples correção de ganho e de fase em cada componente de $\mathbf{H}$. Assim, a complexidade do equalizador é consideravelmente reduzida, uma vez que a saída do sistema, no domínio da frequência, passa a ser dada pelo produto termo a termo do símbolo transmitido pela transformada discreta de Fourier do canal.

Uma alternativa para que o sinal recebido possa ser interpretado como a convolução cíclica entre o sinal transmitido e a resposta impulsiva do canal é a adição do prefixo cíclico.

\subsection{Prefixo cíclico}

Uma contribuição importante no desenvolvimento do OFDM ocorreu em 1980, quando Peled e Ruiz propuseram a adição do prefixo cíclico na transmissão [39].

A adição do prefixo cíclico faz com que a convolução linear entre o bloco OFDM transmitido e o canal seja equivalente a uma convolução cíclica, desde que $N_{C P} \geq L-1$, em que $N_{C P}$ é o comprimento do prefixo cíclico e $L$ é o comprimento do canal.

É importante observar que o uso do prefixo cíclico não está restrito à transmissão OFDM. De fato, a extensão cíclica pode ser aplicada a qualquer que seja o bloco a ser transmitido.

Dado um sinal $\mathbf{x}$ a ser transmitido em um canal com resposta impulsiva $\mathbf{h}$, o prefixo 


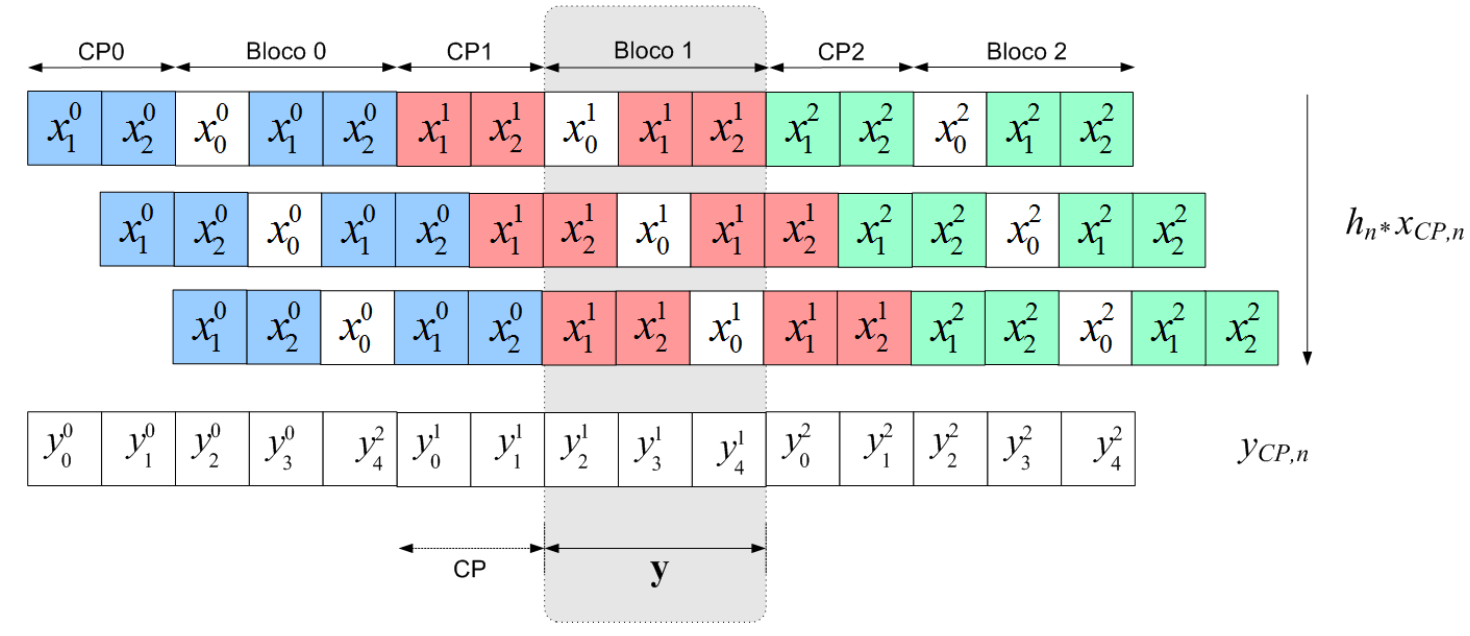

Figura 2.1: Atuação do prefixo cíclico.

cíclico consiste, simplesmente, na transmissão dos últimos elementos do bloco também no início do bloco como mostrado abaixo:

$$
\mathbf{x}_{C P}=\underbrace{\left[x_{N-L+1} \cdots x_{N-1}\right.}_{C P} \quad x_{0} \quad x_{1} \cdots x_{N-1}]
$$

A fim de compreender a atuação do prefixo cíclico, analisemos um exemplo, apresentado na Fig. 2.1, em que o tamanho do bloco é $N=3$, o comprimento do canal é $L=3$ e o tamanho do prefixo cílico é $N_{C P}=2$. A Fig. 2.1 mostra que a janela contendo os elementos $\mathbf{y}=\left\{y_{2}^{1}, y_{3}^{1}, y_{4}^{1}\right\}$ é afetada apenas pelos elementos $\mathbf{x}=\left\{x_{0}^{1}, x_{1}^{1}, x_{2}^{1}\right\}$ da sequência de dados. Mais do que isso: essa janela corresponde à convolução circular de $\mathbf{x}=\left\{x_{0}^{1}, x_{1}^{1}, x_{2}^{1}\right\}$ com o canal.

De modo geral, consideremos a sequência de entrada $\mathbf{x}_{C P} \operatorname{com} N+L-1$ elementos e o canal $\mathbf{h}$ de comprimento $L$. Iremos analisar os elementos $y_{n}$, do vetor de saída $\mathbf{y}$, com $L-1 \leq n<L+N-1$. Denotando essa sequência por $\mathbf{y}$, temos:

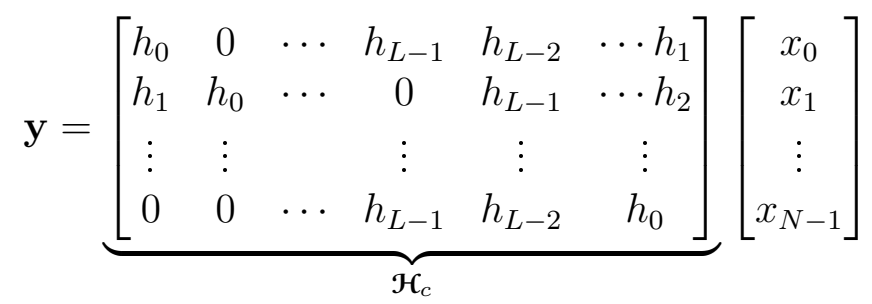

em que a matriz $\mathcal{H}_{c}$ corresponde à matriz de convolução circular do canal com resposta impulsiva $\mathbf{h}$. Em outras palavras, o sinal $\mathbf{y}$ pode ser expresso como $\mathbf{h} \circledast \mathbf{x}$. 
A matriz de convolução do canal apresenta uma estrutura especial que, como será visto a seguir, permite sua diagonalização pela matriz de Fourier. Primeiramente, note que a matriz de convolução circular pode ser reescrita da seguinte forma:

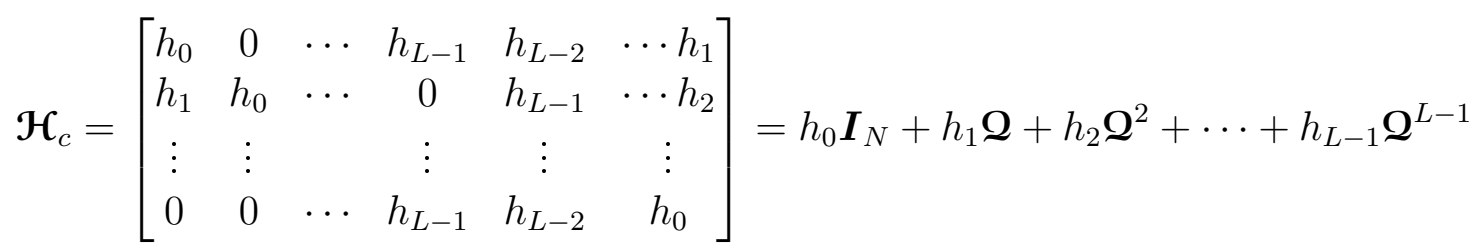

As matrizes $\boldsymbol{I}_{N}$ e $\mathbf{Q}$ correspondem, respectivamente, à matriz identidade de ordem $N$ e à matriz circulante dada por:

$$
\mathbf{Q}=\left[\begin{array}{ccccc}
0 & 0 & \cdots & 0 & 1 \\
1 & 0 & \cdots & 0 & 0 \\
0 & 1 & \cdots & 0 & 0 \\
\vdots & \vdots & & \vdots & \vdots \\
0 & 0 & \cdots & 1 & 0
\end{array}\right]
$$

Utilizando a DFT, pode-se representar a matriz $\mathbf{Q}$ através de uma matriz diagonal $\mathcal{D}$ [40], i.e.,

$$
\mathbf{Q}=\mathcal{F}^{-1} \underbrace{\operatorname{diag}\left\{\exp \left(-j \frac{2 \pi}{N} k\right)\right\}}_{\mathcal{D}} \mathcal{F}=\mathcal{F}^{-1} \mathcal{D F} \quad k=0, \cdots, N-1
$$

O $k$-ésimo elemento da diagonal da matriz $\mathcal{D}$ é dado por $\exp \left(-j \frac{2 \pi}{N} k\right)$ e a matriz $\mathcal{F}$ é a matriz de Fourier, cujos elementos são dados por:

$$
\mathcal{F}_{k, n}=\exp \left(-j \frac{2 \pi}{N}(k-1)(n-1)\right) \quad k, n=1, \cdots, N
$$

e sua inversa tem elementos:

$$
\mathcal{F}_{k, n}^{-1}=\frac{1}{N} \exp \left(j \frac{2 \pi}{N}(k-1)(n-1)\right) \quad k, n=1, \cdots, N
$$

Sobre a matriz de Fourier é ainda importante notar que $\mathcal{F}^{-1}=\frac{1}{N} \mathcal{F}^{H}$, e substituindo (2.17) em (2.15) tem-se:

$$
\mathcal{H}_{C}=h_{0} \boldsymbol{I}_{N}+h_{1} \mathcal{F}^{-1} \mathcal{D} \mathcal{F}+h_{2}\left(\mathcal{F}^{-1} \mathcal{D F}\right)^{2}+\cdots+h_{L-1}\left(\mathcal{F}^{-1} \mathcal{D F}\right)^{L-1}
$$


ou de forma mais compacta:

$$
\mathcal{H}_{C}=\mathcal{F}^{-1}\left(\sum_{n=0}^{L-1} h_{n} \mathcal{D}^{n}\right) \mathcal{F}
$$

Ainda, pela definição da matriz $\mathcal{D}$ indicada na eq. (2.17), a matriz de convolução pode finalmente ser reescrita como:

$$
\begin{aligned}
& \mathcal{H}_{C}=\mathcal{F}^{-1}\left(\sum_{n=0}^{L-1} h_{n} \operatorname{diag}\left\{\exp \left(-j \frac{2 \pi}{N} k\right)\right\}^{n}\right) \mathcal{F} \\
& \mathcal{H}_{C}=\mathcal{F}^{-1} \operatorname{diag}\left\{\sum_{n=0}^{L-1} h_{n} \exp \left(-j \frac{2 \pi}{N} k n\right)\right\} \mathcal{F}
\end{aligned}
$$

Reconhecendo $\sum_{n=0}^{L-1} h_{n} \exp \left(-j \frac{2 \pi}{N} k n\right)$ como $H_{k}$, o $k$-ésimo elemento do vetor transformada discreta de Fourier de $\mathbf{h}$, a expressão (2.20) pode ser simplificada e escrita como:

$$
\mathcal{H}_{C}=\mathcal{F}^{-1} \Lambda \mathcal{F} \quad \boldsymbol{\Lambda}=\operatorname{diag}\{\mathbf{H}\}
$$

A eq. (2.22) mostra que a matriz de convolução circulante do canal é diagonalizável, com elementos diagonais iguais aos elementos da representação em frequência do canal. Além disso, a matriz ortogonal que leva à diagonalização corresponde à própria matriz de Fourier. A estrutura diagonalizável da matriz de convolução circulante do canal faz com que seja possível equalizar o sinal corrigindo a fase e o ganho de cada componente em frequência com apenas um coeficiente. Dá-se o nome à técnica de one-tap equalizer.

O fato é que tanto o prefixo cíclico quanto a idéia de equalização com o one-tap equalizer são aplicáveis a várias técnicas de modulação. A seguir, será visto um arcabouço que permite uma abordagem unificada para tratar diferentes modulações.

\subsection{Abordagem universal}

Como dito na seção 2.1, a implementação discreta do OFDM consiste simplesmente na aplicação da IDFT na sequência de entrada. Desse modo, o modelo da Fig. 2.2 representa o modelo OFDM desde que a matriz $\mathfrak{P}$ seja a própria matriz identidade.

Veja que se a matriz $\mathcal{P}$ for a matriz DFT, a matriz de transmissão equivalente $\mathcal{T}=$ $\mathcal{P F}^{-1}=\mathbf{I}$. Nesse caso, tem-se o sistema SCCP implementado por blocos. 
Desse modo, o mesmo modelo pode ser utilizado para a análise do OFDM e do sistema SCCP. A única diferença entre os dois sistemas reside na matriz de pré-codificação $\mathcal{P}$.

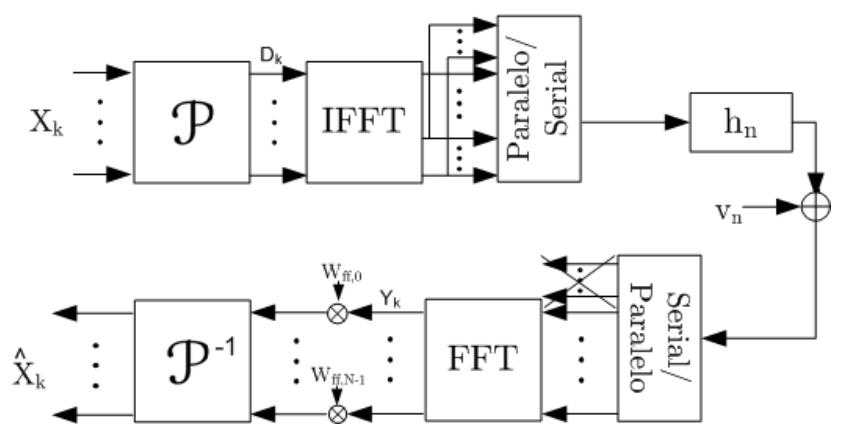

Figura 2.2: Abordagem universal.

A matriz de pré-codificação linear não deve se restringir necessariamente aos casos citados acima. Outros sistemas de transmissão podem ser gerados a partir de mudanças nessa matriz de codificação. Um modelo para o sistema Direct Sequence - Code Division Multiple Access (DS-CDMA), por exemplo, é obtido se as colunas da matriz $\mathcal{P}$ corresponderem aos códigos de espalhamento no domínio da frequência.

É importante ainda ressaltar que o transmissor do caso SCCP tende a ser mais simples do que o do caso OFDM, já que o sinal transmitido não necessita de nenhuma transformação adicional. Por outro lado, a recepção no domínio da frequência é mais custosa, pois é preciso uma IDFT adicional em relação ao OFDM. Fica claro, portanto, que no que diz respeito à complexidade computacional global, os sistemas são equivalentes.

\section{Sumário}

Neste capítulo foram apresentados os princípios de transmissão OFDM, explicando em detalhes a atuação do prefixo cíclico. Também foram mostrados como os conceitos aplicados na transmissão OFDM podem ser refletidos na transmissão SCCP. A ligação entre os dois sistemas foi mais explicitamente indicada na apresentação do modelo universal.

O próximo capítulo será dedicado a técnicas de equalização no domínio da frequência e, através de diferentes critérios, os coeficientes de equalização apresentados no modelo universal serão derivados. 


\section{Capítulo 3}

\section{Equalização no domínio da frequência}

Neste capítulo serão analisadas técnicas de equalização no domínio da frequência. As principais estruturas de equalização serão apresentadas e serão derivadas expressões de relação sinal ruído (SNR, do inglês, signal to noise ratio) a serem utilizadas ao longo da dissertação.

Além da equalização linear, será dada ênfase ao equalizador DFE. Como será detalhado adiante, tal equalizador tem aplicações importantes em canais fortemente seletivos em frequência. Nessas condições, o equalizador linear não provê bons resultados tendo em vista que o erro quadrático médio (MSE, do inglês, mean square error) na saída do equalizador é elevado.

\subsection{Modelo do sistema}

Como é possível inferir a partir das eqs. (2.14) e (2.22), o uso da abordagem universal e do prefixo cíclico permite expressar o sinal recebido como:

$$
\mathbf{y}=\mathcal{H}_{c} \mathcal{F}^{-1} \mathcal{P X}+\boldsymbol{v}
$$

em que $\mathrm{X}$ é a sequência de dados, $\mathcal{H}_{c}$ é a matriz de convolução circular do canal e $\mathcal{F}$ denota a matriz DFT. O vetor $\boldsymbol{v}$ indica o ruído na recepção, que será assumido aditivo gaussiano branco (AWGN, do inglês, additive white Gaussian noise), com média nula e densidade espectral de potência $N_{0} / 2$ por dimensão. 
No domínio da frequência, tem-se:

$$
\mathbf{Y}=\mathcal{F} \mathbf{y}=\underbrace{\mathcal{F H}_{c} \mathcal{F}^{-1}}_{\boldsymbol{\Lambda}} \mathcal{P X}+\underbrace{\mathcal{F} \boldsymbol{v}}_{\Upsilon}
$$

em que a matriz $\Lambda$ é a matriz definida na eq. (2.22).

No caso SCCP, tem-se:

$$
\mathbf{Y}_{S C}=\Lambda \mathcal{F X}+\Upsilon
$$

De modo semelhante, para o caso OFDM, tem-se que a matriz de pré-codificação é a própria matriz identidade de modo que:

$$
\mathbf{Y}_{O F D M}=\Lambda \mathbf{X}+\Upsilon
$$

A ortogonalidade da DFT permite estabelecer um mapeamento direto entre as características estatísticas do vetor aleatório $\boldsymbol{\Upsilon}=\mathcal{F} \boldsymbol{v}$ e do vetor aleatório $\boldsymbol{v}$. Em particular, a aplicação da transformação causa apenas um escalonamento na potência de ruído:

$$
\|\mathcal{F} \boldsymbol{v}\|=\sqrt{(\mathcal{F} \boldsymbol{v})^{H} \cdot \mathcal{F} \boldsymbol{v}}=\sqrt{\boldsymbol{v}^{H} \boldsymbol{N \mathcal { F } ^ { - 1 } \cdot \mathcal { F } \boldsymbol { v }}}=\sqrt{N \boldsymbol{v}^{H} \boldsymbol{v}}=\sqrt{N}\|\boldsymbol{v}\|
$$

Sendo a potência de ruído no domínio do tempo dada por $\sigma_{v}^{2}$, a potência de ruído no domínio da frequência será dada por $\sigma_{\Upsilon}^{2}=N \sigma_{v}^{2}$.

Para o desenvolvimento a seguir, é ainda importante definir a SNR $\gamma$ como:

$$
\gamma=\frac{E\left\{\|\mathcal{P X}\|^{2}\right\}}{E\left\{\|\boldsymbol{\Upsilon}\|^{2}\right\}}
$$

Assumindo que a função autocorrelação da sequência transmitida obedece a: $\mathrm{E}\left\{X_{k} X_{l}^{*}\right\}=$ $\sigma_{X}^{2} \delta(k-l)$, no caso OFDM, $\gamma$ será dada por:

$$
\gamma_{O F D M}=\frac{\sigma_{X}^{2}}{\sigma_{\Upsilon}^{2}}
$$

Já no caso SCCP, a SNR $\gamma$ é dada por:

$$
\gamma_{S C C P}=N \frac{\sigma_{X}^{2}}{\sigma_{\Upsilon}^{2}}=\frac{\sigma_{X}^{2}}{\sigma_{v}^{2}}
$$


É importante notar que o fator de escalonamento $N$, existente na expressão de SNR do sistema SCCP, é resultante do ganho de potência conferido pela matriz de pré-codificação linear $\mathcal{P}$, cuja norma-2 é $N$.

Equalizando o sinal recebido, denotando por $\mathbf{Z}$ o sinal na saída do equalizador, tem-se:

$$
\mathrm{Z}=\mathbf{W} \circ \mathbf{Y}
$$

Note que no caso OFDM, a eq. (3.4) indica que o sinal recebido em cada subportadora é constituído pelo próprio sinal transmitido multiplicado apenas pelo ganho do canal. A fim de equalizá-lo, basta corrigir a fase do sinal. Nos casos de modulação em amplitude, também se faz necessário a correção da magnitude.

Já no caso SCCP, o critério de equalização é crucial, uma vez que a seletividade em frequência do canal gera interferência intersimbólica, a qual precisa ser mitigada através do processo de equalização.

O problema de equalização será tratado de forma segmentada para os dois sistemas em estudo.

\subsection{Sistema OFDM}

Nos sistemas OFDM, a eq. (3.9) se reduz a:

$$
\mathbf{Z}_{O F D M}=\mathbf{W} \circ \mathbf{Y}_{O F D M}=\mathbf{W} \circ(\Lambda \mathbf{X}+\mathbf{\Upsilon})
$$

Como a matriz de pré-codificação OFDM é a própria matriz identidade, o sinal na saída do receptor OFDM é igual à saída do equalizador:

$$
\hat{\mathbf{X}}_{O F D M}=\mathbf{Z}_{O F D M}=\mathbf{W} \circ(\boldsymbol{\Lambda} \mathbf{X}+\mathbf{\Upsilon})
$$

Analisando apenas a $k$-ésima componente do bloco equalizado, tem-se:

$$
\hat{X}_{k}=W_{k} H_{k} X_{k}+W_{k} \Upsilon_{k}
$$

Assim, desde que os coeficientes de equalização corrijam a fase e a magnitude do sinal, a 
SNR na $k$-ésima subportadora é dada por:

$$
\mathrm{SNR}_{k, O F D M}=\mathrm{E}\left\{\left|H_{k}\right|^{2}\right\} \frac{\sigma_{X}^{2}}{\sigma_{\Upsilon}^{2}}=\gamma \mathrm{E}\left\{\left|H_{k}\right|^{2}\right\}
$$

Se a transmissão se der com constelação de módulo constante, uma das possíveis formas de corrigir a fase do sinal é utilizar um filtro casado (MF, do inglês, matched filter). Dessa forma, os coeficientes de equalização são dados simplesmente pelo conjugado complexo da resposta em frequência do canal:

$$
W_{k}=H_{k}^{*}
$$

\subsection{Sistema SCCP}

A abordagem mais simples para equalização é a equalização linear. Neste contexto, utilizando-se o CP é possível implementar tal processo no domínio da frequência através do one-tap equalizer.

A SNR na saída do equalizador linear pode não ser suficiente para que o sistema apresente um desempenho aceitável. Uma alternativa à equalização linear é o equalizador DFE. Tal equalizador é composto por dois filtros, um filtro direto e um filtro de feedback. O filtro direto atenua a ISI pré-cursora e, com a saída do filtro de feedback, é possível cancelar a ISI pós-cursora do sinal recebido.

A seguir serão detalhados os dois processos de equalização, apresentando as expressões de SNR nas saídas dos equalizadores e, em seguida, derivando seus coeficientes ótimos segundo os critérios mínimo erro quadrático médio (MMSE, do inglês, minimum mean square error) e ZF (do inglês, zero forcing).

\subsubsection{Equalização linear}

Com o objetivo de derivar a SNR na saída do equalizador, perceba que de acordo com o esquema na Fig. 2.2, tem-se que:

$$
\mathbf{Z}_{S C}=\mathbf{W} \circ \mathbf{Y}_{S C}=\mathbf{W} \circ(\Lambda \mathcal{F X}+\Upsilon)
$$

Para que a saída do receptor seja obtida, é necessário aplicar a transformação inversa da 
matriz de pré-codificação, i.e., a IDFT:

$$
\hat{\mathbf{X}}_{S C}=\mathcal{F}^{-1}(\mathbf{W} \circ(\boldsymbol{\Lambda F X}+\mathbf{\Upsilon}))
$$

Logo, a estimativa do $k$-ésimo símbolo do bloco pode ser escrita como:

$$
\hat{X}_{k}=\frac{1}{N} \sum_{n=0}^{N-1}\left[W_{n} H_{n} \sum_{m=0}^{N-1} X_{m} e^{-j \frac{2 \pi}{N} n m}\right] e^{j \frac{2 \pi}{N} k n}+\frac{1}{N} \sum_{n=0}^{N-1} W_{n} \Upsilon_{n} e^{j \frac{2 \pi}{N} k n}
$$

em que omitimos o índice $S C$ a fim de simplificar a notação.

Isolando os termos interferentes, tem-se:

$$
\hat{X}_{k}=\underbrace{\frac{1}{N}\left[\sum_{n=0}^{N-1} W_{n} H_{n}\right]}_{\text {signal }} X_{k}+\underbrace{\frac{1}{N} \sum_{\substack{n=0 \\ n \neq k}}^{N-1}\left[\sum_{m=0}^{N-1} W_{m} H_{m} e^{j \frac{2 \pi}{N}(k-m) n}\right] X_{n}}_{\text {interf }}+\underbrace{\frac{1}{N} \sum_{n=0}^{N-1} W_{n} \Upsilon_{n} e^{j \frac{2 \pi}{N} k n}}_{\text {noise }}
$$

Considerando que os termos responsáveis pela interferência inter-simbólica também tem distribuição gaussiana, a SNR na saída do equalizador será dada por:

$$
\mathrm{SNR}=\frac{P_{\text {signal }}}{P_{\text {interf }}+P_{\text {noise }}}
$$

em que $P_{\text {signal }}, P_{\text {interf }}$ e $P_{\text {noise }}$ correspondem às potências do termo referente ao sinal, à interferência inter-simbólica e ao ruído aditivo gaussiano branco, respectivamente. A potência $P_{\text {signal }}$ é expressa como:

$$
P_{\text {signal }}=\mathrm{E}\left\{\left|\frac{1}{N}\left[\sum_{n=0}^{N-1} W_{n} H_{n}\right]\right|^{2}\right\} \sigma_{X}^{2}
$$

Já a potência interferente pode ser calculada partindo da hipótese que a sequência transmitida é i.i.d (independente e identicamente distribuída) e da aplicação da Relação de Parseval [38]:

$$
P_{\text {interf }}=\frac{\sigma_{X}^{2}}{N}\left[\sum_{n=0}^{N-1} \mathrm{E}\left\{\left|W_{n} H_{n}\right|^{2}\right\}-\frac{1}{N} \mathrm{E}\left\{\left|\sum_{n=0}^{N-1} W_{n} H_{n}\right|^{2}\right\}\right]
$$




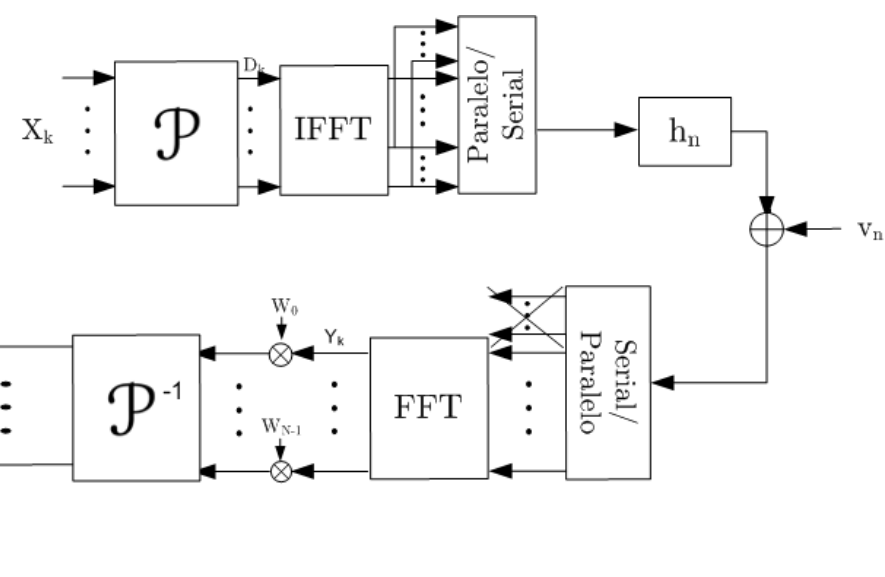

Figura 3.1: Sistema SCCP equalizado com DFE.

Finalmente, a potência de ruído é dada por:

$$
P_{\text {noise }}=\frac{\sigma_{v}^{2}}{N} \sum_{n=0}^{N-1} \mathrm{E}\left\{\left|W_{n}\right|^{2}\right\}
$$

Então, todos os símbolos do bloco têm a mesma SNR, a qual pode ser expressa como:

$$
\mathrm{SNR}=\frac{\frac{1}{N} \mathrm{E}\left\{\left|\left[\sum_{n=0}^{N-1} W_{n} H_{n}\right]\right|^{2}\right\} \sigma_{X}^{2}}{\sigma_{X}^{2}\left[\sum_{n=0}^{N-1} \mathrm{E}\left\{\left|W_{n} H_{n}\right|^{2}\right\}-\frac{1}{N} \mathrm{E}\left\{\left|\sum_{n=0}^{N-1} W_{n} H_{n}\right|^{2}\right\}\right]+\sigma_{v}^{2} \sum_{n=0}^{N-1} \mathrm{E}\left\{\left|W_{n}\right|^{2}\right\}}
$$

\subsubsection{Equalização com decisão realimentada}

O esquema de equalização do sinal SCCP com equalizador com decisões realimentadas é apresentado na Fig. 3.1. Apesar da filtro direto ser implementado no domínio da frequência, o feedback é realizado no domínio do tempo [41].

De acordo com diagrama apresentado na Fig. 3.1, o sinal na saída do equalizador é dado por:

$$
\hat{X}_{k}=z_{f f, k}+\sum_{l=1}^{N_{f b}} w_{f b, l} \hat{X}_{k-l}
$$

em que $z_{f f, k}$ corresponde ao sinal na $k$-ésima componente da saída do filtro direto no domínio do tempo, $w_{f b, l}$ corresponde ao $l$-ésima coeficiente do filtro de feedback e $\hat{X}_{k}$ indica o $k$-ésimo símbolo estimado. Nessa dissertação, considera-se que o filtro de feedback é implementado com $N_{f b}=L-1$ coeficientes, em que $L$ é o comprimento do canal. De acordo com [42] esse 
é o número ótimo de coeficientes de feedback.

Analisando a eq. (3.24), percebe-se uma inconsistência. Note que para a filtragem do primeiro símbolo do bloco, a saída do equalizador DFE dependeria das estimativas obtidas para os símbolos $X_{-1}, X_{-2}, \ldots, X_{-N_{f b}}$. A implementação com prefixo cíclico, indica que tais símbolos correspondem aos últimos símbolos do bloco: $X_{N-1}, X_{N-2}, \ldots, X_{N-N_{f b}}$. O problema é que esses símbolos ainda não foram estimados no momento em que a primeira amostra é processada. Tal problema de causalidade ocorre no processamento dos primeiros $N_{f b}$ símbolos.

A dificuldade de inicialização do equalizador DFE trazida pelo uso do prefixo cíclico pode ser contornada se a abordagem da unique word (UW) for adotada [43]. Nesse caso, a alternativa é substituir o bloco do prefixo cíclico por uma sequência conhecida, a unique word. Dessa forma, os símbolos $X_{-1}, X_{-2}, \ldots, X_{-N_{f b}}$ corresponderiam à UW, a qual é conhecida na recepção. Portanto, tal sequência pode ainda ser interpretada como uma sequência de treinamento. Como pode ser observado na Fig. 3.2, a UW é transmitida antes de cada bloco de informação.

A forma de atuação da UW é idêntica à do CP, e sua inserção faz com que a convolução linear entre a sequência transmitida e a resposta impulsiva do canal possa ser vista como sendo cíclica na janela da FFT, uma vez que os primeiros elementos do bloco transmitido sofrerão interferência dos últimos elementos do bloco, assim como ocorre na transmissão com prefixo cíclico. Em relação ao problema da causalidade encontrado na abordagem do sistema DFE com CP, a UW deve possuir o mesmo comprimento do filtro de feedback. Caso $N_{f b}$ seja menor que $L-1$, pode-se utilizar o CP para que a convolução circular seja obtida.

A Fig. 3.2 mostra como são organizadas as sequências transmitidas nos casos com prefixo cíclico e unique word. A principal diferença entre as duas abordagens diz respeito ao comprimento da janela da FFT utilizada na recepção do sinal. No caso da abordagem com UW, a janela da FFT deve compreender os blocos de dados e o bloco da UW. Já no caso de transmissão com CP, a FFT é realizada apenas no bloco de dados. Portanto, para manter o mesmo comprimento da FFT nas duas técnicas, teríamos que transmitir menos dados por bloco se a transmissão por UW fosse adotada. Entretanto, é importante observar que a UW pode ser usada para sincronismo e estimação de canal. Já na transmissão com CP é necessário alocar algumas subportadoras para sinais piloto a fim de realizar tais tarefas. A partir desse ponto de vista, as duas abordagens tendem a ser equivalentes.

Apesar dos problemas gerados na inicialização do filtro de feedback, a abordagem com CP será adotada no caso do DFE, de modo a simplificar a comparação entre os sistemas, pois as estruturas serão, assim, equivalentes. Nas simulações, o feedback será corretamente inicializado com os últimos símbolos transmitidos. 


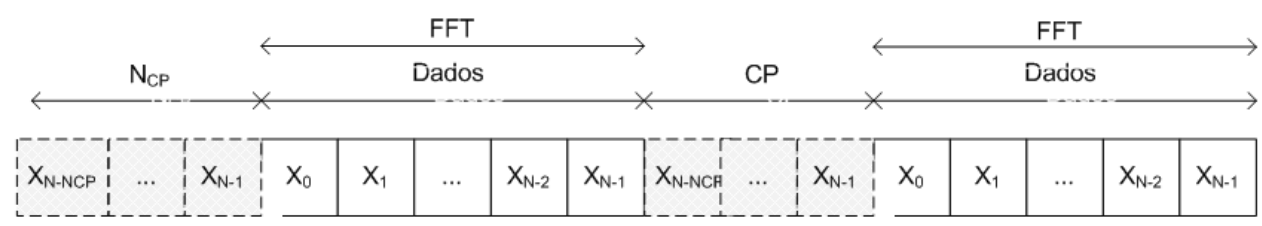

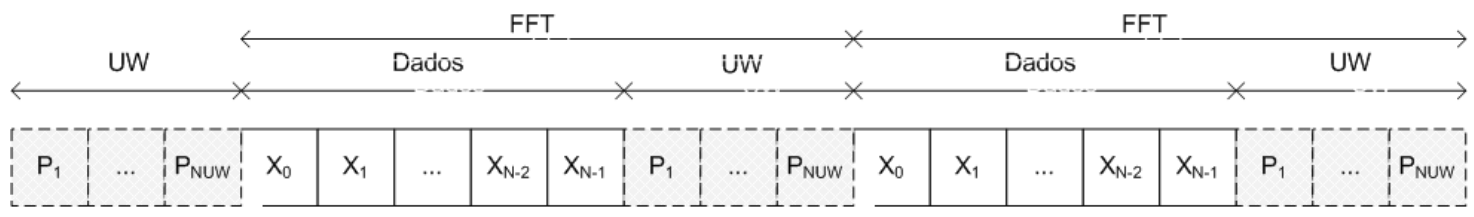

Figura 3.2: Estrutura da sequência para os casos com CP e UW.

A principal desvantagem no uso do equalizador DFE é sua sensibilidade à propagação de erros. Caso haja erros no processo de decisão de símbolos, tais símbolos errados irão ser realimentados no filtro de feedback e contribuirão para um aumento do MSE. Nessas condições, se codificação de canal for empregada, o desempenho do equalizador DFE pode ser inferior ao desempenho do equalizador linear. Esse fato é discutido em [44] em que é apresentada uma análise, no contexto de SC-FDMA (do inglês, Single Carrier Frequency Division Multiple Access). Nesse trabalho é mostrado que, apesar do DFE apresentar menos blocos com erros do que o sistema equalizado com filtro linear, a propagação de erros faz com que a probabilidade de que ocorram blocos com mais erros em rajada do que a capacidade de correção do código é maior no DFE do que no LE.

Alguns trabalhos foram desenvolvidos com o objetivo de modelar o fenômeno de propagação de erros no DFE e.g. [45], [46], [47]. A maioria dos modelos gerados partem da premissa que a propagação de erros pode ser modelada por uma cadeia de Markov, chegando a resultados complexos e de difícil manipulação. Tais análises fogem do escopo do nosso trabalho. Portanto em todas as análises teóricas desenvolvidas ao longo do trabalho, será assumido que decisões corretas serão realimentadas no filtro de feedback. Essa idealização do DFE livre de propagação erros será denotada por DFE perfeito.

Na seção seguinte serão determinados os coeficientes do equalizador. Serão abordados os dois critérios de equalização mais difundidos: o ZF e o MMSE. 


\subsection{Critérios de equalização}

\subsubsection{Critério zero forcing}

O critério ZF consiste em eliminar a ISI sem levar em consideração a potência de ruído. Os coeficientes de equalização que satisfazem tal condição são aqueles que invertem o canal:

$$
W_{Z F, k}=\frac{1}{H_{k}}
$$

Aplicando os coeficientes nas expressões de SNR dadas pelas eq. (3.23):

$$
\mathrm{SNR}_{Z F}^{L E S C}=\frac{1}{\frac{1}{N} \sum_{k=0}^{N-1} \frac{1}{\gamma\left|H_{k}\right|^{2}}}
$$

Pode-se ainda escrever a eq.(3.26) em função do operador média harmônica:

$$
\operatorname{SNR}_{Z F}^{L E S C}=\text { harmmean }\left\{\gamma|\mathbf{H}|^{2}\right\}
$$

em que para uma sequência qualquer $\mathbf{x}$ com $N$ elementos tem-se:

$$
\text { harmmean }\{\mathbf{x}\}=\frac{1}{\frac{1}{N} \sum_{k=0}^{N-1} x_{k}}
$$

Note que se alguma subportadora for fortemente desvanecida, há uma grande amplificação do ruído. Esse é o principal problema do equalizador ZF. Uma alternativa a esse problema é dada pelo equalizador MMSE.

\subsubsection{Critério do mínimo erro quadrático médio}

Como alternativa ao critério de equalização ZF, o critério MMSE fornece uma solução impondo um compromisso entre eliminação da ISI e minimização dos efeitos de amplificação do ruído. Desse modo, é contornado o principal problema do critério ZF.

O equalizador MMSE apenas inverte o canal em situações em que a SNR é muito elevada. Assim, após a equalização, é natural que haja um nível residual de ISI. 


\section{Filtro Linear}

A fim de derivar os coeficientes MMSE, deve-se ter em mente que, no caso de filtragem linear, o sinal recebido pelo equalizador é da seguinte forma:

$$
\mathbf{Y}=\Lambda \mathcal{P X}+\Upsilon
$$

Por simplicidade de notação, seja $\mathbf{D}=\mathfrak{P X}$ o vetor de sinais desejados no domínio da frequência. Denotando o vetor de coeficientes do equalizador por $\mathbf{W}$, o critério de equalização MMSE é dado pela seguinte expressão:

$$
\underset{\mathbf{W}}{\operatorname{argmin}} \mathrm{E}\left\{\|\mathbf{D}-\mathbf{W} \circ \mathbf{Y}\|^{2}\right\}
$$

Analisando a função custo, tem-se:

$$
\begin{aligned}
J & =\mathrm{E}\left\{\|\mathbf{D}-\mathbf{W} \circ \mathbf{Y}\|^{2}\right\} \\
& =\mathbf{E}\left\{\mathbf{D}^{H} \mathbf{D}+(\mathbf{W} \circ \mathbf{Y})^{H}(\mathbf{W} \circ \mathbf{Y})-(\mathbf{W} \circ \mathbf{Y})^{H} \mathbf{D}-\mathbf{D}^{H}(\mathbf{W} \circ \mathbf{Y})\right\}
\end{aligned}
$$

Com o objetivo de minimizar a função custo, é necessário derivá-la em relação aos coeficientes do filtro e a igualar a zero. Para tanto, pode-se aplicar os conceitos do cálculo de Wirtinger [48] e derivar a função em relação ao conjugado da variável considerando $W_{k}$ constante. Desse modo, pode-se diferenciar a função custo aplicando as mesmas regras válidas para diferenciação em relação a variáveis reais.

$$
\frac{\partial}{\partial W_{k}^{*}} J=\mathrm{E}\left\{Y_{k}^{*} Y_{k} W_{k}-D_{k} Y_{k}^{*}\right\}
$$

Impondo a condição de derivada nula:

$$
W_{k}=\frac{\mathrm{E}\left\{D_{k} Y_{k}^{*}\right\}}{\mathrm{E}\left\{Y_{k}^{*} Y_{k}\right\}}=\frac{\mathrm{E}\left\{D_{k}\left(D_{k} H_{k}+\Upsilon_{k}\right)^{*}\right\}}{\mathrm{E}\left\{\left|D_{k} H_{k}+\Upsilon_{k}\right|^{2}\right\}}
$$

Logo, os coeficientes do equalizador são expressos por:

$$
W_{M M S E, k}=\frac{H_{k}^{*}}{\left|H_{k}\right|^{2}+\frac{\sigma_{\Upsilon}^{2}}{\sigma_{D}^{2}}}
$$


Aplicando os coeficiente encontrados na eq.(3.23), tem-se que a SNR na saída do equalizador linear MMSE é dada por:

$$
\mathrm{SNR}_{M M S E}^{L E S C}=\frac{\frac{1}{N} \sum_{k=0}^{N-1} \frac{\sigma_{D}^{2}\left|H_{k}\right|^{2}}{\sigma_{D}^{2}\left|H_{k}\right|^{2}+\sigma_{\Upsilon}^{2}}}{\frac{1}{N} \sum_{k=0}^{N-1} \frac{\sigma_{\Upsilon}^{2}}{\sigma_{D}^{2}\left|H_{k}\right|^{2}+\sigma_{\Upsilon}^{2}}}
$$

que pode ainda ser escrita como:

$$
\mathrm{SNR}_{M M S E}=\frac{1}{\frac{1}{N} \sum_{k=0}^{N-1} \frac{1}{1+\gamma\left|H_{k}\right|^{2}}}-1
$$

Para simplificar a notação, suprimimos o índice LESC da expressão acima.

Como será visto, é conveniente expressar a eq.(3.35) em termos da média harmônica:

$$
\operatorname{SNR}_{M M S E}=\text { harmmean }\left(1+\gamma|\mathbf{H}|^{2}\right)-1
$$

Comparando as eqs. (3.26) e (3.35), nota-se que se alguma subportadora for fortemente desvanecida, a SNR na saída do equalizado ZF tende a zero, independentemente das outras subportadoras, fato que não é observado no equalizador MMSE. Portanto, o equalizador MMSE parece apresentar um desempenho melhor do que o equalizador ZF. A seguir será demonstrado que, de fato, $\mathrm{SNR}_{M M S E} \geq \mathrm{SNR}_{Z F}$ e, para tanto, será desenvolvida uma prova baseada em características de convexidade de funções.

Esse será um artifício exaustivamente utilizado ao longo de todo esse trabalho. O apêndice A mostra um teorema que fundamenta muitas das demonstrações desenvolvidas neste trabalho e indica algumas provas de desigualdades clássicas explorando características de convexidade de funções.

Pela eq.(3.26), pode-se definir $g(x)=\frac{1}{x}$ e reescrever a $\mathrm{SNR}_{Z F}$ da seguinte forma:

$$
\mathrm{SNR}_{Z F}=g^{-1}\left(\frac{1}{N} \sum_{k=0}^{N-1} g\left(\gamma\left|H_{k}\right|^{2}\right)\right)
$$

Pode-se ainda definir $f(x)=\frac{1}{1+x}$, com inversa $f^{-1}(x)=\frac{1}{x}-1$. Então, é possível reescrever 
SNR $_{M M S E}$ dada na eq.(3.35) como:

$$
\mathrm{SNR}_{M M S E}=f^{-1}\left(\frac{1}{N} \sum_{k=0}^{N-1} f\left(\gamma\left|H_{k}\right|^{2}\right)\right)
$$

Aplicando a função $f(x)$ nas eqs.(3.37) e (3.38), tem-se:

$$
\begin{gathered}
f\left(\mathrm{SNR}_{Z F}\right)=f\left(g^{-1}\left(\frac{1}{N} \sum_{k=0}^{N-1} g\left(\gamma\left|H_{k}\right|^{2}\right)\right)\right. \\
f\left(\mathrm{SNR}_{M M S E}\right)=\frac{1}{N} \sum_{k=0}^{N-1} f\left(\gamma\left|H_{k}\right|^{2}\right)
\end{gathered}
$$

Definindo $t(x)=f\left(g^{-1}(x)\right)$, pode-se escrever:

$$
\begin{gathered}
f\left(\mathrm{SNR}_{Z F}\right)=t\left(\frac{1}{N} \sum_{k=0}^{N-1} g\left(\gamma\left|H_{k}\right|^{2}\right)\right) \\
f\left(\mathrm{SNR}_{M M S E}\right)=\frac{1}{N} \sum_{k=0}^{N-1} t\left(g\left(\gamma\left|H_{k}\right|^{2}\right)\right)
\end{gathered}
$$

Mas $t(x)=\frac{x}{1-x}$ é côncava, já que sua segunda derivada é negativa:

$$
\frac{d^{2}}{d x^{2}} t(x)=-\frac{2}{(1+x)^{2}} \leq 0
$$

A Desigualdade de Jensen [49] afirma que se uma função $t(x)$ for côncava, a seguinte desigualdade é válida:

$$
\frac{1}{N} \sum_{k=0}^{N-1} t\left(x_{k}\right) \leq t\left(\frac{1}{N} \sum_{k=0}^{N-1} x_{k}\right)
$$

quaisquer que sejam os elementos $x_{k}$ pertencentes ao domínio de $t(x)$.

Aplicando tal desigualdade, concluímos que $f\left(\mathrm{SNR}_{M M S E}\right) \leq f\left(\mathrm{SNR}_{Z F}\right)$. Lembrando que $f(x)$ é uma função decrescente, concluímos que $\operatorname{SNR}_{M M S E} \geq \mathrm{SNR}_{Z F}$. Tal relação é também demonstrada em [50].

Então, nos sistemas SCCP, o desempenho do equalizador MMSE é de fato superior ao desempenho do equalizador ZF, desde que a hipótese sobre a gaussianidade da ISI seja válida.

Por esse motivo, ao longo de toda a dissertação, será considerado que o critério MMSE 
será empregado na equalização dos sistemas SCCP.

\section{DFE}

De acordo com a Fig. 3.1, no domínio da frequência, o sinal na entrada do decisor é dado por:

$$
\mathbf{Z}_{D F E}=\mathbf{Z}_{F F}+\mathbf{Z}_{F B}
$$

em que $\mathbf{Z}_{F F}$ corresponde à saída do filtro direto e $\mathbf{Z}_{F B}$ corresponde à saída do filtro de feedback.

Mas, $\mathbf{Z}_{F F}=\mathbf{Y} \circ \mathbf{W}_{f f}=(\boldsymbol{\Lambda} \mathbf{D}+\mathbf{\Upsilon}) \circ \mathbf{W}_{f f}$ e, considerando que decisões corretas são realimentadas no DFE, $\mathbf{Z}_{F B}=\mathbf{W}_{f b} \circ \mathbf{D}$.

Dessa forma:

$$
\mathbf{Z}_{D F E}=(\Lambda \mathbf{D}+\Upsilon \mathbf{\Upsilon}) \circ \mathbf{W}_{f f}+\mathbf{W}_{f b} \circ \mathbf{D}
$$

A função custo correspondente pode ser expressa como:

$$
J_{D F E}=\mathrm{E}\left\{\left\|\mathbf{D}-(\Lambda \mathbf{D}+\mathbf{\Upsilon}) \circ \mathbf{W}_{f f}+\mathbf{W}_{f b} \circ \mathbf{D}\right\|\right\}
$$

ou ainda:

$$
J_{D F E}=\sum_{k=0}^{N-1} \sigma_{\Upsilon}^{2}\left|W_{f f, k}\right|^{2}+\sigma_{D}^{2}\left|1-\left(W_{f f, k} H_{k}+W_{f b, k}\right)\right|^{2}
$$

Fixando os coeficientes do filtro de feedback, pode-se calcular os coeficientes do filtro direto a partir do gradiente de (3.48) em relação aos coeficientes do filtro direto $W_{f f, k}$.

Procedendo de modo similar ao realizado no caso da filtragem linear, os coeficientes do filtro direto que minimizam a $J_{D F E}$ são dados por:

$$
W_{f f, k}=\frac{\sigma_{D}^{2} H_{k}^{*}\left(1-W_{f b, k}\right)}{\sigma_{D}^{2}\left|H_{k}\right|^{2}+\sigma_{\Upsilon}^{2}}
$$

Substituindo tais coeficientes na expressão da função custo indicada na eq.(3.48) e lembrando que $\frac{\sigma_{D}^{2}}{\sigma_{\Upsilon}^{2}}=\gamma$, tem-se:

$$
J=\frac{\sigma_{\Upsilon}^{2}}{N} \sum_{k=0}^{N-1} \frac{\left|1-W_{f b, k}\right|^{2}}{\left|H_{k}\right|^{2}+\gamma}
$$


Expressando os coeficientes do filtro de feedback no domínio do tempo:

$$
J=\frac{\sigma_{\Upsilon}^{2}}{N} \sum_{k=0}^{N-1} \frac{1}{\left|H_{k}\right|^{2}+\gamma}\left|1-\sum_{n=0}^{N_{f b}-1} w_{f b, n} e^{-j \frac{2 \pi}{N} n k}\right|^{2}
$$

Aplicando os conceitos do cálculo de Wirtinger novamente, deriva-se a função custo em relação ao conjugado do coeficiente de feedback para obter seu gradiente. A $m$-ésima componente do gradiente tem a seguinte forma:

$$
\nabla_{m} J=-\frac{1}{N} \sum_{k=0}^{N-1} \frac{1}{\left|H_{k}\right|^{2}+\gamma} e^{j 2 \pi \frac{k m}{N}}\left(1-\sum_{n=0}^{N-1} w_{f b, n} e^{-j \frac{2 \pi}{N} n k}\right)
$$

Portanto, a condição de gradiente nulo é satisfeita para a solução do seguinte sistema de equações:

$$
\mathcal{B} \mathbf{w}_{\mathrm{fb}}=\mathbf{b}
$$

em que:

$$
\mathcal{B}_{m, n}=\sum_{k=0}^{N-1} \frac{e^{-j 2 \pi \frac{k(n-m)}{N}}}{\left|H_{k}\right|^{2}+\gamma} \quad \mathbf{b}_{m}=\sum_{k=0}^{N-1} \frac{e^{j 2 \pi \frac{k m}{N}}}{\left|H_{k}\right|^{2}+\gamma}
$$

Maiores detalhes a respeito da implementação do equalizador DFE no domínio da frequência podem ser encontrados em [20] e [41].

Finalmente, ainda é possível calcular a SNR na saída do equalizador dado que os coeficientes do DFE são definidos de acordo com as eqs.(3.49) e (3.53). Em [51], é mostrado que a MMSE do DFE perfeito pode ser expressa como:

$$
J_{m i n, D F E}=\exp \left\{\frac{1}{N} \sum_{k=0}^{N-1} \log \left(\frac{1}{1+\gamma\left|H_{k}\right|^{2}}\right)\right\}
$$

Mas de acordo com [52], a SNR se relaciona com a MMSE como se segue:

$$
\mathrm{SNR}=\frac{1}{J_{\min }}-1
$$


Logo:

$$
\begin{aligned}
\mathrm{SNR}_{D F E}=\frac{1}{J_{\min , D F E}}-1 & =\exp \left\{\frac{1}{N} \sum_{k=0}^{N-1} \log \left(1+\gamma\left|H_{k}\right|^{2}\right)\right\}-1 \\
& =\prod_{k=0}^{N-1}\left(1+\gamma\left|H_{k}\right|^{2}\right)^{\frac{1}{N}}-1 \\
& =\text { geomean }\left\{1+\gamma\left|H_{k}\right|^{2}\right\}-1
\end{aligned}
$$

O operador geomean $\{\cdot\}$ indica a média geométrica. Dado uma sequência de números reais positivos, x, a média geométrica é definida como:

$$
\text { geomean }\{\mathbf{x}\}=\prod_{k=0}^{N-1} x_{k}^{\frac{1}{N}}
$$

Nesse ponto, pode-se utilizar o fato, provado no apêndice A, de que a média geométrica de um conjunto não-negativo é sempre maior que a média harmônica e comparar as eqs.(3.36) e (3.59). Portanto, como já esperado, concluímos que a SNR do DFE perfeito é sempre maior que a SNR do filtro linear.

Ainda é válido destacar que de acordo com o desenvolvimento em [51], a expressão de SNR dada em (3.59) é válida na situação em que o filtro direto é implementado com infinitos coeficientes. O uso do CP faz com que seja possível atingir esse valor de SNR com um número finito de coeficientes.

\section{Sumário}

Neste capítulo foram discutidas as estruturas de equalização no domínio da frequência e as expressões de SNR obtidas na saída do equalizador nos contextos apresentados.

Mostrou-se que o critério de equalização MMSE sempre leva a uma SNR maior que o critério ZF. Por esse motivo, definimos o MMSE como critério de equalização adotado ao longo da dissertação.

Além da equalização linear, o equalizador DFE foi apresentado e foi demonstrado que o DFE perfeito sempre leva a uma SNR maior do que a do equalizador linear.

Ainda foi apresentado um método de demonstração de desigualdades, fundamentado em características de convexidades de funções, que será utilizado por toda a dissertação.

No próximo capítulo, será analisado como a SNR pode ser mapeada no contexto de comparação em termos de BER. 



\section{Capítulo 4}

\section{Efeitos da ordem da modulação}

Neste capítulo, será verificado como a cardinalidade da modulação interfere nas relações de BER entre os sistemas estudados. Ao longo deste capítulo, com o objetivo de facilitar o tratamento analítico, ainda será dado destaque ao contexto de transmissão sem codificação de canal. Apesar da codificação de canal afetar diferentemente os sistemas OFDM e SCCP, será visto no capítulo seguinte que muitas das conclusões obtidas na situação não-codificada são refletidas em sistemas que fazem uso de codificação de canal.

Para verificar os efeitos da ordem da modulação, será estabelecida uma comparação de desempenho, envolvendo os sistemas SCCP e OFDM, fundamentada em relações de convexidade das expressões obtidas para BER. A ideia para essa abordagem surgiu na comparação feita em [24], em que era apresentada uma comparação de BER restrita às constelações QPSK e 16-QAM e a equalização linear. Fundamentados no teorema indicado no apêndice 1, estenderemos a análise às constelações 64-QAM e 256-QAM e ao SCCP equalizado com DFE.

\subsection{Derivação de expressões de BER}

De acordo com [52], se uma constelação M-QAM for utilizada na modulação dos bits, para uma dada SNR, a probabilidade de erro de símbolo (SER, do inglês, symbol error probability) é dada por:

$$
\mathrm{SER}=4\left(1-\frac{1}{\sqrt{M}}\right) Q\left(\sqrt{\frac{3}{M-1} \mathrm{SNR}}\right)\left[1-\left(1-\frac{1}{\sqrt{M}}\right) Q\left(\sqrt{\frac{3}{M-1} \mathrm{SNR}}\right)\right]
$$

Da mesma forma do capítulo 3, a expressão para probabilidade de erro dada por 4.1 é válida desde que seja assumido um modelo gaussiano para a ISI. 
Ainda é possível aproximar a eq. (4.1) por:

$$
\mathrm{SER} \approx 4\left(1-\frac{1}{\sqrt{M}}\right) Q\left(\sqrt{\frac{3}{M-1} \mathrm{SNR}}\right)
$$

Se for empregado mapeamento de Gray, é razoável supor que, para valores médios ou altos de SNR, em cada erro de símbolo há apenas um erro de bit, de modo que a expressão para probabilidade de erro de bit em cada subportadora pode ser aproximada por:

$$
\mathrm{BER} \approx \frac{4}{\log _{2}(M)}\left(1-\frac{1}{\sqrt{M}}\right) Q\left(\sqrt{\frac{3}{M-1} \mathrm{SNR}}\right)
$$

Definindo:

$$
\begin{gathered}
\alpha=\frac{4}{\log _{2}(M)}\left(1-\frac{1}{\sqrt{M}}\right) \\
\beta=\frac{3}{M-1}
\end{gathered}
$$

pode-se reescrever a eq. (4.3) como:

$$
\mathrm{BER} \approx \alpha Q(\sqrt{\beta \mathrm{SNR}})
$$

\subsubsection{BER no sistema OFDM}

No caso do sistema OFDM, a SNR em cada subportadora é dada pela eq.(3.13). Por conveniência, a equação é aqui reproduzida:

$$
\mathrm{SNR}_{k}=\gamma\left|H_{k}\right|^{2}
$$

Como, no contexto sem codificação de canal, os sinais nas subportadoras são independentes, a BER do sistema OFDM será dada pela média da BER de todas as subportadoras:

$$
\mathrm{BER}_{O F D M}=\frac{1}{N} \sum_{k=0}^{N-1} \alpha Q\left(\sqrt{\beta \gamma\left|H_{k}\right|^{2}}\right)
$$




\subsubsection{BER no sistema SCCP}

No sistema SCCP, os símbolos associados a um mesmo bloco têm a mesma SNR. Para diferentes tipos de equalizadores, as expressões de SNR foram apresentadas no capítulo anterior. Como já demonstrado anteriormente, o critério MMSE é superior ao ZF, por tal motivo, a análise realizada nesse capítulo será restrita ao critério MMSE.

No caso de equalização linear, deduzimos que a SNR na saída do equalizador é dada por:

$$
\operatorname{SNR}_{L E}=\text { harmmean }\left(1+\gamma|\mathbf{H}|^{2}\right)-1
$$

Já que não será tratado outro critério de equalização, o índice MMSE encontrado na eq.(3.36) será suprimido. Além disso, para diferenciar da SNR na saída do equalizador DFE, o termo LE será inserido. Logo, pela eq.(4.6), a BER do SCCP equalizado com filtro linear pode ser expressa por:

$$
\mathrm{BER}_{L E}=\alpha Q\left(\sqrt{\beta \mathrm{SNR}_{L E}}\right)=\alpha Q\left(\sqrt{\beta\left(\text { harmmean }\left(1+\gamma|\mathbf{H}|^{\mathbf{2}}\right)-1\right)}\right)
$$

Em relação ao DFE, tem-se que a SNR na saída do equalizador pode ser escrita como:

$$
\operatorname{SNR}_{D F E}=\text { geomean }\left\{1+\gamma\left|H_{k}\right|^{2}\right\}-1
$$

Portanto, a BER do sistema DFE-SCCP perfeito pode ser escrita como:

$$
\mathrm{BER}_{D F E}=\alpha Q\left(\sqrt{\beta \mathrm{SNR}_{D F E}}\right)=\alpha Q\left(\sqrt{\beta\left(\text { geomean }\left(1+\gamma|\mathbf{H}|^{2}\right)-1\right)}\right)
$$

\subsection{Comparação de desempenho}

$\mathrm{Na}$ seção anterior, foram apresentadas as expressões de BER para o OFDM e para o SCCP. Nesta seção, prosseguiremos com uma análise de convexidade dessas funções de probabilidade e determinaremos em quais condições de canal uma técnica se sobressai em relação a outra.

De acordo com o exposto na capítulo anterior, sabe-se que a SNR do filtro linear é sempre inferior à SNR na saída do DFE perfeito. Sendo a função de BER estritamente decrescente com a SNR, conclui-se que a BER apresentada pelo DFE será sempre inferior à BER apresentada pelo SCCP equalizado com filtro linear. Essa conclusão é válida desde que a hipótese sobre a gaussianidade da SNR seja, de fato, adequada. 
Em relação ao OFDM, a comparação não é tão imediata assim, já que a expressão de BER dada por eq.(4.8) é estruturalmente diferente das expressões em (4.10) e (4.12).

Primeiramente, será feita a comparação entre os sistemas OFDM e LE-SCCP. Em seguida, a relação entre os sistemas OFDM e DFE-SCCP será tratada.

\subsubsection{OFDM vs LE-SCCP}

A fim de comparar a probabilidade de erro do sistema OFDM com a do sistema LE-SCCP define-se a seguinte função auxiliar:

$$
h(x)=\alpha Q\left(\sqrt{\beta \frac{1-x}{x}}\right) \Leftrightarrow \alpha Q(\sqrt{\beta x})=h\left(\frac{1}{1+x}\right), \quad 0 \leq x \leq 1
$$

Pode-se perceber que as expressões para a BER do OFDM, apresentada na eq. (4.8), e do sistema SCCP, descrita na eq. (4.10), podem ser reescritas em termos da função $h(x)$. Dessa forma:

$$
\mathrm{BER}_{O F D M}=\frac{1}{N} \sum_{k=0}^{N-1} \alpha Q\left(\sqrt{\beta \gamma\left|H_{k}\right|^{2}}\right) \Leftrightarrow \operatorname{BER}_{O F D M}=\frac{1}{N} \sum_{k=0}^{N-1} h\left(\frac{1}{1+\gamma\left|H_{k}\right|^{2}}\right)
$$

$\mathrm{BER}_{L E}=\alpha Q\left(\sqrt{\beta\left(\text { harmmean }\left(1+\gamma|\mathbf{H}|^{2}\right)-1\right)}\right) \Leftrightarrow B E R_{L E}=h\left(\frac{1}{N} \sum_{k=0}^{N-1} \frac{1}{1+\gamma\left|H_{k}\right|^{2}}\right)$

Pode-se definir ainda o seguinte mapeamento:

$$
x_{k}=\frac{1}{1+\gamma\left|H_{k}\right|^{2}}
$$

Nessas condições:

$$
\begin{gathered}
\mathrm{BER}_{O F D M}=\frac{1}{N} \sum_{k=0}^{N-1} h\left(x_{k}\right) \\
\mathrm{BER}_{L E}=h\left(\frac{1}{N} \sum_{k=0}^{N-1} x_{k}\right)
\end{gathered}
$$

Da mesma forma que detalhado no teorema 1 do apêndice A, as características de con- 
vexidade da função $h(x)$ serão exploradas para relacionar a $\mathrm{BER}_{O F D M}$ com a $\mathrm{BER}_{L E}$. Para tanto, é necessário analisar a segunda derivada da função $h(x)$. A primeira derivada de $h(x)$ é dada por:

$$
\frac{d}{d x} h(x)=\frac{d}{d x} \alpha Q\left(\sqrt{\beta \frac{1-x}{x}}\right)=-\alpha \frac{1}{2 x^{2}} \sqrt{\frac{\beta x}{1-x}} \frac{1}{\sqrt{2 \pi}} \exp \left(-\frac{\beta}{2}\left(\frac{1}{x}-1\right)\right)
$$

da qual obtemos então a segunda derivada:

$$
\frac{d^{2}}{d x^{2}} h(x)=\frac{1}{x} \frac{d}{d x} h(x) \underbrace{\left(-2+\frac{\beta}{2 x}+\frac{1}{2(1-x)}\right)}_{a(x)}
$$

Sabendo que $h(x)$ é uma função crescente, sua primeira derivada é estritamente positiva. Desse modo, o sinal de $\frac{d^{2}}{d x^{2}} h(x)$ será determinado pelo sinal da função $a(x)$.

No caso QPSK, $\beta=1$. Assim, a função $a(x)$ assume a seguinte forma:

$$
a(x)=-2+\frac{1}{2 x(1-x)}
$$

Observe que minimizar $a(x)$ equivale a maximizar $x(1-x)$. Dessa forma, o valor mínimo de $a(x)$ é atingido para $x_{\text {min }}=1 / 2 \operatorname{com} a\left(x_{\text {min }}\right)=0$. Portanto, a função $a(x)$ é não-negativa, fato que torna $\frac{d^{2}}{d x^{2}} h(x) \geq 0$, ou equivalentemente, torna $h(x)$ uma função convexa. A Fig. 4.1 mostra a função $h(x)$ e sua segunda derivada. É importante destacar que o intervalo de domínio da função $h(x)$ corresponde a $x \in[0,1]$, já que as eqs.(4.14), (4.15) e (4.16) indicam que $h(x)$ é calculada nos pontos $0<x_{k} \leq 1$.

A convexidade da função $h(x)$ garante:

$$
h\left(\frac{1}{N} \sum_{k=0}^{N-1}\left(\frac{1}{1+\gamma\left|H_{k}\right|^{2}}\right)\right) \leq \frac{1}{N} \sum_{k=0}^{N-1} h\left(\frac{1}{1+\gamma\left|H_{k}\right|^{2}}\right)
$$

qualquer que seja a condição de canal.

Em outras palavras, $B_{L E} \leq \mathrm{BER}_{O F D M}$ para modulação QPSK.

Generalizando para outras modulações M-QAM, a função $a(x)$ assume a seguinte forma:

$$
a(x)=-2+\frac{1}{2}\left(\frac{\beta}{x}+\frac{1}{1-x}\right)
$$

Vejamos para quais valores de $x$, a função $a(x)$, acima representada, assume valores 

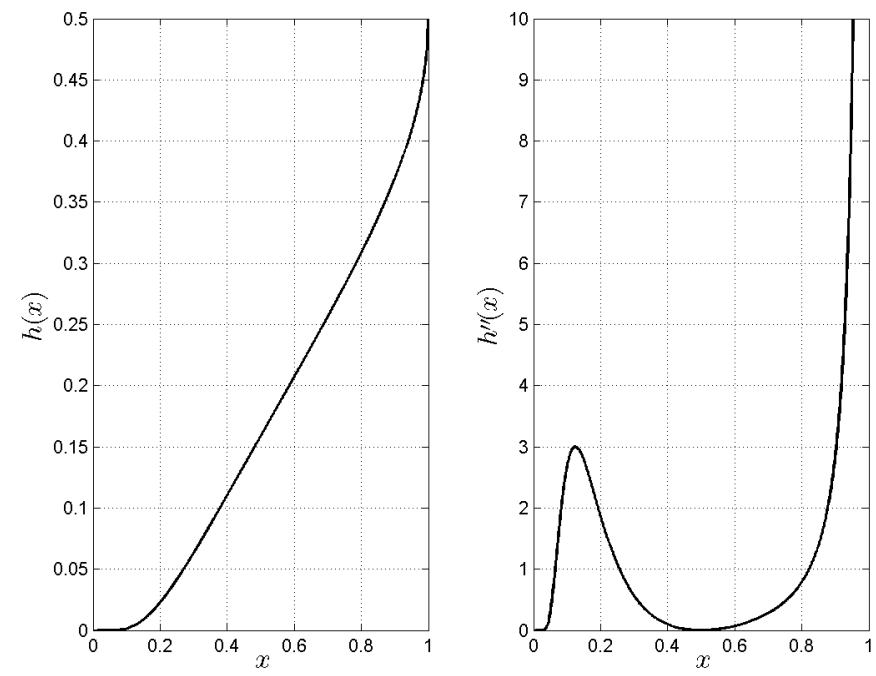

Figura 4.1: Função $h(x)$ e sua segunda derivada para modulação QPSK.

não-positivos. Primeiramente, note que $a(x) \leq 0$ equivale a:

$$
4 x^{2}-(\beta+3) x+\beta \leq 0
$$

Essa inequação quadrática tem como solução:

$$
\frac{\beta+3-\sqrt{\beta^{2}-10 \beta+9}}{8} \leq x \leq \frac{\beta+3+\sqrt{\beta^{2}-10 \beta+9}}{8}
$$

Mas a função $h(x)$ é calculada em $x_{k}=\frac{1}{1+\gamma\left|H_{k}\right|^{2}}$, desse modo, a condição para que a $k$-ésima componente subportadora esteja na região de concavidade é dada por:

$$
\underbrace{\frac{\beta+3-\sqrt{\beta^{2}-10 \beta+9}}{8}}_{L_{i}(\beta)} \leq \frac{1}{1+\gamma\left|H_{k}\right|^{2}} \leq \underbrace{\frac{\beta+3+\sqrt{\beta^{2}-10 \beta+9}}{8}}_{L_{s}(\beta)}
$$

ou ainda:

$$
\frac{1}{L_{s}(\beta)}-1 \leq \gamma\left|H_{k}\right|^{2} \leq \frac{1}{L_{i}(\beta)}-1
$$

A Fig. 4.2 mostra os intervalos de concavidade e convexidade em função da cardinalidade da modulação. Como já analisado, no caso QPSK, a função $h(x)$ é convexa em todo intervalo de definição. A medida que a ordem da modulação aumenta, a região de concavidade cresce.

Apesar da Fig. 4.2 indicar os valores de $\gamma\left|H_{k}\right|^{2}$ para os quais a função $h(x)$ é convexa, ela não fornece nenhum indicativo a respeito de quanto diferem as probabilidades de erro. 


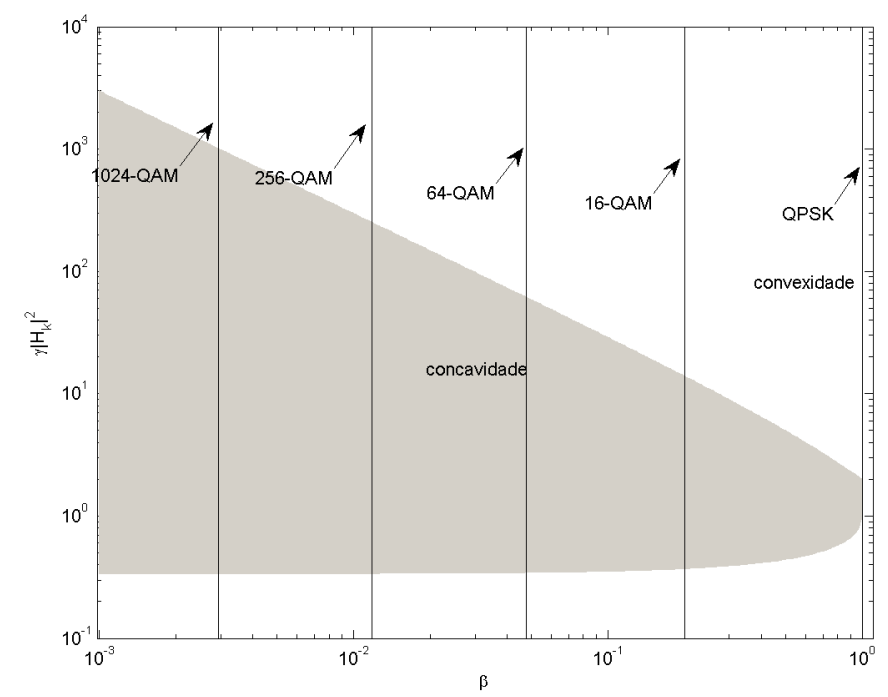

Figura 4.2: Regiões de concavidade e convexidade de $h(x)$ em função da modulação adotada.

Para tanto, é necessário analisar a magnitude da segunda derivada da função. Não é difícil demonstrar que a magnitude da segunda derivada está relacionada com a diferença existente entre a função calculada no valor médio de $\left.x_{k}\right|_{k=0} ^{N-1}$, i.e, $h\left(\frac{1}{N} \sum_{k=0}^{N-1} x_{k}\right)$ e o valor médio da função calculada em $x_{k}$ i.e, $\frac{1}{N} \sum_{k=0}^{N-1} h\left(x_{k}\right)$.

A Fig. 4.3 mostra que, à medida que $x_{k}$ tende a $x_{k}=1$, o valor da segunda derivada aumenta independentemente da cardinalidade de modulação empregada. Observe, entretanto, que para $\gamma$ fixado, a única condição para $x_{k} \rightarrow 1$ é $H_{k} \rightarrow 0$. Essa observação é coerente com o fato já conhecido de que em condições de nulo espectral, o sistema OFDM não-codificado não apresenta bons resultados, já que a BER associada à portadora desvanecida tenderá a $1 / 2$, comprometendo todo o sistema.

Com exceção do caso de nulo espectral, note que se $\gamma \rightarrow \infty, x_{k} \rightarrow 0 \forall k$. Nessas condições, todas as subportadoras estarão na região de convexidade, validando a eq. (4.22). Em outras palavras, mesmo que BER $\mathrm{BFDM}_{O \mathrm{BER}_{L E}}$ em regime de baixa SNR, há um limiar de $\gamma$, tal que $\gamma>\gamma_{\text {lim }} \rightarrow \mathrm{BER}_{O F D M}>\mathrm{BER}_{L E}$.

Para que todas as subportadoras estejam na região de convexidade da função, é suficiente que:

$$
x_{k} \leq L_{i}(\beta), \quad k=0, \ldots, N-1
$$

ou ainda:

$$
\frac{1}{1+\gamma\left|H_{k}\right|^{2}} \leq L_{i} \Leftrightarrow \gamma \geq \frac{1}{\left|H_{k}\right|^{2}}\left(\frac{1}{L_{i}}-1\right)
$$




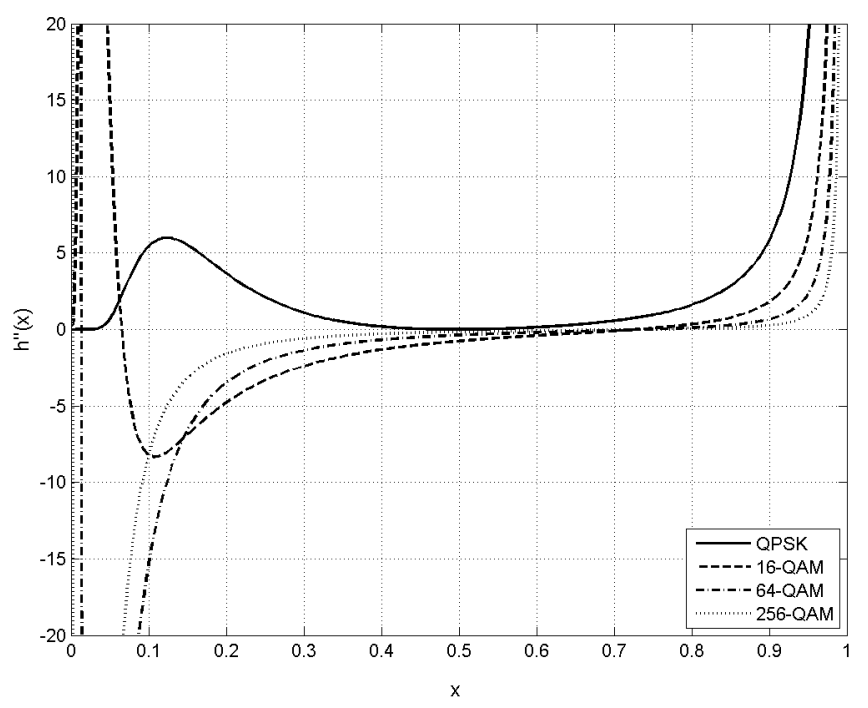

Figura 4.3: Segunda derivada da função $h(x)$.

Concluindo, o limiar de SNR é:

$$
\gamma_{l i m}=\max _{k}\left(\frac{1}{\left|H_{k}\right|^{2}}\left(\frac{1}{L_{i}}-1\right)\right)
$$

É ainda importante ressaltar que apesar de $\gamma \leq \gamma_{\text {lim }}$ garantir que $\mathrm{BER}_{O F D M}>\mathrm{BER}_{L E}$, a recíproca não é verdadeira. É possível que, mesmo para SNRs abaixo de $\gamma_{l i m}$, o sistema LE-SCCP apresente um desempenho melhor do que o desempenho apresentado pelo OFDM. Uma vez que nas situações em que há algumas subportadoras na região de concavidade e outras na região de convexidade, não é possível chegar a qualquer relação fechada entre as BERs dos referidos sistemas.

A fim de validar o tratamento teórico desenvolvido, será analisado o desempenho dos sistemas quando submetidos a um canal seletivo em frequência com função transferência dada por:

$$
H(z)=0,0854+0,8544 z^{-1}+0,5126 z^{-2}
$$

Note que, no caso QPSK, a Fig. 4.4 mostra que o desempenho do SCCP é superior ao do OFDM independentemente da SNR. Para modulações de cardinalidade mais elevada, o sistema OFDM apresenta uma BER menor que o sistema LE-SCCP, em certas condições de SNR. Note ainda que, à medida que a ordem da modulação aumenta, o limiar de SNR, que determina as regiões em que o OFDM apresenta desempenho superior em relação ao LE-SCCP, aumenta. Todos essas fatos corroboram a análise teórica desenvolvida. 


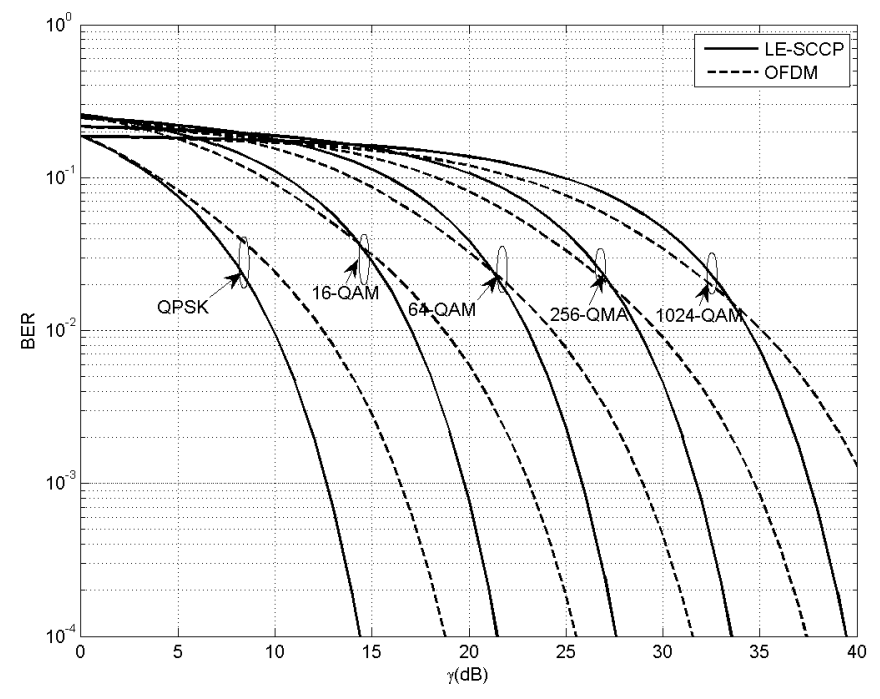

Figura 4.4: Comparação de desempenho, sem codificação de canal, entre os sistemas OFDM e LE-SCCP para o canal com função de transferência dada pela eq.(4.31).

\subsubsection{OFDM vs DFE-SCCP}

Como mostrado no capítulo 3, o LE-SCCP sempre apresenta um desempenho inferior ao do DFE-SCCP perfeito. Já foi demonstrado que no caso QPSK, o LE-SCCP sempre apresenta desempenho superior ao do OFDM. Logo, concluímos que o DFE-SCCP sempre leva a uma BER menor do que a do OFDM para a modulação QPSK.

Em relação ao 16-QAM, mostrou-se através da análise de convexidade da função $h(x)$ que há situações em que o OFDM ganha em desempenho quando comparado ao LE-SCCP. A pergunta que surge é se o DFE consegue suprir essa degradação exibida pelo filtro linear quando modulações de maior cardinalidade são utilizadas.

Na Fig. 4.5, com o objetivo de fornecer subsídio à questão, comparamos o desempenho do DFE-SCCP com o LE-SCCP e o OFDM quando a transmissão se dá no canal com função de transferência dada pela eq. (4.31), utilizando-se modulação 16-QAM. Pode-se observar que o DFE possui desempenho superior a todas as demais técnicas para a região de SNR simulada.

Pode-se questionar se esse mesmo comportamento seria observado para outras configurações de canal e outras modulações. A fim de responder a esta pergunta, pode-se aplicar a mesma análise de convexidade utilizada na comparação entre o OFDM e o LE-SCCP à comparação entre o OFDM e o DFE-SCCP. Para tanto, serão utilizados os mapeamentos 


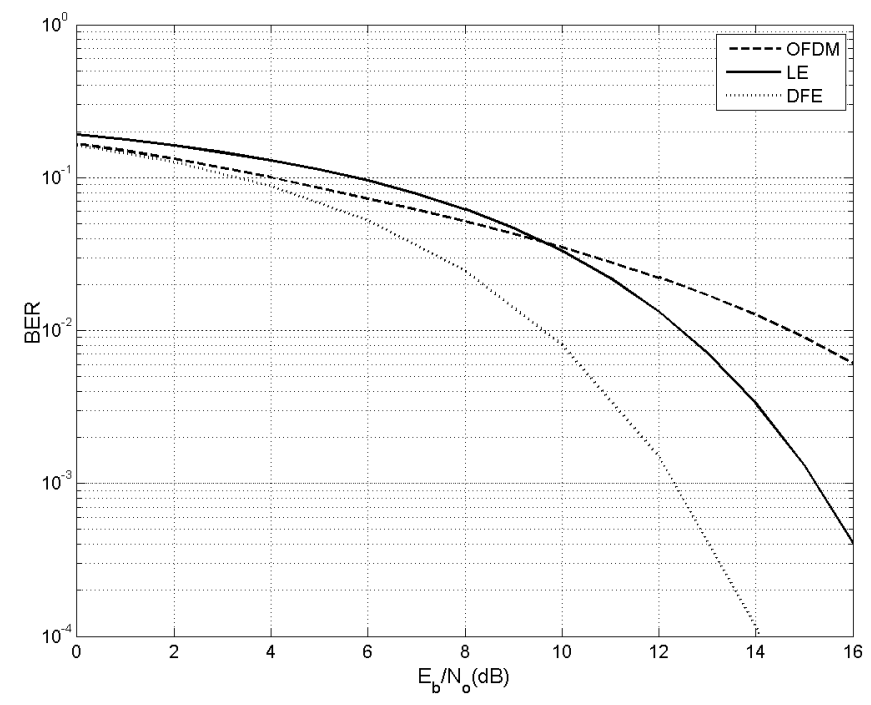

Figura 4.5: Transmissão 16-QAM sem codificação de canal, incluindo a equalização DFE. Canal com função transferência dada pela eq. (4.31).

$t(x)$ e $\phi(x)$, tal que:

$$
\mathrm{SNR}_{D F E}=t\left(\frac{1}{N} \sum_{k=0}^{N-1} \phi\left(\gamma\left|H_{k}\right|^{2}\right)\right) \quad \operatorname{SNR}_{O F D M}=\frac{1}{N} \sum_{k=0}^{N-1} t\left(\phi\left(\gamma\left|H_{k}\right|^{2}\right)\right)
$$

sendo $t(x)$ uma função convexa e $\phi(x)$ uma função arbitrária da SNR em cada subportadora.

Sabendo que a BER do DFE-SCCP é dada por:

$$
\mathrm{BER}_{D F E}=\alpha Q\left(\sqrt{\beta \mathrm{SNR}_{D F E}}\right)
$$

e que a $\mathrm{SNR}_{D F E}$ pode ser escrita como indicado na eq. (3.59), tem-se:

$$
\mathrm{BER}_{D F E}=\alpha Q\left(\sqrt{\beta\left(\text { geomean }\left(1+\gamma|\mathbf{H}|^{2}\right)-1\right)}\right)
$$

e definindo $\psi(x)=\alpha Q(\sqrt{\beta x})$, a expressão acima pode ser expressa como:

$$
\operatorname{BER}_{D F E}=\psi\left(\text { geomean }\left(1+\gamma|\mathbf{H}|^{2}\right)-1\right)
$$


Lembrando que a média geométrica pode ser expressa como:

$$
\operatorname{geomean}(\mathbf{x})=\exp \left(\sum_{k=0}^{N-1} \log \left(x_{k}\right)\right)
$$

pode-se definir $\phi(x)=\log (x+1)$ com inversa $\phi^{-1}(x)=\exp (x)-1$, dessa forma:

$$
\mathrm{BER}_{D F E}=\psi\left\{\phi^{-1}\left(\sum_{k=0}^{N-1} \phi\left(\gamma\left|H_{k}\right|^{2}\right)\right)\right\}
$$

Voltando as atenções ao OFDM, a eq. (4.8) mostra que a BER para esse sistema pode ser escrita como:

$$
\mathrm{BER}_{O F D M}=\frac{1}{N} \sum_{k=0}^{N-1} \psi\left(\gamma\left|H_{k}\right|^{2}\right)
$$

Com o objetivo de comparar as eqs. (4.37) e (4.38), define-se a seguinte função:

$$
t(y)=\psi\left(\phi^{-1}(y)\right)
$$

Substituindo a eq. (4.39) na eq. (4.37), tem-se:

$$
\mathrm{BER}_{D F E}=t\left(\frac{1}{N} \sum_{k=0}^{N-1} \phi\left(\gamma\left|H_{k}\right|^{2}\right)\right)
$$

Ainda, a partir da eq. (4.39), tem-se que $\psi\left(\gamma\left|H_{k}\right|^{2}\right)=t\left(\phi\left(\gamma\left|H_{k}\right|^{2}\right)\right)$. Finalmente, a eq. (4.38) pode ser reescrita como:

$$
\mathrm{BER}_{O F D M}=\frac{1}{N} \sum_{k=0}^{N-1} t\left(\phi\left(\gamma\left|H_{k}\right|^{2}\right)\right)
$$

Definindo:

$$
y_{k}=\phi\left(\gamma\left|H_{k}\right|^{2}\right)=\log \left(1+\gamma\left|H_{k}\right|^{2}\right)
$$


as eqs. (4.40) e (4.41) podem ser expressas como:

$$
\begin{aligned}
& \mathrm{BER}_{D F E}=t\left(\frac{1}{N} \sum_{k=0}^{N-1} y_{k}\right) \\
& \mathrm{BER}_{O F D M}=\frac{1}{N} \sum_{k=0}^{N-1} t\left(y_{k}\right)
\end{aligned}
$$

Se a função $t(y)$ for convexa, é possível aplicar a desigualdade de Jensen e provar que $\mathrm{BER}_{D F E} \leq \mathrm{BER}_{O F D M}$.

Agora que ambas as BER estão escritas em função de $t(y)$, precisamos analisar a função $t(y)$ em detalhes:

$$
t(y)=\psi\left(\phi^{-1}(y)\right)=\alpha Q(\sqrt{\beta(\exp (y)-1)})
$$

Assim, a primeira derivada da função $t(y)$ é dada por:

$$
\frac{d}{d y} t(y)=-\frac{\alpha \beta}{\sqrt{2 \pi}} \frac{1}{2 \sqrt{\beta\left(e^{y}-1\right)}} e^{\left(y-\frac{1}{2}\left(\beta\left(e^{y}-1\right)\right)\right)}
$$

e sua segunda derivada é dada por:

$$
\frac{d^{2}}{d y^{2}} t(y)=\underbrace{\left(1-\frac{\beta e^{y}}{2}\left(1+\frac{1}{\beta\left(e^{y}-1\right)}\right)\right)}_{b(y)} \frac{d}{d y} t(y)
$$

Como $\frac{d}{d y} t(y)$ é uma função estritamente negativa, uma condição para que $\frac{d^{2}}{d y^{2}} t(y)$ seja negativa, i.e., a função $t(y)$ apresente regiões de concavidade, é que a função $b(y)$ seja positiva para algum $y$. Como descrito no apêndice $B$, tal fato é observado se $\beta<3-2 \sqrt{2}$ ou $\beta>3+2 \sqrt{2}$.

Lembrando que para uma constelação de cardinalidade $M, \beta=\frac{3}{M-1}$, conclui-se que $\beta \leq 1$, para qualquer modulação adotada. Dessa forma, a condição $\beta>3+2 \sqrt{2}$ não tem significado físico.

Observemos para quais valores de $M$, tem-se $\beta<3-2 \sqrt{2}$.

$$
\beta=\frac{3}{M-1}<3-2 \sqrt{2} \Rightarrow M>\frac{3}{3-2 \sqrt{2}}+1 \approx 18,48
$$

Concluímos que para modulações QPSK e 16-QAM, o DFE perfeito apresentará um desempenho superior ao do OFDM para qualquer condição de canal. Já para modulações 
com cardinalidade mais elevadas, a função $t(y)$ apresentará uma região de concavidade dada por:

$$
\log \left(\frac{(\beta+1)-\sqrt{(1+\beta)^{2}-8 \beta}}{2 \beta}\right) \leq y \leq \log \left(\frac{(\beta+1)+\sqrt{(1+\beta)^{2}-8 \beta}}{2 \beta}\right)
$$

Mas pelas eqs. (4.40) e (4.41), as expressões de BER para ambos os sistemas dependem do valor da função $t(y)$ calculada em $y_{k}=\phi\left(\gamma\left|H_{k}\right|^{2}\right)$. Portanto, a condição para que a $k$-ésima componente em frequência opere na região de concavidade é dada por:

$$
\log \left(\frac{(\beta+1)-\sqrt{(1+\beta)^{2}-8 \beta}}{2 \beta}\right) \leq \log \left(1+\gamma\left|H_{k}\right|^{2}\right) \leq \log \left(\frac{(\beta+1)+\sqrt{(1+\beta)^{2}-8 \beta}}{2 \beta}\right)
$$

ou ainda:

$$
\frac{(\beta+1)-\sqrt{(1+\beta)^{2}-8 \beta}}{2 \beta}-1 \leq \gamma\left|H_{k}\right|^{2} \leq \frac{(\beta+1)+\sqrt{(1+\beta)^{2}-8 \beta}}{2 \beta}-1
$$

e definindo:

$$
\begin{aligned}
& L_{i}(\beta)=\frac{(\beta+1)-\sqrt{(1+\beta)^{2}-8 \beta}}{2 \beta}-1 \\
& L_{s}(\beta)=\frac{(\beta+1)+\sqrt{(1+\beta)^{2}-8 \beta}}{2 \beta}-1
\end{aligned}
$$

tem-se:

$$
L_{i}(\beta) \leq \gamma\left|H_{k}\right|^{2} \leq L_{s}(\beta)
$$

Alguns fatores merecem destaque na expressão acima. Primeiramente, a Fig. 4.6 mostra que, à medida que o nível de modulação aumenta, i.e. $\beta \rightarrow 0$, a região de concavidade aumenta. Isso significa que, com o aumento da cardinalidade da modulação, as situações em que o OFDM apresenta desempenho superior ao DFE passam a ser mais frequentes.

Além disso, note que para forçar que todas as subportadoras operem fora da região de concavidade, basta fazer com que a relação sinal ruído em cada subportadora seja superior a $L_{s}(\beta)$. Logo, mesmo que, a princípio, o DFE possa apresentar um desempenho inferior ao do OFDM para baixas SNRs, a BER do OFDM supera a do DFE para SNRs mais elevadas.

Até então, foram analisadas apenas quais situações de canal levam os sistemas a operar nas regiões de convexidade ou nas regiões de concavidade da função $t(y)$. Concluiu-se que $\mathrm{BER}_{D F E} \leq \mathrm{BER}_{O F D M}$ nas regiões de convexidade e $\mathrm{BER}_{D F E} \geq \mathrm{BER}_{O F D M}$ nas regiões de 


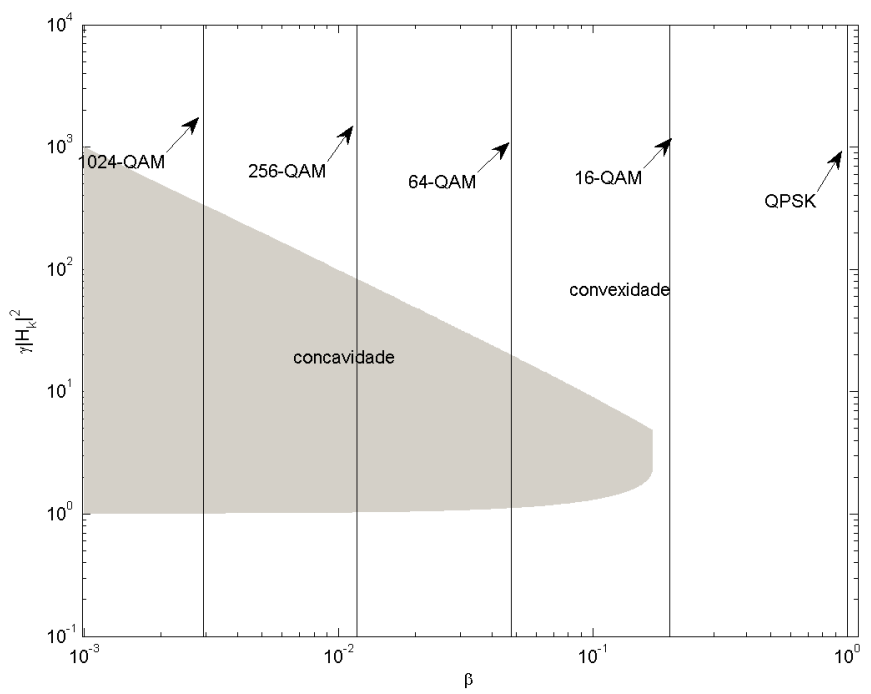

Figura 4.6: Região de concavidade da função $t(y)$ para diferentes valores de $\beta$.

concavidade. Entretanto, ainda é necessário avaliar de quanto diferem as probabilidades de erro dos dois sistemas.

A Fig. 4.7 mostra a magnitude da segunda derivada da função $t(y)$. Veja que, conforme demonstrado anteriormente, nos casos QPSK e 16-QAM, a função é convexa em todo intervalo de domínio. Com o aumento da cardinalidade da modulação, a função passa a apresentar regiões de concavidade. Entretanto, nessas regiões de concavidade, a magnitude de $\frac{d^{2}}{d y^{2}} t(y)$ é muito pequena. Logo, nos casos em que o OFDM se mostra superior ao DFE, a diferença entre os dois sistemas é muito sutil. Já nas situações em que o DFE é superior ao OFDM, a magnitude de $\frac{d^{2}}{d y^{2}} t(y)$ é muito maior do que nas regiões de concavidade. Nesse caso, a diferença entre os dois sistemas é considerável. Veja que essa situação ocorre se $y_{k}=\phi\left(\gamma\left|H_{k}\right|^{2}\right) \rightarrow 0$ ou $\log \left(1+\gamma\left|H_{k}\right|^{2}\right) \rightarrow 0$, i.e., se o canal apresenta nulos espectrais.

A fim de verificar o tratamento teórico desenvolvido, mais uma vez será considerado o canal com resposta impulsiva dada pela eq.(4.31). Os resultados estão mostrados na Fig. 4.8. Assim como previsto no tratamento teórico, o sistema DFE-SCCP apresenta desempenho superior ao do OFDM para as modulação QPSK e 16-QAM. A partir da modulação 64QAM, para valores baixos de SNR, o desempenho do OFDM passa a ser um pouco superior ao desempenho do DFE-SCCP. 


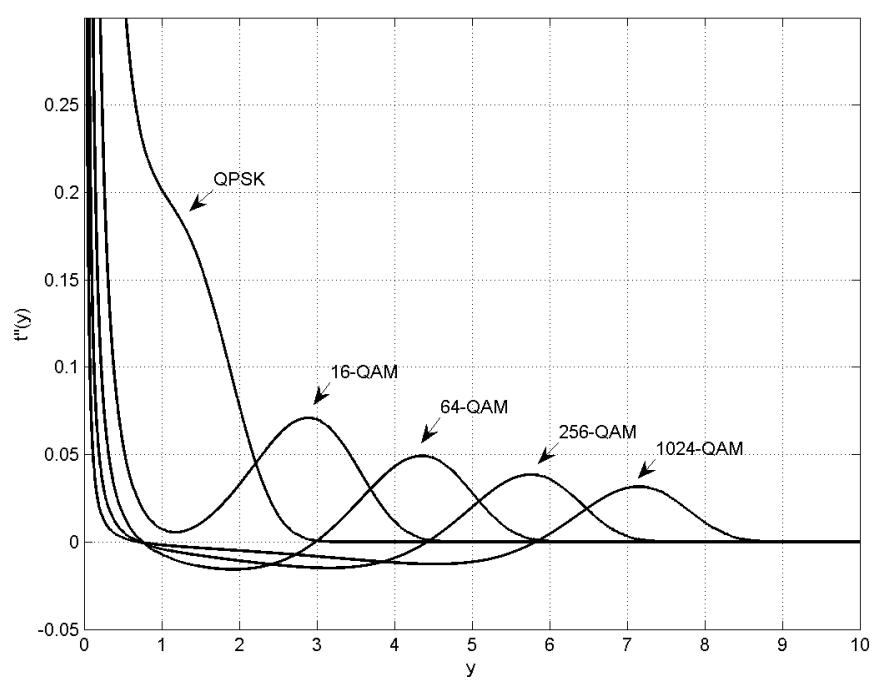

Figura 4.7: Segunda derivada da função $t(y)$.

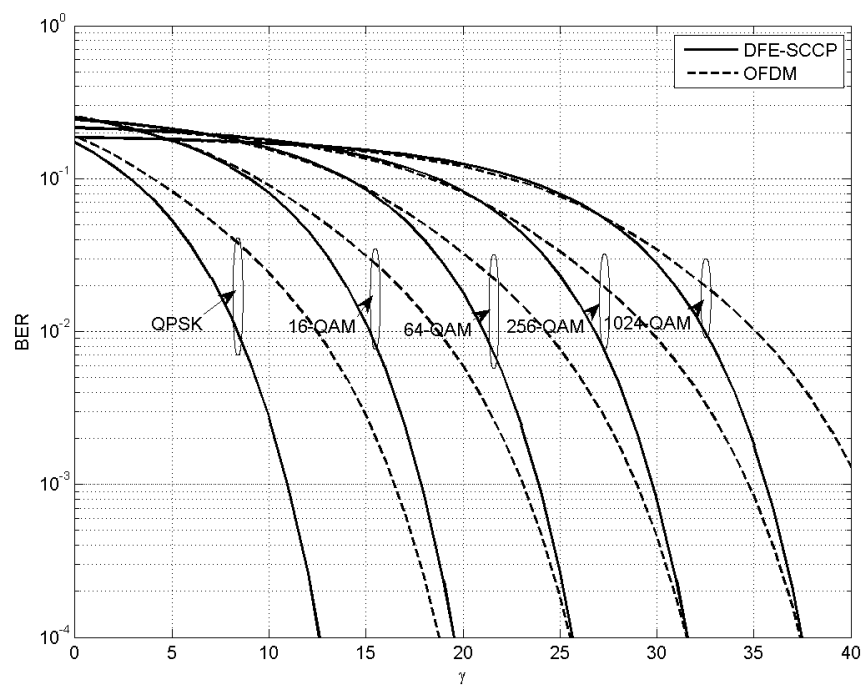

Figura 4.8: Comparação de desempenho, sem codificação de canal, entre os sistemas OFDM e DFE-SCCP para o canal com função de transferência dada pela eq.(4.31). 


\section{Sumário}

Neste capítulo os sistemas OFDM e SCCP foram comparados no contexto sem codificação de canal.

Para modulação QPSK, foi demonstrado que o sistema OFDM perde em desempenho para o sistema LE-SCCP em qualquer condição de canal. Esse resultado já havia sido apresentado em [23].

Posteriormente, a comparação foi estendida para modulação 16-QAM. Nesse contexto, mostrou-se que há certas condições de canal para as quais o LE-SCCP apresenta um desempenho pior que o OFDM. Além disso, foi provado que se o sinal for equalizado com um DFE, o sistema SCCP passa a apresentar um desempenho melhor que o OFDM. Para modulações de cardinalidade ainda mais elevada, foi provado que existem situações nas quais o sistema SCCP apresenta pior desempenho que o sistema OFDM, mesmo se equalizado com um DFE perfeito.

Finalmente, a principal conclusão do capítulo reside no fato de que o aumento na ordem da modulação tende a degradar mais o sistema SCCP que o sistema OFDM. No próximo capítulo, será analisado como os sistemas se comportam a partir do momento em que codificação de canal é utilizada na transmissão. 


\section{Capítulo 5}

\section{Sensibilidade em relação à taxa de codificação}

No capítulo anterior, foram comparados os sistemas OFDM e SCCP do ponto de vista da cardinalidade da constelação adotada no mapeamento de bits. Já neste capítulo, será analisada a questão da taxa de codificação de canal, que é de fundamental importância e merece especial atenção.

A análise de sistemas codificados não é uma tarefa trivial. Neste capítulo ela será realizada de um ponto de vista teórico, a partir de algumas hipóteses que não são observadas em situações reais. Já no próximo capítulo, a partir de simulação Monte Carlo, abordaremos o problema nos fundamentando em restrições de ordem mais práticas, e confrontaremos os resultados obtidos nesse contexto com o desenvolvimento teórico que será apresentado neste capítulo.

A análise teórica será pautada através de dois conceitos distintos. Primeiramente, os conceitos de capacidade de canal de Shannon serão aplicados e em seguida ambos os sistemas serão comparados em termos de probabilidade de outage por cutoff rate.

\subsection{Capacidade de Shannon}

Para o cálculo da capacidade do sistema DFE-SCCP, mais uma vez será considerado que o equalizador DFE é perfeito, i.e. livre de propagação de erros.

Como indicado no capítulo anterior e por conveniência aqui reproduzido, a SNR na saída 
do equalizador DFE quando a transmissão se dá com prefixo cíclico é dada por:

$$
\mathrm{SNR}=\frac{1}{J_{\min }}-1=\exp \left\{\frac{1}{N} \sum_{k=0}^{N-1} \log \left(1+\gamma\left|H_{k}\right|^{2}\right)\right\}-1
$$

A partir da SNR, pode-se calcular a capacidade do sistema:

$$
C=\log _{2}(1+\mathrm{SNR})=\frac{1}{N} \sum_{k=0}^{N-1} \log _{2}\left(1+\gamma\left|H_{k}\right|^{2}\right)
$$

Conclusão:

$$
C_{D F E}=\frac{1}{N} \sum_{k=0}^{N-1} \log _{2}\left(1+\gamma\left|H_{k}\right|^{2}\right)
$$

No caso OFDM, como a SNR em cada subportadora é dada por $\mathrm{SNR}_{k}=\gamma\left|H_{k}\right|^{2}$, considerando ergodicidade [53] tem-se que a capacidade é expressa por:

$$
C_{O F D M}=\frac{1}{N} \sum_{k=0}^{N-1} \log _{2}\left(1+\mathrm{SNR}_{k}\right)=\frac{1}{N} \sum_{k=0}^{N-1} \log _{2}\left(1+\gamma\left|H_{k}\right|^{2}\right)
$$

Lembrando que a banda ocupada por ambos os sistemas é a mesma, a capacidade do sistema OFDM é igual ao do SCCP quando equalizado com o DFE.

Como considera-se o DFE perfeito, a SNR na saída do equalizador é sempre maior do que a SNR na saída do equalizador implementado com filtro linear. Dessa forma, a seguinte desigualdade é válida:

$$
C_{L E} \leq C_{D F E}=C_{O F D M}
$$

O fato de ambos os sistemas apresentarem a mesma capacidade não implica que eles tenham desempenhos equivalentes quando codificados de maneira prática, uma vez que o impacto da codificação é sentido de maneira distinta nos casos OFDM e SCCP.

A aplicação de capacidade de canal para o estudo do desempenho de sistemas codificados, apesar de ser uma ferramenta extremamente importante, não leva em consideração alguns fatores fundamentais. Uma dessas lacunas deixada pela análise de capacidade de canal diz respeito à cardinalidade da modulação empregada no sistema. Tal lacuna pode ser superada pela cutoff rate [54]. 


\subsection{Análise através da cutoff rate}

A cutoff rate é um parâmetro que avalia o desempenho do sistema codificado, levando em conta a modulação usada no mapeamento de bits. Comparando com a capacidade de canal, é ainda possível mostrar que a cutoff rate pode ser vista como um limitante inferior da capacidade de Shannon [55].

Esse parâmetro é analisado em um dos poucos artigos com resultados teóricos na área de comparação entre o OFDM e SCCP, a publicação de Aue et al. [25]. Nesse artigo, a cutoff rate é empregada para a comparação dos sistemas OFDM e LE-SCCP em canais com dois coeficientes Rayleigh e perfil de potência uniforme com modulação QPSK.

Nesta seção, serão deduzidas as expressões de cutoff rate para os sistemas estudados, e o estudo será estendido ao caso em que a transmissão se dá empregando constelações de cardinalidades mais elevadas. Além disso, serão considerados sistemas DFE-SCCP.

\subsubsection{Expressões para cutoff rate}

Em [54], é deduzida uma expressão para a cutoff rate a partir da aplicação da union bound. Para o caso de transmissão com uma constelação $M$-ária, de símbolos $x_{l}$, esse parâmetro é dado por:

$$
R_{0}=-\log _{2}\left(\frac{1}{M^{2}} \sum_{l=0}^{M-1} \sum_{m=0}^{M-1} \mathbb{P}\left\{x_{l} \mapsto x_{m}\right\}\right)
$$

em que $\mathbb{P}\left\{x_{l} \mapsto x_{m}\right\}$ representa a pairwise error probability (PEP), i.e. a probalidade de decodificar o símbolo como $x_{m}$ dado que $x_{l}$ foi transmitido.

Em canais AWGN, tem-se que:

$$
\mathbb{P}\left\{x_{l} \mapsto x_{m}\right\}=Q\left(\sqrt{\frac{\left\|x_{l}-x_{m}\right\|^{2}}{2 N_{o}}}\right)
$$

Mas, $Q(x)<\exp ^{\frac{x^{2}}{2}}$, (cota de Chernoff). Utilizando essa aproximação, a cutoff rate pode ser expressa como:

$$
R_{0, A W G N}=-\log _{2}\left(\frac{1}{M^{2}} \sum_{l=0}^{M-1} \sum_{m=0}^{M-1} \exp \left\{-\frac{\left\|x_{l}-x_{m}\right\|^{2}}{4 N_{o}}\right\}\right)
$$

A constante $\left\|x_{l}-x_{m}\right\|$ representa a distância euclidiana entre os símbolos $x_{l}$ e $x_{m}$. Pode- 
se escrever esse parâmetro como um múltiplo da distância mínima entre os símbolos da constelação, $\left\|x_{l}-x_{m}\right\|=\sqrt{A_{l, m}} d_{m i n}$. No caso de modulação M-QAM, a distância mínima é dada por [52]:

$$
d_{\text {min }}=\sqrt{2 \frac{3}{M-1} \log _{2}(M) E_{b}}
$$

Definindo a taxa de codificação como $R$ e reconhecendo $\beta=\frac{3}{M-1}$, tem-se:

$$
\frac{\left\|x_{l}-x_{m}\right\|^{2}}{N_{o}}=2 \beta A_{l, m} \log _{2}(M) \frac{E_{b}}{N_{o}}=\frac{2 \beta A_{l, m}}{R} \frac{\sigma_{x}^{2}}{\sigma_{v}^{2}}
$$

Definindo $\gamma=\frac{\sigma_{x}^{2}}{\sigma_{v}^{2}}$, a cutoff rate passa a ser dada por:

$$
R_{0}=-\log _{2}\left(\frac{1}{M^{2}} \sum_{l=0}^{M-1} \sum_{m=0}^{M-1} \exp \left\{-\frac{\beta A_{l, m}}{2 R} \gamma\right\}\right)
$$

Em canais com desvanecimento plano, i.e., canais com resposta impulsiva da forma $h=$ $a, a \sim \operatorname{eN}\left(0, \sigma_{a}^{2}\right)$, a cutoff rate passa a ser calculada como:

$$
R_{0}=-\log _{2}\left(\frac{1}{M^{2}} \sum_{l=0}^{M-1} \sum_{m=0}^{M-1} \mathrm{E}\left\{\exp \left\{-a^{2} \frac{\beta A_{l, m}}{2 R} \gamma\right\}\right\}\right)
$$

A partir da eq.(5.12), pode-se calcular a cutoff rate para o sistema OFDM em canais seletivos em frequência, lembrando que a transmissão OFDM pode ser vista como um caso de transmissão em canais gaussianos em paralelo. Como o ganho de cada subcanal é dado pela magnitude da resposta em frequência associada à subportadora, a cutoff rate para o sistema OFDM é dada por:

$$
R_{0, \text { OFDM }}=-\log _{2}\left(\frac{1}{M^{2}} \sum_{l=0}^{M-1} \sum_{m=0}^{M-1} \mathrm{E}\left\{\exp \left\{-\frac{\beta A_{l, m}}{2 R}\left|H_{k}\right|^{2} \gamma\right\}\right\}\right)
$$

Por ergodicidade, a cutoff rate do sistema OFDM pode ser escrita como:

$$
R_{0, \text { OFDM }}=-\log _{2}\left(\frac{1}{M^{2}} \sum_{l=0}^{M-1} \sum_{m=0}^{M-1} \frac{1}{N} \sum_{k=0}^{N-1}\left[\exp \left\{-\gamma \frac{\beta A_{l, m}}{2 R}\left|H_{k}\right|^{2}\right\}\right]\right)
$$


No caso SCCP, dada a SNR na saída do equalizador, a cutoff rate é dada por:

$$
R_{0, S C C P}=-\log _{2}\left(\frac{1}{M^{2}} \sum_{l=0}^{M-1} \sum_{m=0}^{M-1} \exp \left\{-\operatorname{SNR} \frac{\beta A_{l, m}}{2 R}\right\}\right)
$$

A SNR depende do tipo de equalização utilizada. Por conveniência, as expressões para as SNRs nas saídas do equalizador linear e do equalizador DFE são reproduzidas a seguir:

$$
\begin{gathered}
\mathrm{SNR}_{L E}=\frac{\sum_{k=0}^{N-1} \frac{\gamma\left|H_{k}^{2}\right|}{\gamma\left|H_{k}\right|^{2}+1}}{\sum_{k=0}^{N-1} \frac{1}{\gamma\left|H_{k}\right|^{2}+1}} \\
\mathrm{SNR}_{D F E}=\exp \left\{\frac{1}{N} \sum_{k=0}^{N-1} \log \left(1+\gamma\left|H_{k}\right|^{2}\right)\right\}-1
\end{gathered}
$$

Assim como feito no cap.4, as eqs. (5.16) e (5.17) são reescritas a partir das funções auxiliares $\phi(x)=\log (x+1)$ e $\theta(x)=\frac{1}{x+1}$ :

$$
\begin{gathered}
\operatorname{SNR}_{L E}=\theta^{-1}\left(\frac{1}{N} \sum_{k=0}^{N-1} \theta\left(\gamma\left|H_{k}\right|^{2}\right)\right) \\
\operatorname{SNR}_{D F E}=\phi^{-1}\left(\frac{1}{N} \sum_{k=0}^{N-1} \phi\left(\gamma\left|H_{k}\right|^{2}\right)\right)
\end{gathered}
$$

Então, as expressões para as cutoff rates dos sistemas SCCP podem ser reescritas como:

$$
\begin{aligned}
R_{0, L E} & =-\log _{2}\left(\frac{1}{M^{2}} \sum_{l=0}^{M-1} \sum_{m=0}^{M-1} \exp \left\{-\frac{\beta A_{l, m}}{2 R} \theta^{-1}\left(\frac{1}{N} \sum_{k=0}^{N-1} \theta\left(\gamma\left|H_{k}\right|^{2}\right)\right)\right\}\right. \\
R_{0, D F E} & =-\log _{2}\left(\frac{1}{M^{2}} \sum_{l=0}^{M-1} \sum_{m=0}^{M-1} \exp \left\{-\frac{\beta A_{l, m}}{2 R} \phi^{-1}\left(\frac{1}{N} \sum_{k=0}^{N-1} \phi\left(\gamma\left|H_{k}\right|^{2}\right)\right)\right\}\right)
\end{aligned}
$$

Analisando as eqs. (5.14), (5.20) e (5.21), conclui-se que para se comparar as cutoff rates 
dos sistemas LE-SCCP, DFE-SCCP e OFDM é suficiente comparar as seguintes funções:

$$
\begin{array}{r}
\zeta_{O F D M}(l, m)=\frac{1}{N} \sum_{k=0}^{N-1}\left[\exp \left\{-\gamma \frac{\beta A_{l, m}}{2 R}\left|H_{k}\right|^{2}\right\}\right] \\
\zeta_{L E}(l, m)=\exp \left\{-\frac{\beta A_{l, m}}{2 R} \theta^{-1}\left(\frac{1}{N} \sum_{k=0}^{N-1} \theta\left(\gamma\left|H_{k}\right|^{2}\right)\right)\right\} \\
\zeta_{D F E}(l, m)=\exp \left\{-\frac{\beta A_{l, m}}{2 R} \phi^{-1}\left(\frac{1}{N} \sum_{k=0}^{N-1} \phi\left(\gamma\left|H_{k}\right|^{2}\right)\right)\right\}
\end{array}
$$

Reescrevendo as expressões de cutoff rate em função dessas grandezas, tem-se:

$$
\begin{array}{r}
R_{0, O F D M}=-\log _{2}\left(\frac{1}{M^{2}} \sum_{l=0}^{M-1} \sum_{m=0}^{M-1} \zeta_{\text {OFDM }}(l, m)\right) \\
R_{0, L E}=-\log _{2}\left(\frac{1}{M^{2}} \sum_{l=0}^{M-1} \sum_{m=0}^{M-1} \zeta_{L E}(l, m)\right) \\
R_{0, D F E}=-\log _{2}\left(\frac{1}{M^{2}} \sum_{l=0}^{M-1} \sum_{m=0}^{M-1} \zeta_{D F E}(l, m)\right)
\end{array}
$$

Logo, comparar as cutoff rates equivale a comparar as grandezas $\zeta$. Pela monotonicidade decrescente da função $-\log (x)$, se $\zeta_{a}<\zeta_{b}, R_{0, a}>R_{0, b}$.

Procedendo com a comparação entre as funções $\zeta$, define-se mais uma função auxiliar, $\tau(x)=\exp \left(-\frac{\beta A_{l, m}}{2 R} x\right)$, de forma que as expressões para as grandezas $\zeta$ possam ser reescritas como:

$$
\begin{array}{r}
\zeta_{O F D M}=\frac{1}{N} \sum_{k=0}^{N-1} \tau\left(\gamma\left|H_{k}\right|^{2}\right) \\
\zeta_{L E}=\tau\left(\theta^{-1}\left(\frac{1}{N} \sum_{k=0}^{N-1} \theta\left(\gamma\left|H_{k}\right|^{2}\right)\right)\right) \\
\zeta_{D F E}=\tau\left(\phi^{-1}\left(\frac{1}{N} \sum_{k=0}^{N-1} \phi\left(\gamma\left|H_{k}\right|^{2}\right)\right)\right)
\end{array}
$$

A comparação será segmentada em duas etapas. Primeiramente, a cutoff rate do sistema OFDM será comparada com a cutoff rate do DFE-SCCP. Em seguida, será realizada a comparação entre as cutoff rates dos sistemas LE-SCCP e OFDM. Começando pela comparação 
entre os sistemas OFDM e DFE-SCCP, introduzimos $\xi(x)=\tau\left(\phi^{-1}(x)\right)$, de modo que:

$$
\begin{aligned}
& \zeta_{O F D M}=\frac{1}{N} \sum_{k=0}^{N-1} \xi\left(\phi\left(\gamma\left|H_{k}\right|^{2}\right)\right) \\
& \zeta_{D F E}=\xi\left(\frac{1}{N} \sum_{k=0}^{N-1} \phi\left(\gamma\left|H_{k}\right|^{2}\right)\right)
\end{aligned}
$$

Definindo ainda, como feito no capítulo anterior, o mapeamento $y_{k}$ :

$$
y_{k}=\phi\left(\gamma\left|H_{k}\right|^{2}\right)=\log \left(1+\gamma\left|H_{k}\right|^{2}\right)
$$

as eqs. (5.31) e (5.32) podem ser expressas como:

$$
\begin{aligned}
& \zeta_{O F D M}=\frac{1}{N} \sum_{k=0}^{N-1} \xi\left(y_{k}\right) \\
& \zeta_{D F E}=\xi\left(\frac{1}{N} \sum_{k=0}^{N-1} y_{k}\right)
\end{aligned}
$$

Se a função $\xi(y)$ for convexa, a desigualdade de Jensen garante que $\xi\left(\frac{1}{N} \sum_{k=0}^{N-1} y_{k}\right) \leq$ $\frac{1}{N} \sum_{k=0}^{N-1} \xi\left(y_{k}\right)$. Portanto, a convexidade de $\xi(y)$ garante que $\zeta_{D F E}<\zeta_{O F D M}$ e, como consequência direta, $R_{0, D F E}>R_{0, O F D M}$.

A função $\xi(y)$ é convexa se e somente se sua segunda derivada:

$$
\frac{d^{2}}{d y^{2}} \xi(y)=\frac{\beta A_{l, m}}{2 R} \exp (y)\left(\frac{\beta A_{l, m}}{2 R} \exp (y)-1\right) \xi(y)
$$

for não negativa.

Como a função $\xi(y)$ e as constantes $\beta, A_{l, m}$ e $R$ são não-negativas, o sinal da segunda derivada de $\xi(y)$ é determinado pelo fator $c(y)=\frac{\beta A_{l, m}}{2 R} \exp (y)-1$.

Lembrando que a função $\xi(y)$ é calculada nos pontos definidos pelo mapeamento expresso na eq. (5.33), a função $c(y)$ assume a forma:

$$
\left.c(y)\right|_{\log \left(1+\gamma\left|H_{k}\right|^{2}\right)}=\gamma\left|H_{k}\right|^{2} \geq 0
$$

Sendo $c(y) \geq 0, \xi(y)$ é convexa. Dessa forma, pode-se dizer que a cutoff rate associada ao sistema DFE é sempre superior à cutoff rate do sistema OFDM. 
Nos resta comparar a cutoff rate do sistema OFDM com a cutoff rate do sistema LESCCP. Analogamente à comparação entre os sistemas OFDM e DFE-SCCP, definimos $\rho(x)=$ $\tau\left(\theta^{-1}(x)\right)$ :

$$
\begin{gathered}
\zeta_{O F D M}=\frac{1}{N} \sum_{k=0}^{N-1} \rho\left(\theta\left(\gamma\left|H_{k}\right|^{2}\right)\right) \\
\zeta_{L E}=\rho\left(\frac{1}{N} \sum_{k=0}^{N-1} \theta\left(\gamma\left|H_{k}\right|^{2}\right)\right)
\end{gathered}
$$

Definindo o mapeamento:

$$
x_{k}=\theta\left(\gamma\left|H_{k}\right|^{2}\right)=\frac{1}{1+\gamma\left|H_{k}\right|^{2}}
$$

as eqs. (5.38) e (5.39) podem ser expressas como:

$$
\begin{gathered}
\zeta_{O F D M}=\frac{1}{N} \sum_{k=0}^{N-1} \rho\left(x_{k}\right) \\
\zeta_{L E}=\rho\left(\frac{1}{N} \sum_{k=0}^{N-1} x_{k}\right)
\end{gathered}
$$

Assim como feito na comparação entre os sistemas OFDM e DFE-SCCP, serão exploradas as características de convexidade da função $\rho(x)$. Para tanto, é preciso analisar sua segunda derivada:

$$
\frac{d^{2}}{d x^{2}} \rho(x)=\frac{1}{x^{3}} \frac{\beta A_{l, m}}{2 R}\left(\frac{1}{x} \frac{\beta A_{l, m}}{2 R}-2\right) \rho(x)
$$

Apesar de não ser possível chegar a conclusões definitivas a respeito do sinal da segunda derivada de $\rho(x)$, pode-se lembrar que $\rho(x)$ é calculada em $x_{k}=\theta\left(\gamma\left|H_{k}\right|^{2}\right)=\frac{1}{1+\gamma\left|H_{k}\right|^{2}}$, e assim, pode-se calcular um limiar $\gamma_{m, L E}$, tal que $\gamma>\gamma_{m, L E} \Rightarrow R_{0, L E}>R_{0, O F D M}$. Esse valor de $\gamma_{m, L E}$ é dado por:

$$
\gamma_{m, L E}=\max _{k} \frac{1}{\left|H_{k}\right|^{2}}\left(\frac{4 R}{\beta A_{l, m}}-1\right)
$$

Para valores de SNR elevados, portanto, o desempenho do LE-SCCP tende a superar o OFDM, em termos de cutoff rate. É ainda importante notar que apesar de $\gamma>\gamma_{m, L E}$ garantir que $R_{0, L E}>R_{0, O F D M}$, a recíproca não é verdadeira. Em outras palavras, se $\gamma<\gamma_{m, L E}$, nada se pode afirmar a respeito da comparação entre a cutoff rate do sistema OFDM e a do sistema LE-SCCP. Lembrando ainda que $\beta$ decresce com a ordem da modulação, pode-se concluir 
que o valor limiar de SNR é crescente com a cardinalidade da modulação, i.e., a região em que o OFDM apresenta cutoff rate maior que o LE-SCCP aumenta.

A comparação entre as cutoff rates dos sistemas LE-SCCP e DFE-SCCP é imediata. Como, partindo da hipótese de que não há propagação de erros no DFE, a SNR do filtro linear é sempre menor do que a SNR do DFE, a eq. (5.15) garante que $R_{0, D F E}>R_{0, L E}$.

\subsubsection{Resultados}

A etapa seguinte consiste em analisar como a cutoff rate dos sistemas considerados se comporta em canais seletivos em frequência com desvanecimento por bloco. Para tanto, procede-se como feito em [25]: estipula-se uma taxa alvo e determina-se qual a probabilidade da cutoff rate ser menor do que a taxa de transmissão. Em analogia com a capacidade de canal, estima-se a probabilidade de haver outage.

Os cenários considerados consistem em modulações QPSK e 16-QAM em canal com desvanecimento Rayleigh e três coeficientes independentes:

$$
H(z)=h_{0}+h_{1} z^{-1}+h_{2} z^{-2}
$$

em que $h_{k} \sim \operatorname{eN}(0,1 / 3)$.

Em relação às taxas de codificação, foram adotadas as taxas $R=1 / 2,3 / 4,5 / 6$ e 7/8. As curvas de outage correspondentes estão mostradas nas Fig. 5.1 e 5.2.

Analisando a Fig. 5.1, percebe-se que à medida que a taxa de codificação aumenta, o OFDM fica mais susceptível a apresentar situações de outage. Note que para $R>1 / 2$, o desempenho do OFDM se mostra inferior ao do LE-SCCP, o que corrobora o resultado apresentado no capítulo 4.

Percebe-se também que, quando são utilizados códigos com taxas mais baixas, a diferença entre os sistemas tende a diminuir. Tal fato é condizente com as observações feitas em [16] de que o uso de codificação de canal é fundamental para se obter um bom desempenho no OFDM. Pelos resultados apresentados, se faz necessário o uso de códigos com rendimentos próximos de $R=1 / 2$ para que o desempenho do OFDM se aproxime do desempenho do LE-SCCP, na condição de canal escolhida.

No caso de modulação 16-QAM, analisando a Fig. 5.2, nota-se que para a taxa de codificação $R=1 / 2$, o desempenho do sistema OFDM é superior ao desempenho do sistema LE-SCCP. Enquanto que no caso QPSK, os sistemas LE-SCCP e OFDM apresentavam a mesma probabilidade de outage para $R=1 / 2$, no caso 16-QAM os sistemas apresentam a mesma probabilidade de outage para $R=3 / 4$. A perda de desempenho do LE-SCCP 

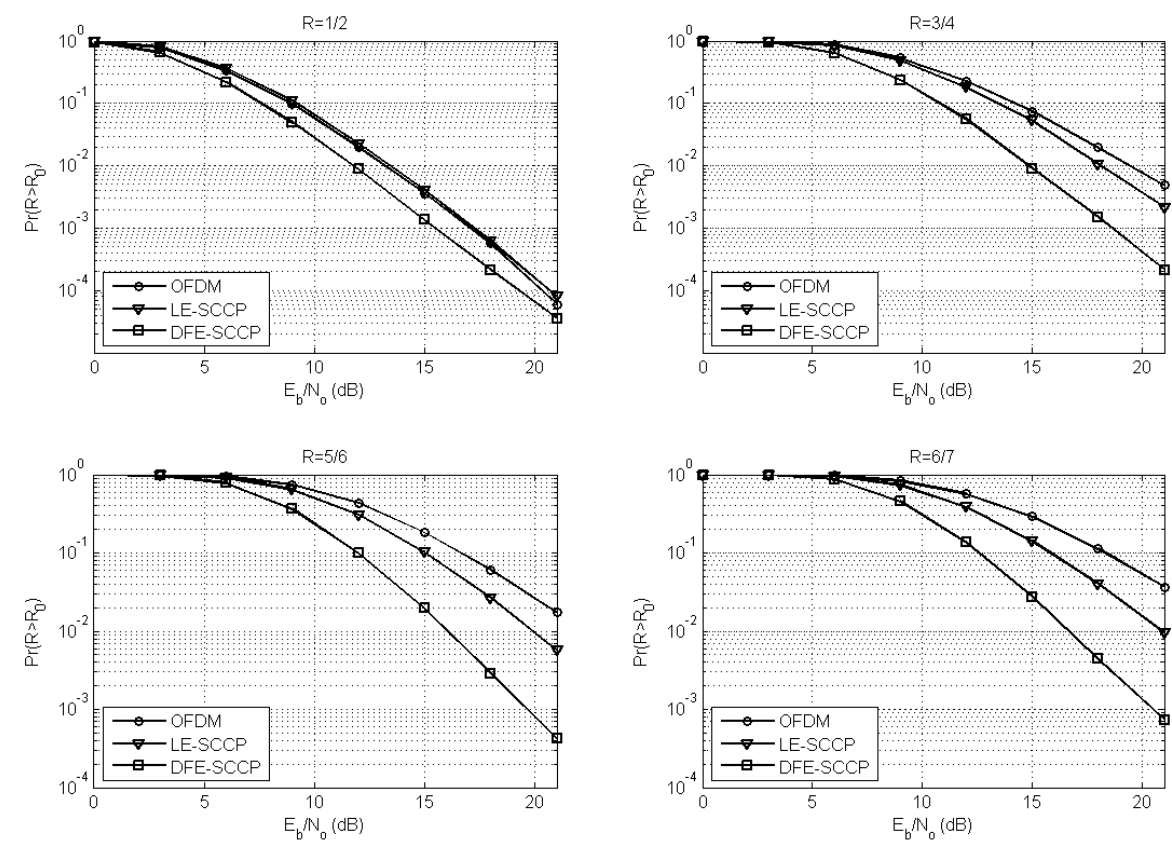

Figura 5.1: Probabilidade de transmissão acima da cutoff rate $-H(z)=h_{0}+h_{1} z^{-1}+h_{2} z^{-2}$, modulação QPSK.

neste caso corrobora a análise de cardinalidade apresentada no capítulo 4 . Contudo, o DFE-SCCP parece imune ao aumento na ordem da modulação, aumentando ainda mais a diferença de desempenho em relação ao OFDM, fato que contradiz os resultados do capítulo 4. Na verdade, como será visto no capítulo seguinte, os resultados fornecidos pela cutoff rate tendem a ser pessimistas em relação ao OFDM. De qualquer modo, a sensibilidade do OFDM em relação ao rendimento do código se mostra válida.

Uma outra questão que poderia ser posta é como sistemas de mesma eficiência espectral se comportam. Para tanto, serão comparadas as probabilidades de outage por cutoff rate para os sistemas em estudo em dois contextos distintos: modulação QPSK com taxa $R=1 / 2$ e modulação 16-QAM com $R=1 / 4$. Em ambas as situações, a eficiência espectral é fixada como $\eta=R \log _{2}(M)=1$. Os resultados estão indicados na Fig. 5.3. Note que tanto o sistema OFDM quanto o sistema SCCP apresentam melhor desempenho na situação em que, apesar de ser utilizada modulação de cardinalidade mais elevada, é utilizado um código mais eficiente. 

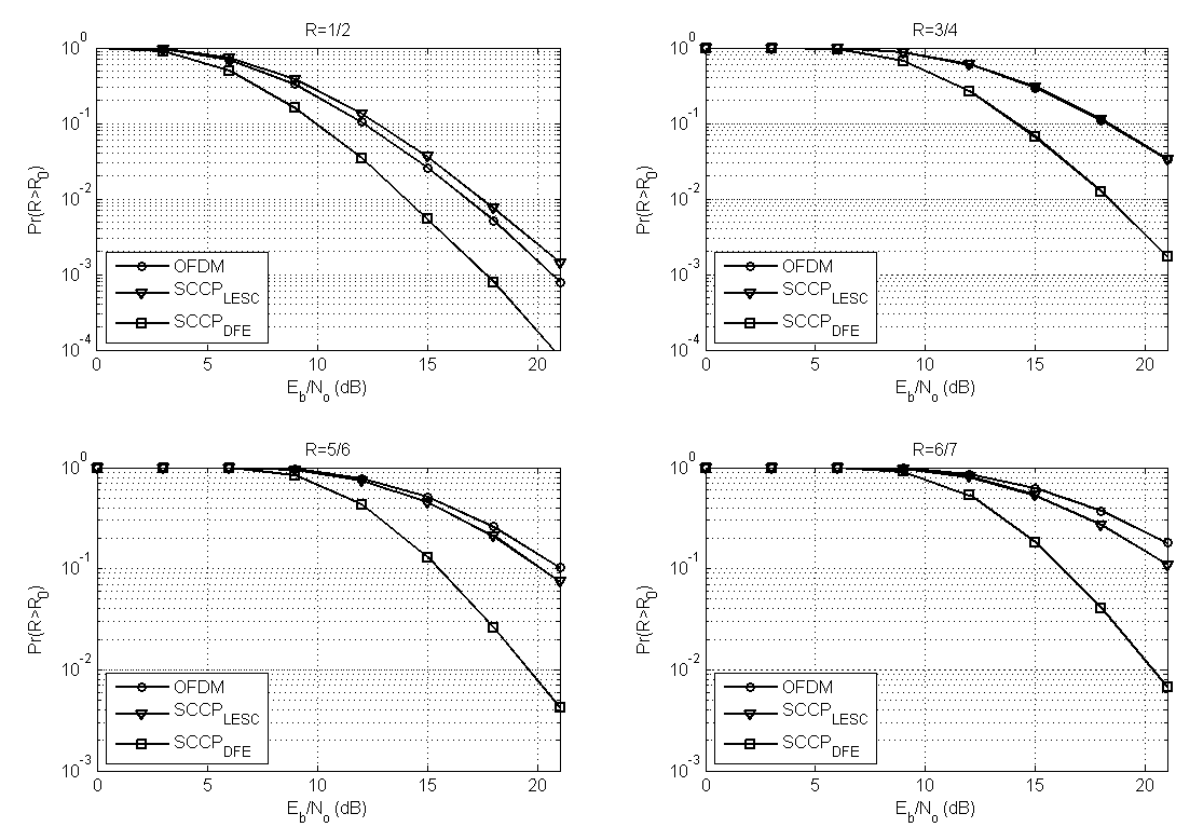

Figura 5.2: Probabilidade de transmissão acima da cutoff rate $-H(z)=h_{0}+h_{1} z^{-1}+h_{2} z^{-2}$, modulação 16-QAM.

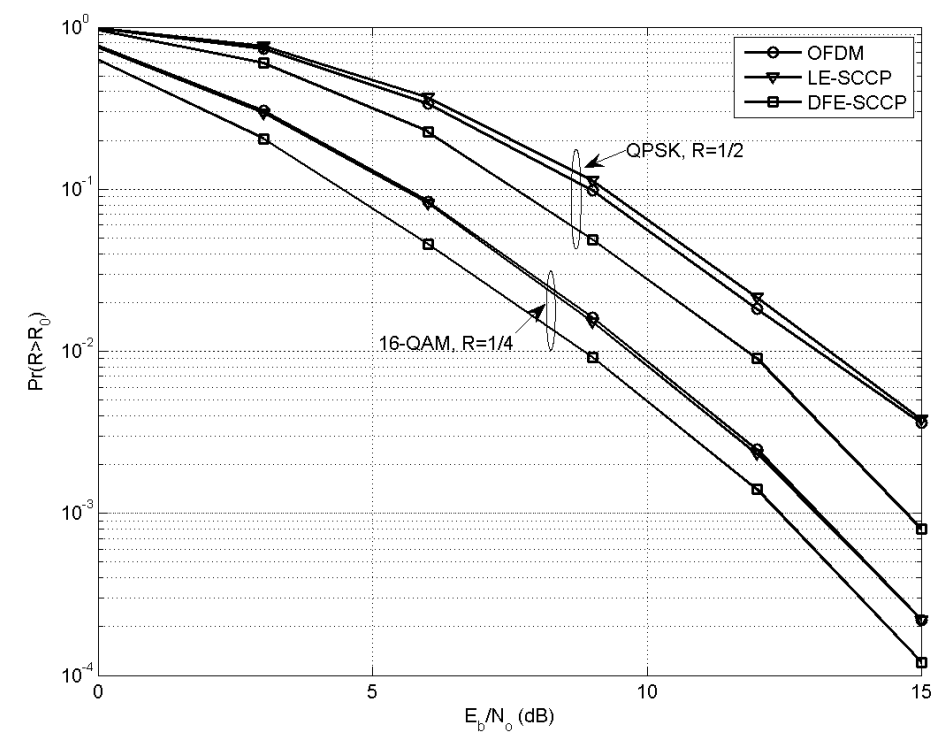

Figura 5.3: Probabilidade de transmissão acima da cutoff rate $-H(z)=h_{0}+h_{1} z^{-1}+h_{2} z^{-2}$, eficiência espectral fixada em $\eta=1$. 


\section{Sumário}

Neste capítulo a comparação entre os sistemas foi abordada do ponto de vista da codificação de canal.

Inicialmente, mostrou-se que na análise de capacidade de Shannon, os sistemas OFDM e DFE-SCCP apresentam o mesmo desempenho. Entretanto, a capacidade de Shannon parte da idealização que a transmissão se dá com símbolos de uma constelação gaussiana, i.e. uma constelação de ordem infinita. A fim de preencher essa lacuna, introduzimos a análise de cutoff rate. Nesse caso estendemos os resultados mostrados em [25] para o sistema DFESCCP e foi proposta uma análise, baseada em convexidade de funções, que pode ser aplicada a modulações M-QAM de qualquer cardinalidade.

No âmbito da cutoff rate, foi mostrado que o desempenho do sistema DFE-SCCP é sempre superior ao dos sistemas OFDM e LE-SCCP. Em relação aos sistemas LE-SCCP e OFDM, corroboramos a análise apresentada em [25], na qual é mostrado que a cutoff rate do sistema OFDM tende a se degradar em relação ao SCCP com o aumento da taxa de codificação.

Além disso, a extensão para outros níveis de modulação, nos permitiu perceber a degradação de desempenho do sistema LE-SCCP com a cardinalidade da modulação. Então, apesar dos resultados gerados no capítulo 4 terem sido obtidos a partir da premissa que a transmissão se dava sem o emprego de codificação de canal, os resultados providos pela análise de cutoff rate indicam que, no tocante à sensibilidade ao nível de modulação, as conclusões geradas no contexto sem codificação de canal também podem ser estendidas para o caso em que codificação é empregada.

O tratamento teórico apresentado nesse capítulo se fundamenta na premissa que são utilizados códigos de bloco de comprimento infinito com entrelaçamento ideal [25]. Na prática, não conseguimos tais condições. No capítulo seguinte, os sistemas serão comparados a partir do momento em que restrições de ordem práticas são impostas. 


\section{Capítulo 6}

\section{Efeitos do entrelaçador}

Um outro importante parâmetro de sistemas codificados, e que possui grande influência no desempenho, é a forma do entrelaçamento adotado. No capítulo anterior, apesar de se ter tratado da codificação de canal, a questão do entrelaçador foi deixada de lado. Neste capítulo será avaliado o efeito das configurações de entrelaçamento nos sistemas estudados.

Na maioria dos trabalhos envolvendo comparações entre OFDM e SCCP não é dada muita ênfase a esse assunto. Usualmente não se faz referência ao entrelaçador escolhido, ou não é dito qual o critério de escolha (e.g. [16] e [22]). Essa escolha, todavia, pode ser impactante no desempenho do sistema tal como observado em [56] no caso do entrelaçador modular.

A importância do entrelaçador de bits vem do fato de que os códigos são projetados a partir da premissa de que o sinal é submetido a um canal AWGN e, nessas condições, os erros ocorrem de maneira independente na saída do decodificador.

No nosso contexto são tratados canais seletivos em frequência e os sistemas OFDM e SCCP vão responder de maneiras distintas à seletividade do canal.

No caso do OFDM, as subportadoras fortemente atenuadas serão traduzidas em erros. Logo, com o objetivo de evitar rajadas de erros devemos projetar um entrelaçador de bits que consiga esparsar os erros da maneira mais eficaz possível. Sendo assim, o canal visto pelo decodificador pode se aproximar do canal AWGN.

Já no caso SCCP, a função do entrelaçador é quebrar a correlação do ruído no decodificador. Além disso, no caso do DFE-SCCP, o entrelaçador diminui a correlação da rajada de erros.

A fim de avaliar o impacto do entrelaçamento nos sistemas estudados, trataremos de três tipos de entrelaçadores diferentes: o entrelaçador regular, o modular e o aleatório. 


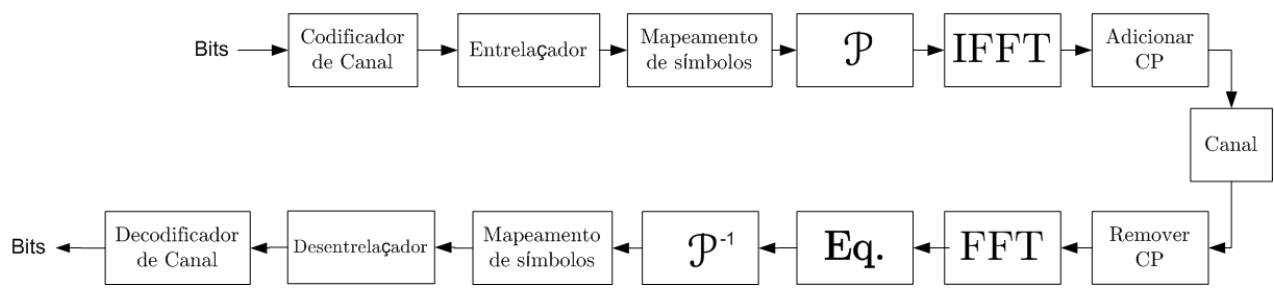

Figura 6.1: Modelo do sistema.

A análise, que será feita via simulação Monte Carlo, tratará de diversos cenários: canais seletivos em frequência, com desvanecimento Rayleigh e estático, diferentes taxas de codificação e várias modulações.

\subsection{Entrelaçamento}

As técnicas de coded modulation são implementadas através de uma das seguintes estruturas: BICM (Bit Interleaved Coded Modulation) [57] ou TCM (Trellis Coded Modulation), [58] [59].

Na nossa análise, será dada ênfase ao BICM. A escolha do BICM se deve ao fato de que podemos interpretar a transmissão OFDM, em canais seletivos em frequência, como uma transmissão em canal variante no tempo. Nessas condições, o BICM é uma estrutura mais adequada do que o TCM [57]. O esquema geral incluindo o BICM e o sistema apresentado no capítulo 2 está mostrado na Fig. 6.1.

O entrelaçamento nada mais é do que uma função de permutação aplicada a uma sequência de bits. Há várias formas de se definir a função de permutação, assim como detalhado em [60]. Analisaremos o entrelaçador regular, o entrelaçador modular apresentado em [56] e o entrelaçador aleatório do tipo S.

\subsubsection{Entrelaçamento regular}

O entrelaçador regular é a maneira clássica de entrelaçar bits. Para entrelaçar $N_{b}$ bits, deve ser definida uma matriz de entrelaçamento com $m$ linhas e $n=N_{b} / m$ colunas. O procedimento consiste em preencher a matriz por suas colunas e ler os bits através das linhas 
como ilustrado na expressão abaixo:

$$
\begin{gathered}
{\left[\begin{array}{c}
x_{0} \\
x_{1} \\
x_{2} \\
\vdots \\
x_{N_{b}-1}
\end{array}\right] \stackrel{\text { escrita }}{\longrightarrow}\left[\begin{array}{cccc}
x_{0} & x_{m} & \cdots & x_{(n-1) m} \\
x_{1} & x_{m+1} & \cdots & x_{(n-1) m+1} \\
\vdots & \vdots & & \vdots \\
x_{m-1} & x_{2 m-1} & \cdots & x_{m n-1}
\end{array}\right]} \\
\\
{\left[\begin{array}{c}
\downarrow \\
x_{0} \\
x_{m} \\
x_{2 m} \\
\vdots \\
x_{N_{b}-1}
\end{array}\right]}
\end{gathered}
$$

Note que procedendo dessa maneira, garante-se um espaçamento mínimo de $n$ bits entre dois bits consecutivos na sequência original. Além disso, dado que o índice do primeiro bit é $k=0$, observe que o índice do segundo bit entrelaçado é $k=m$. Para as discussões subsequentes, é importante ter claro o significado dos parâmetros $m$ e $n$ :

1. Parâmetro $m$ - distância existente, na sequência original, entre dois bits consecutivos na sequência entrelaçada

2. Parâmetro $n$ - separação mínima, na sequência entrelaçada, imposta entre dois bits consecutivos na sequência original

A distância entre dois bits consecutivos da sequência original difere de $n$ bits na sequência entrelaçada, apenas nos casos em que tais bits consecutivos têm índices da forma $\mathrm{km}-1 \mathrm{e} k m$, em que $k$ é qualquer natural positivo menor do que $n$. Observe que tais bits correspondem ao último bit da $k$-ésima coluna e ao primeiro bit da $(k+1)$-ésima coluna. Nessa situação, na sequência entrelaçada, a distância entre eles é dada por $N_{b}-n$.

\subsubsection{Entrelaçamento modular}

Uma outra forma de entrelaçar os bits do vetor $\mathbf{x}$ é através de um entrelaçador modular [56]. Denotando por $\mathbf{k}$ o conjunto de índices do vetor $\mathbf{x}$, o entrelaçador modular é implementado aplicando a seguinte função de permutação à sequência de índices original:

$$
\Pi(\mathbf{k}) \equiv N_{1} \mathbf{k} \quad \bmod \left(N_{b}\right)
$$


A fim de garantir a injetividade do mapeamento, $N_{1}$ precisa ser escolhido de modo que $\operatorname{MDC}\left(N_{1}, N_{b}\right)=1$.

O mapeamento inverso é dado por:

$$
\Pi^{-1}(\mathbf{x}) \equiv N_{1}^{-1} \mathbf{x} \quad \bmod \left(N_{b}\right)
$$

Na expressão acima, $N_{1}^{-1}$ é um inteiro é tal que:

$$
N_{1} N_{1}^{-1} \equiv 1 \quad \bmod \left(N_{b}\right)
$$

Veja que a condição $\operatorname{MDC}\left(N_{1}, N_{b}\right)=1$ garante a existência e a unicidade de $N_{1}^{-1}$ [61]. Em geral, como o número de bits por bloco é uma potência de 2, qualquer número natural ímpar satisfaz a condição de injetividade do mapeamento e pode ser definido como parâmetro de entrelaçamento. Além disso, é interessante notar que o parâmetro $N_{1}$ do entrelaçador modular desempenha função semelhante à do parâmetro $m$ do entrelaçador regular.

No entrelaçador modular, o segundo bit da sequência entrelaçada corresponde ao bit de índice $N_{1}$ da sequência original. Assim como no regular, o segundo bit corresponde ao bit de índice $m$ na sequência original.

Já o papel do parâmetro $N_{1}^{-1}$ é semelhante ao do parâmetro $n$ do entrelaçamento regular. Observe que o índice $k$ que será mapeado no segundo elemento da sequência entrelaçada é dado por:

$$
N_{1} k \equiv 1 \bmod \left(N_{b}\right) \Longleftrightarrow k \equiv N_{1}^{-1} \bmod \left(N_{b}\right)
$$

Assim, o espaçamento entre os dois primeiros bits da sequência original é de $N_{1}^{-1}$ na sequência entrelaçada. Como veremos no exemplo mostrado mais adiante, a circularidade do mapeamento faz com que o espaçamento entre dois bits consecutivos quaisquer oscile entre $N_{1}^{-1}$ e $N_{b}-N_{1}^{-1}$.

Apesar das semelhanças entre as duas classes de entrelaçadores apresentadas, é necessário destacar algumas diferenças fundamentais entre elas. Primeiramente, note que no entrelaçador regular, os parâmetros $n$ e $m$ devem satisfazer a condição $N_{b}=n m$. Em outras palavras, $\operatorname{MDC}\left(N_{b}, m\right)=m$ e $\operatorname{MDC}\left(N_{b}, n\right)=n$. Já no entrelaçador modular, é necessário que $\operatorname{MDC}\left(N_{1}, N_{b}\right)=1$ para que o mapeamento seja definido.

Uma segunda diferença consiste no fato do entrelaçador regular manter as posições do primeiro e do último bit da sequência original, enquanto que o entrelaçador modular mantém apenas a posição do primeiro bit. O último bit entrelaçado corresponde a: 
(a)

\begin{tabular}{|l|l|l|l|l|l|l|l|l|l|l|l|l|l|l|l|}
\hline$X_{0}$ & $X_{1}$ & $X_{2}$ & $X_{3}$ & $X_{4}$ & $X_{5}$ & $X_{6}$ & $X_{7}$ & $X_{8}$ & $X_{9}$ & $X_{10}$ & $X_{11}$ & $X_{12}$ & $X_{13}$ & $X_{14}$ & $X_{15}$ \\
\hline
\end{tabular}

(b)

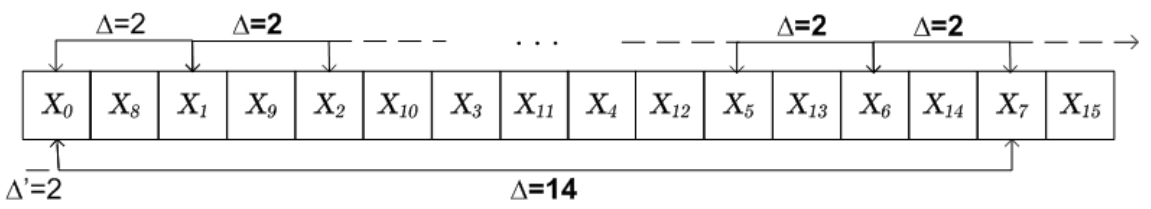

(c)

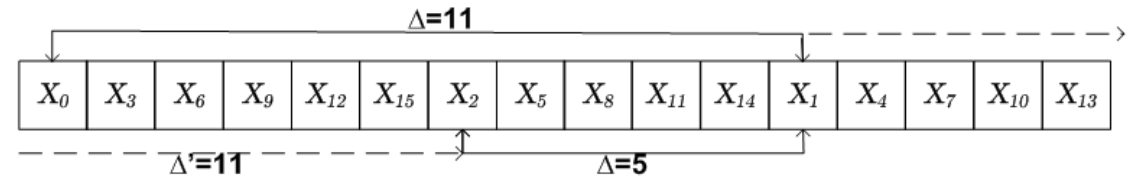

Figura 6.2: Exemplo de entrelaçamento de uma sequência com $N_{b}=16$ bits. (a) sequência original, (b) sequência entrelaçada com um entrelaçador regular com $m=8$ linhas e (c) sequência entrelaçada com entrelaçador modular com parâmetro $N_{1}=3$.

$$
\begin{aligned}
\Pi\left(N_{b}-1\right) & \equiv N_{1}\left(N_{b}-1\right) \quad \bmod \left(N_{b}\right) \\
& \equiv-N_{1} \quad \bmod \left(N_{b}\right) \\
& \equiv N_{b}-N_{1} \quad \bmod \left(N_{b}\right)
\end{aligned}
$$

Já o último bit da sequência original é mapeado no índice $k$ da sequência entrelaçada, com $k$ satisfazendo:

$$
\begin{aligned}
k N_{1} & \equiv N_{b}-1 \quad \bmod \left(N_{b}\right) \\
k & \equiv\left(N_{1}^{-1}\right)\left(N_{b}-1\right) \quad \bmod \left(N_{b}\right) \\
k & \equiv N_{b}-N_{1}^{-1} \quad \bmod \left(N_{b}\right)
\end{aligned}
$$

Para ilustrar de forma mais clara tais diferenças, considere como exemplo o entrelaçamento de um bloco de $N_{b}=16$ bits utilizando um entrelaçador regular e um entrelaçador modular. Para o entrelaçador regular, fixa-se $m=8$ e, consequentemente, $n=2$. Já para o entrelaçador modular, fixa-se $N_{1}=3$. O inverso multiplicativo de $N_{1}$ na aritmética módulo $N_{b}=16$ é $N_{1}^{-1}=11$. A Fig. 6.2 mostra a sequência de bits original e as sequências geradas com os entrelaçadores regular e modular.

Note que, de fato, o mapeamento modular impõe uma distância de $N_{1}^{-1}=11$ bits entre os dois primeiros bits da sequência original. A circularidade do mapeamento faz com que a distância entre o segundo e o terceiro bits, $X_{1}$ e $X_{2}$, da sequência original seja $\Delta=N_{b}-N_{1}^{-1}=$ 5. Já a distância entre os bits $X_{2}$ e $X_{3}$ volta a ser dada por $N_{1}^{-1}=11$. Desse modo, percebe- 
se que essa distância sempre oscila entre $N_{1}^{-1}=11$ e $N_{b}-N_{1}^{-1}=5$. A oscilação se dá obedecendo a um padrão determinado pela relação entre $N_{1}^{-1}$ e $N_{b}$. Ainda observa-se que o último bit da sequência original, $X_{15}$, é realmente mapeado no índice $k=N_{b}-N_{1}^{-1}=5$ na sequência entrelaçada. E o último bit da sequência entrelaçada corresponde ao bit com índice $k=N_{b}-N_{1}=13$ da sequência original.

Em relação ao entrelaçamento regular, percebe-se que, de fato, a posição do último bit não se altera no entrelaçamento e com exceção dos bits consecutivos $X_{7}$ e $X_{8}$, a distância entre quaisquer dois pares de bits consecutivos é dada por $m=8$, de acordo com o discutido na subseção anterior.

\subsubsection{Entrelaçamento aleatório}

Até então, discutimos duas classes de entrelaçamento com características bem específicas: os entrelaçadores regulares e modulares. Nos restringindo a tais classes de entrelaçamento, é possível representar apenas uma pequena fração do total de entrelaçadores possíveis para um bloco de $N_{b}$ bits. Em particular para o entrelaçador regular, denotando o número de divisores de $N_{b}$ por $D\left(N_{b}\right)$, é possível definir $D\left(N_{b}\right)$ entrelaçadores regulares distintos. Se $N_{b}$ for uma potência de $2, D\left(N_{b}\right)=\log _{2}\left(N_{b}\right)$. Em relação aos entrelaçadores modulares, podemos definir $\varphi\left(N_{b}\right)$ diferentes, em que $\varphi\left(N_{b}\right)$ é a função de Euler, i.e. o número de naturais $n \leq N_{b}$ tais que $\operatorname{MDC}\left(N_{b}, n\right)=1$. Ainda se $N_{b}$ potência for $2, \varphi\left(N_{b}\right)=N_{b} / 2$. Desse modo, a união das duas classes de entrelaçadores apresentadas consegue gerar $D\left(N_{b}\right)+\varphi\left(N_{b}\right)$ entrelaçadores distintos. Entretanto, sabemos que é possível definir $N_{b}$ ! permutações diferentes a partir de uma sequência de $N_{b}$ elementos. Há, portanto, $N_{b}$ ! $-D\left(N_{b}\right)-\phi\left(N_{b}\right)$ permutações não contabilizadas levando-se em conta as duas formas de entrelaçamento que já foram analisadas.

Com o objetivo de também cobrir os entrelaçadores que não se enquadram em nenhuma dos dois formatos, é definida uma terceira classe de entrelaçadores. Qualquer permutação aleatória dos bits da sequência original é elemento dessa classe. Os entrelaçadores pertencentes a essa família serão denotados por entrelaçadores aleatórios.

Impondo algumas restrições à função de permutação, é definido o Entrelaçador-S. De modo geral, o mapaemento dos índices nesses entrelaçadores deve ser tal que garanta uma distância mínima $d_{\min }=S$ entre quaisquer $S$ bits consecutivos [62].

O mapeamento aleatório convencional, portanto, é o próprio entrelaçador-S com o parâmetro $\mathrm{S}=1$. 


\subsection{Canal seletivo em frequência e estático}

No capítulo 1, comentou-se que, em canais com desvanecimento profundo, não é possível atingir valores de BER aceitáveis com o OFDM sem código.

A pergunta que surge é: a codificação de canal é suficiente para suprir as limitações do OFDM em canais com desvanecimento profundo?

Para responder a esse questionamento, serão considerados alguns canais estáticos e será analisado se é sempre possível determinar um padrão de entrelaçamento que leve o sistema OFDM a apresentar desempenho comparável com o sistema DFE-SCCP.

Os resultados mostrados nessa seção foram gerados utilizando o código convolucional $\left[\begin{array}{ll}15 & 17\end{array}\right]_{\text {octal }}$.

\subsubsection{Efeito do entrelaçamento}

A princípio, será considerarado o canal Proakis B [52], cuja função de transferência é dada por:

$$
H(z)=0,407+0,815 z^{-1}+0,407 z^{-2}
$$

Para esse canal, a sensibilidade às escolhas de parâmetros de cada uma das classes foi examinada isoladamente. A análise é feita fixando a relação $E_{b} / N_{o}$ e variando os parâmetros de entrelaçamento.

\section{Entrelaçamento Regular}

A análise do entrelaçador regular pode ser feita variando o número de linhas do entrelaçador e analisando o desempenho para cada uma das configurações. Com a relação $E_{b} / N_{o}$ fixada determinou-se a BER e taxa de erro de bloco (BLER, do inglês, block error rate) para os sistemas estudados nas diferentes configurações de entrelaçamento. Os resultados estão mostrados na Fig. 6.3.

Note que, no caso OFDM, a diferença de desempenho obtida com a melhor e a pior escolha de entrelaçador é muito pronunciada. Na melhor condição, o desempenho do OFDM é equivalente ao desempenho do DFE-SCCP perfeito. Já nas condições pouco favoráveis, o OFDM apresenta desempenho inferior ao do DFE-SCCP e ao do LE-SCCP. Ainda, podese notar uma enorme diferença entre os desempenhos do DFE e do DFE perfeito. Mais a frente, mostraremos que essa lacuna pode ser compensada utilizando-se técnicas de redução de propagação de erros. 


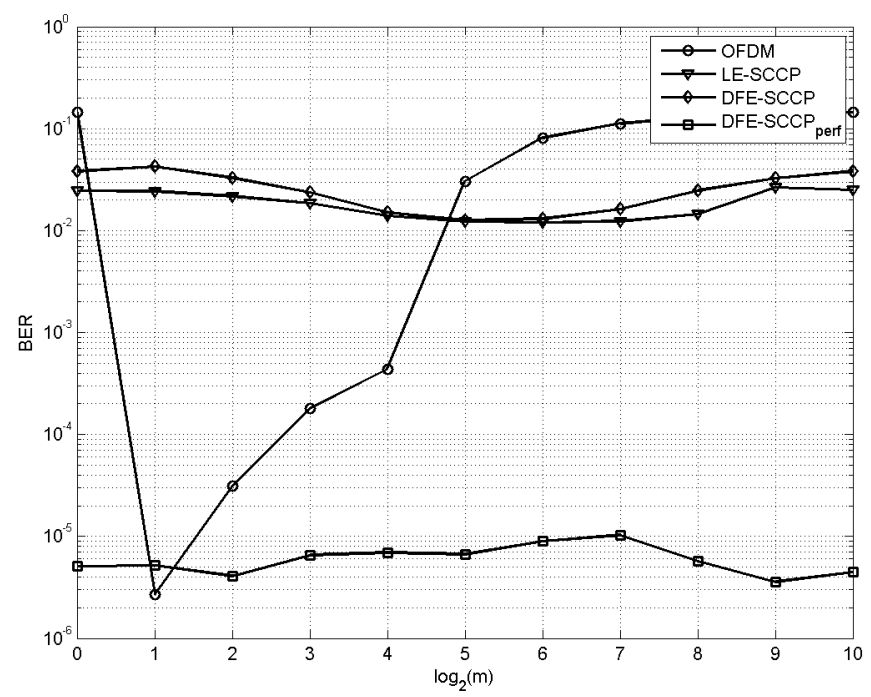

Figura 6.3: BER para entrelaçadores regulares com diferentes números de linhas para o canal Proakis $\mathrm{B}, E_{b} / N_{o}=8 \mathrm{~dB}$.

A fim de explicar a questão da degradação no OFDM, será analisada a magnitude da resposta em frequência deste canal.

Primeiramente, deve-se ter em mente que são transmitidos símbolos OFDM com $N=512$ subportadoras e modulação QPSK, de modo que em cada símbolo OFDM tem-se $N_{b}=2 N=$ 1024 bits transmitidos. Então, pode-se definir a seguinte função:

$$
G\left(k^{\prime}\right)=H\left(\left\lfloor\frac{k^{\prime}}{2}\right\rfloor\right), \quad k^{\prime}=0, \cdots, 2 N-1
$$

em que o operador $f(a)=\lfloor a\rfloor$ representa a função piso, i.e., $f$ mapeia a no menor inteiro igual ou maior do que $a$.

Considerando que os bits de um dado símbolo apresentam a mesma SNR na entrada do decodificador, temos que a SNR do $k^{\prime}$-ésimo bit codificado no bloco, sem entrelaçamento, é proporcional à magnitude da função $G\left(k^{\prime}\right)$ representada na Fig. 6.4. Para simplificar a notação, denota-se a SNR do $k^{\prime}$-ésimo bit codificado por $\gamma_{k^{\prime}}$.

Observando a Fig. 6.4, observa-se que os bits alocados em componentes com frequências mais altas estão em condições desfavoráveis de $\gamma_{k^{\prime}}$. Além disso, esses bits estão próximos uns dos outros, fato que se reflete como uma rajada de erros na saída do decodificador.

Agora, será determinado o entrelaçador regular de bits ideal, no caso OFDM, para evitar rajadas de erros nesse tipo de canal.

A partir do descrito na subseção 6.1, podemos afirmar que se for utilizado um entrelaçador 


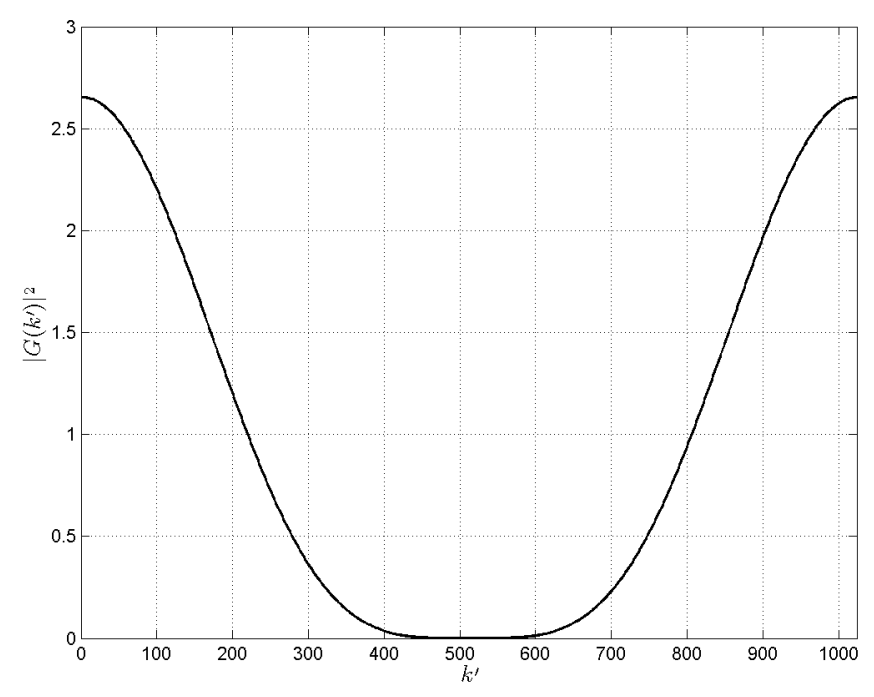

Figura 6.4: $\left|G\left(k^{\prime}\right)\right|^{2}$ para o canal Proakis B.

regular com $m$ linhas, a $\gamma_{k^{\prime}}$ em cada bit será dada por:

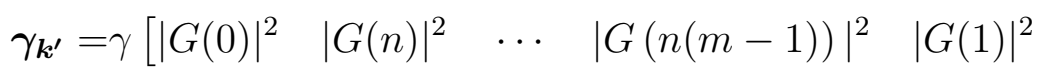

$$
\begin{aligned}
& \left.|G(1+m)|^{2} \cdots \quad|G(1+n(m-1))|^{2} \quad|G(n-1)|^{2} \quad \cdots \quad|G(m n-1)|^{2}\right]
\end{aligned}
$$

Para combater as rajadas de erros, os bits com baixa SNR devem estar intercalados com os bits de alta SNR. Como os bits de alta SNR estão na região de baixa frequência e as de baixa SNR estão na região de alta frequência, a eq. (6.9) indica que a escolha ótima de $n$ (número de colunas da matriz de entrelaçamento) deve estar em torno de $N$. Apesar de estarem sendo considerados blocos com $N=512$ subportadoras nas simulações, a fim de facilitar a visualização das subportadoras, será assumido que o sistema será implementado com blocos com $N=32$ subportadoras. A Fig. 6.5 mostra os canais equivalentes na entrada do decodificador para entrelaçadores com diferentes números de linhas. Pode-se notar que, à medida que o número de linhas do entrelaçador diminui, os erros vão ficando mais esparsos, melhorando o desempenho do código. Essa conclusão condiz com os resultados mostrados na Fig. 6.3.

\section{Entrelaçamento Modular}

Assim como feito para o entrelaçador regular, para uma $E_{b} / N_{o}$ fixada, as possíveis configurações de entrelaçamento serão testadas com o objetivo de avaliar a sensibilidade dos sistemas à escolha do parâmetro de entrelaçamento. 

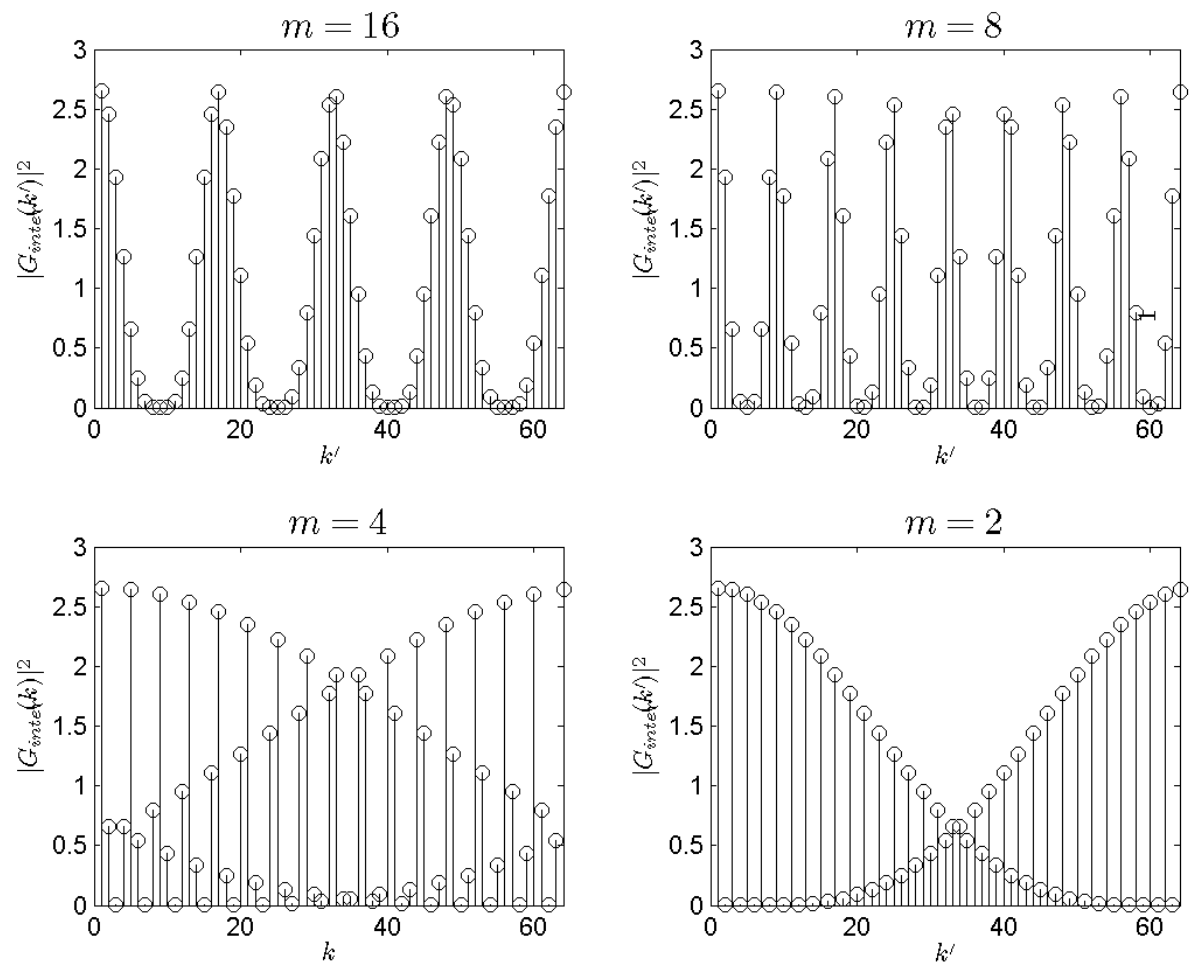

Figura 6.5: $\gamma_{k^{\prime}}$ 's nas subportadoras OFDM quando os bits são entrelaçados com um entrelaçador de $m$ linhas, canal Proakis B.

A Fig. 6.6 mostra que o sistema OFDM é também extremamente sensível à escolha do parâmetro de entrelaçamento modular. Como já foi discutido anteriormente, os entrelaçadores regular e modular guardam muitas características semelhantes. Portanto, a dependência do desempenho do sistema OFDM com a configuração de entrelaçamento regular já apontava que tal dependência também deveria ser notada no entrelaçador modular.

No caso do entrelaçador regular, foi mostrado que o desempenho do sistema OFDM era maximizado para $m=2$, i.e., $n=512$. Mas já foi mostrado que o parâmetro $N_{1}^{-1}$ do entrelaçador modular desempenha função semelhante a do parâmetro $n$ do entrelaçador regular. Portanto, assim como mostra a Fig. 6.6, o entrelaçador modular deve apresentar melhor desempenho para parâmetros $N_{1}^{-1}$ em torno de 512. Analisando os resultados na Fig. 6.6, $N_{1}^{-1}=519$ foi definido como parâmetro ótimo de entrelaçamento, i.e. o parâmetro de entrelaçamento que leva à menor BER. 


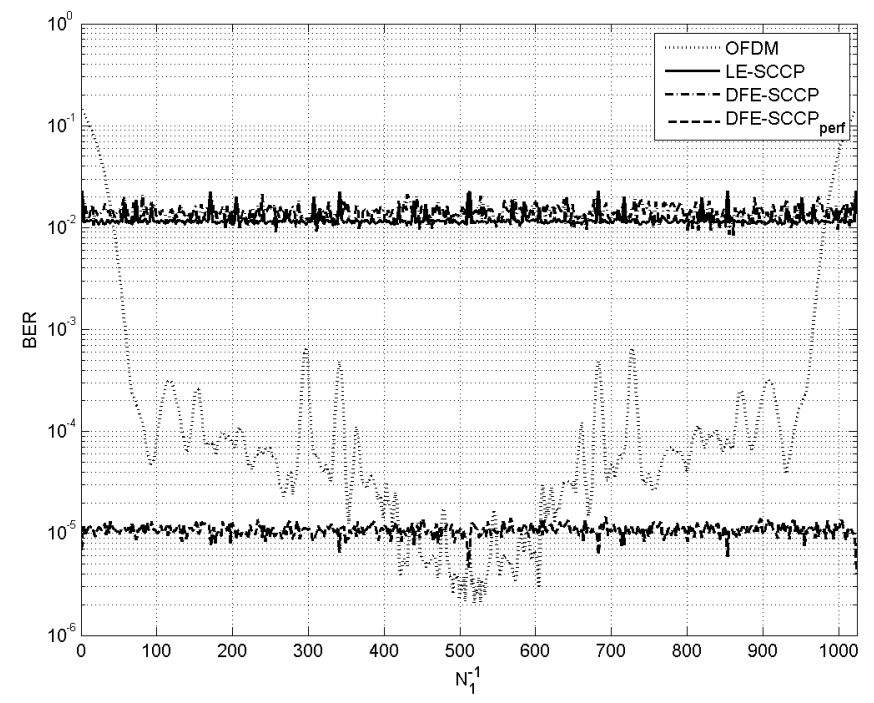

Figura 6.6: BER para entrelaçadores modulares com diferentes parâmetros $N_{1}^{-1}$ para o canal Proakis B, $E_{b} / N_{o}=8 \mathrm{~dB}$.

\section{Entrelaçamento Aleatório}

A partir das análises mostradas para os entrelaçadores modular e regular, nota-se a grande sensibilidade do OFDM à configuração de tais entrelaçadores. Sendo assim, é razoável supor que o desempenho do entrelaçador de características aleatórias seja inferior às melhores configurações de entrelaçamento regular e modular, uma vez que é altamente improvável que uma realização aleatória de entrelaçamento coincida com uma das configurações especiais de entrelaçamento que levam o OFDM a apresentar seu melhor desempenho.

A fim de avaliar a sensibilidade dos sistemas em relação aos entrelaçadores do tipo Saleatório, o procedimento será o mesmo já realizado para os entrelaçadores regular e modular. Para $E_{b} / N_{o}=8 \mathrm{~dB}$ fixada, através de simulação Monte Carlo, determina-se a BER para diferentes valores de parâmetro $S$.

É importante notar, entretanto, que fixado o parâmetro $S$, há um número proibitivamente grande de possíveis escolhas de entrelaçadores (no caso de $S=1$, há $N_{b}$ ! possibilidades). Dessa forma, não é factível simular todos os entrelaçadores e selecionar aquele que apresenta o melhor desempenho. Diante dessa dificuldade, para um dado parâmetro $S$, foram gerados vinte e cinco entrelaçadores e foi selecionado aquele que apresentava a menor BER.

A Fig. 6.7 mostra a BER para diferentes configurações de entrelaçamento aleatório. Note que nos sistemas SCCP, o desempenho obtido com o entrelaçador S-aleatório não é diferente daquele já obtido com os outros entrelaçadores. Porém, quando o sistema OFDM é considerado, há uma grande perda de desempenho em relação às melhores configurações 


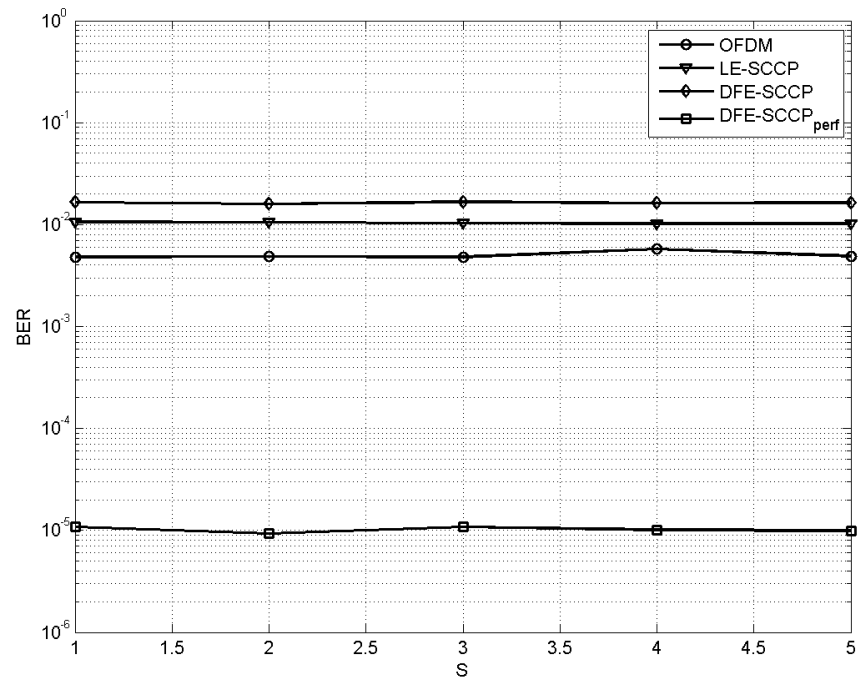

Figura 6.7: BER para entrelaçadores do tipo S-aleatório em função do parâmetro $S$ para o canal Proakis B, $E_{b} / N_{o}=8 \mathrm{~dB}$.

de entrelaçamento dos demais tipos de entrelaçadores.

Dado que a melhor configuração de entrelaçamento foi obtida para cada tipo de entrelaçador, a próxima etapa da análise consistirá na comparação entre os entrelaçadores com os parâmetros ótimos. Os resultados obtidos estão mostrados na Fig. 6.8. De acordo com o resultado indicado, a escolha ótima de entrelaçamento OFDM é o entrelaçador modular com parâmetro $N_{1}^{-1}=519 \Leftrightarrow N_{1}=915$.

Os resultados mostrados na Fig. 6.8 ainda permitem inferir que a escolha ótima de entrelaçamento é também função da SNR.

Uma outra questão importante a ser analisada é a robustez dessa solução em outros canais com desvanecimento profundo. Para avaliar esse ponto, serão considerados canais com função de transferência dada por:

$$
H(z)=\left(1-e^{j \theta} z^{-1}\right)\left(1-e^{-j \theta} z^{-1}\right) \quad 0 \leq \theta \leq \pi
$$

Os canais com função de transferência da forma da eq. (6.10) têm zeros no círculo unitário, gerando situações de difícil equalização.

A Fig. 6.9 mostra que para canais do tipo passa-baixa ou passa-alta, os entrelaçadores regular e modular que otimizam o desempenho do OFDM no canal Proakis B, também geram bons resultados para esses canais. Entretanto, se o canal for do tipo passa-faixa, o 


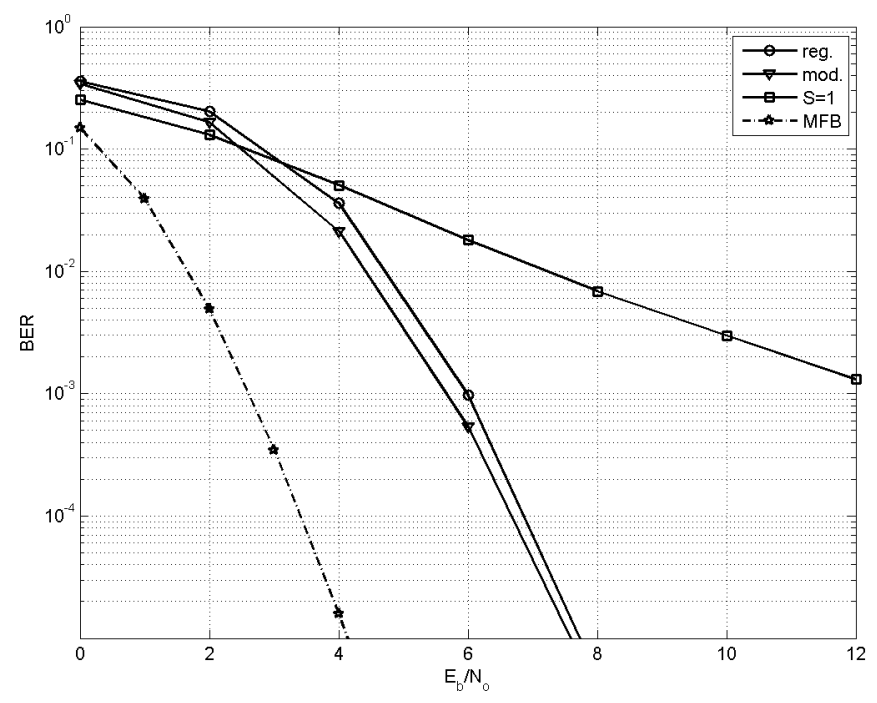

Figura 6.8: Comparação de desempenho do OFDM com diferentes tipos de entrelaçadores no canal Proakis B. Para o regular, $m=2$ e para o modular com $N_{1}=915$.

desempenho do sistema OFDM com os entrelaçadores regular e modular será extremamente degradado.

\subsubsection{Comparação de desempenho}

Dado que o efeito do entrelaçador já foi analisado e a configuração de entrelaçamento que leva ao melhor desempenho do sistema OFDM é conhecida, os sistemas OFDM, LE-SCCP, DFE-SCCP e DFE-SCCP perfeito serão comparados em termos de BER.

A fim de minimizar o efeito de propagação de erros, no caso do sistema DFE-SCCP, iremos empregar a técnica descrita em [63]. Nela, o DFE utiliza como realimentação decisões provenientes do caminho sobrevivente de um decodificador de canal para TCM. Os resultados estão mostrados na Fig. 6.10. Nela nota-se que a técnica de minimização de propagação de erros permite se aproximar consideravelmente do DFE perfeito. Ainda percebe-se que o OFDM, quando bem entreleçado, exibe desempenho que pode ser melhor que o do DFE perfeito. Além das curvas de BER dos sistemas, a Fig. 6.10 mostra a curva de matched filter bound (MFB) [53] que é um limitante fundamental do desempenho do sistema. A curva mostra o desempenho de um sistema capaz de maximizar a relação sinal ruído sem introduzir a interferência intersimbólica característica de um filtro casado.

Cabe a questão se sempre é possível selecionar entrelaçadores que levem o sistema OFDM a apresentar desempenho superior ao sistema DFE-SCCP perfeito ou se tal comportamento 


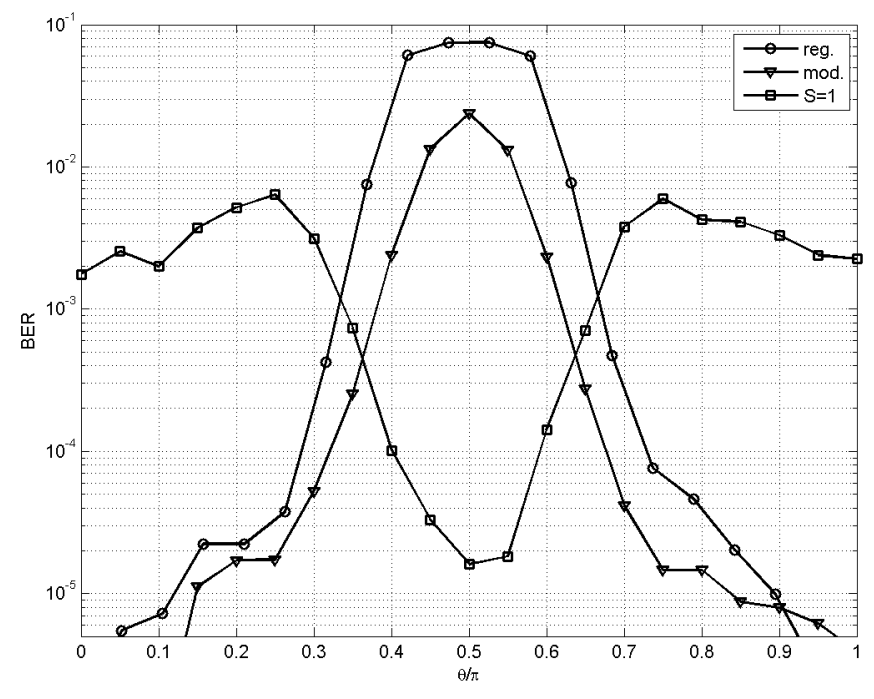

Figura 6.9: Comparação de desempenho para sistemas OFDM submetidos a canais com função de transferência dada pela eq. (6.10). Entrelaçador regular com $m=2$ linhas e entrelaçador modular com $N_{1}=915$.

é restrito a uma classe de canais.

Visando responder a tal pergunta, analisou-se o caso de transmissão no canal Proakis C [52], cuja função de transferência é dada por:

$$
H(z)=0,227+0,460 z^{-1}+0,688 z^{-2}+0,460 z^{-3}+0,227 z^{-4}
$$

De modo semelhante ao realizado no caso do Proakis B, a função $G\left(k^{\prime}\right)$ do canal será analisada com o objetivo de encontrar a melhor condição de entrelaçamento para o OFDM. Esta função é mostrada na Fig. 6.11.

Analisando a Fig. 6.11, percebe-se que a faixa de frequências rejeitada por este canal é maior do que no caso do Proakis B. Esse fato dificulta o estabelecimento de uma função de entrelaçamento que consiga mitigar as rajadas de erros no decodificador.

Novamente, da mesma forma realizada no canal Proakis B, procurou-se a melhor configuração de entrelaçador OFDM para o canal Proakis C e o desempenho foi comparado com o do DFE-SCCP. Os resultados estão mostrados na Fig. 6.12. Note que o desempenho do DFE-SCCP é bem superior em relação ao OFDM, diferindo significativamente do caso anterior. Portanto, não há como generalizar o comportamento dos sistemas, em especial, o do OFDM. 


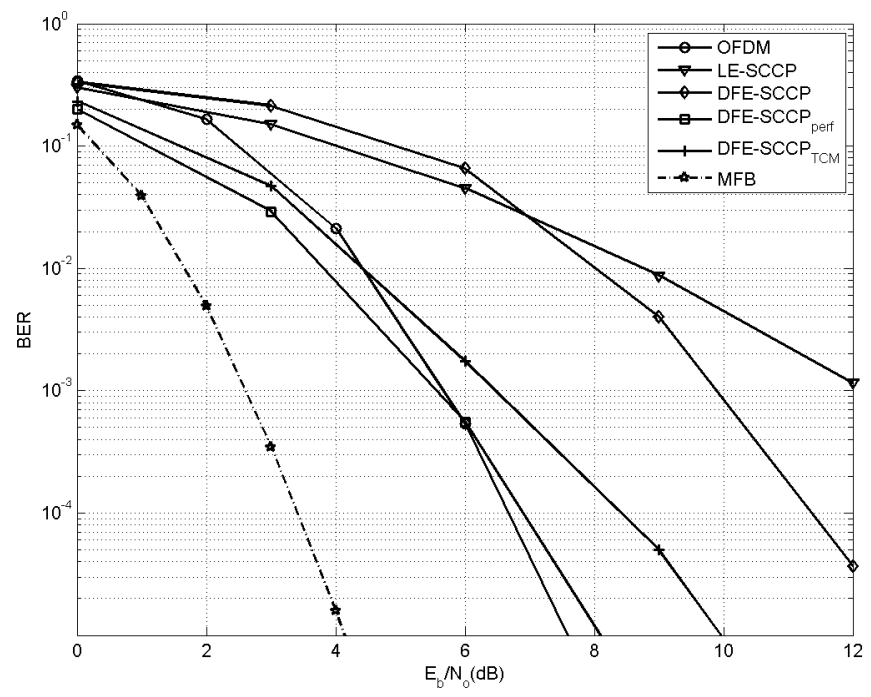

Figura 6.10: Comparação de desempenho, QPSK, canal Proakis B. OFDM implementado com entrelaçamento modular com $N_{1}=915$ e SCCP com entrelaçador regular com $m=32$ linhas.

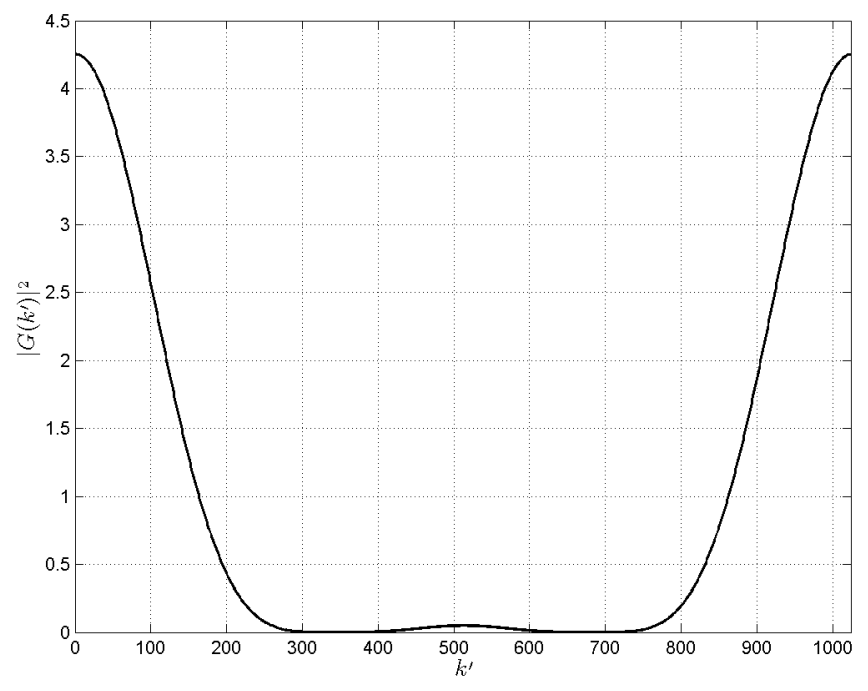

Figura 6.11: $\left|G\left(k^{\prime}\right)\right|^{2}$ para o canal Proakis C.

\subsection{Canais seletivos em frequência com desvanecimento por blocos}

Na seção anterior, os sistemas foram comparados em situações nas quais a transmissão ocorria em canais estáticos com desvanecimento profundo. Esse tipo de abordagem é im- 


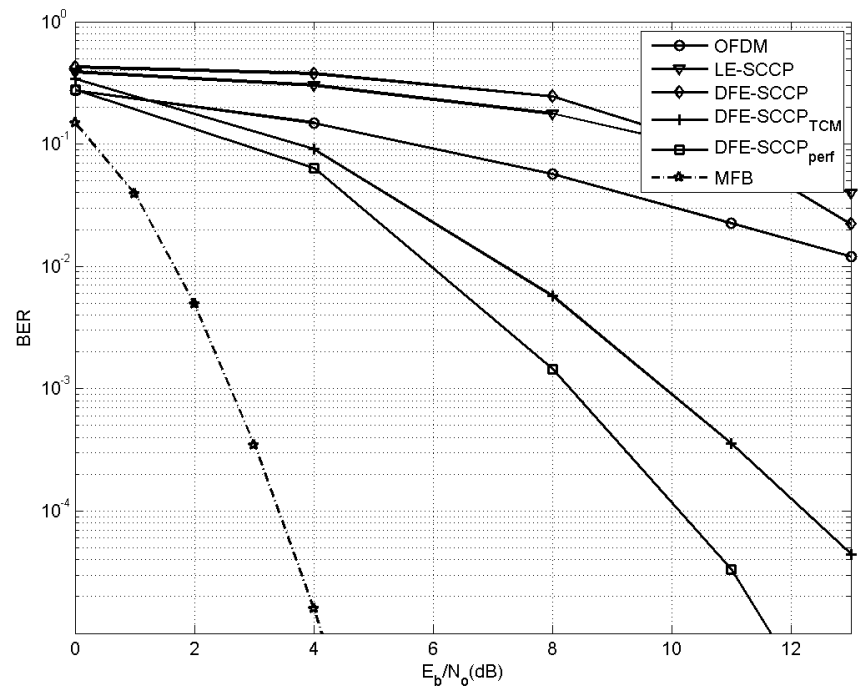

Figura 6.12: Comparação de desempenho entre OFDM e SCCP equalizado com DFE, para o canal Proakis C. Entrelaçador regular com $m=16$ linhas.

portante por fornecer o indicativo de como os sistemas se comportam numa situação de pior caso. Entretanto, como esses canais de difícil equalização ocorrem com uma probabilidade baixa, é também importante analisar como os sistemas se comportam na média de realizações de canal.

Nesta seção, com o objetivo de analisar o desempenho médio dos sistemas, serão considerados canais dispersivos com desvanecimento Rayleigh por blocos. Assumiremos que esses canais têm função de transferência da seguinte forma:

$$
H(z)=h_{0}+h_{1} z^{-1}+h_{2} z^{-2}
$$

em que os coeficientes $h_{k} \sim \mathcal{C N}(0,1 / 3)$.

Primeiramente, será avaliado o impacto da escolha dos diferentes tipos de entrelaçamento nos sistemas. Após a discussão do entrelaçamento, serão estabelecidas curvas de comparação de desempenho quando utilizadas modulação QPSK e 16-QAM.

No decorrer dessa seção utilizaremos o código convolucional de polinômio gerador [133 171$]_{\text {octal }}$ de taxa $R=1 / 2$, bem como o código de taxa $R=3 / 4$ obtido a partir de seu puncionamento [64]. Em todas as simulações realizadas foram considerados blocos com $N=512$ subportadoras. 


\subsubsection{Impacto da escolha do entrelaçador}

A seguir, será analisado como os sistemas se comportam para as diferentes classes de entrelaçadores.

\section{Entrelaçador Regular}

Assim como feito no caso de canais estáticos, a BER de cada um dos sistemas é obtida para entrelaçadores regulares com diferentes configurações. Os resultados, obtidos a partir de vinte mil realizações de canal, estão mostrados na Fig. 6.13.

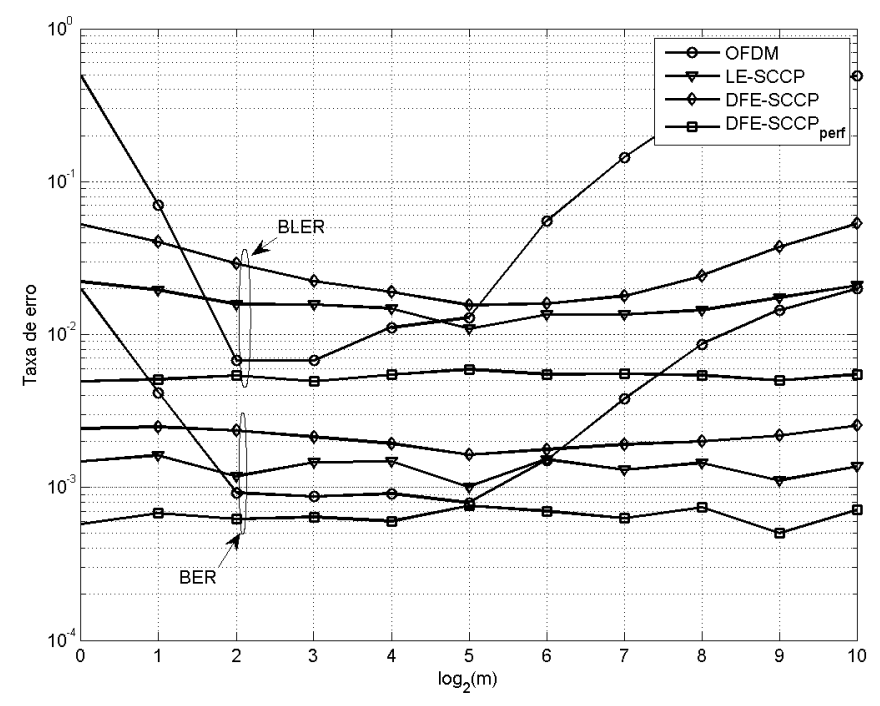

Figura 6.13: Comparação de desempenho - Efeito da configuração do entrelaçador regular no canal com função de transferência dada pela eq. (6.12), modulação QPSK e taxa $R=1 / 2$, $E_{b} / N_{o}=12 \mathrm{~dB}$.

Note que no caso SCCP, a escolha do entrelaçador não traz grandes impactos no desempenho do sistema. No caso OFDM, por outro lado, o projeto do entrelaçador é importante. A BER do pior caso é cerca de dez vezes maior do que aquela atingida quando o entrelaçador apropriado é usado.

\section{Entrelaçador Modular}

Voltando as atenções ao entrelaçador modular, foi determinado qual o parâmetro $N_{1}$ leva ao melhor desempenho dos sistemas. Para os sistemas SCCP, percebeu-se que eles eram insensíveis quanto à escolha do parâmetro $N_{1}$. Os resultados para o sistema OFDM, também gerados a partir de vinte mil realizações de canal, estão mostrados na Fig. 6.14. 


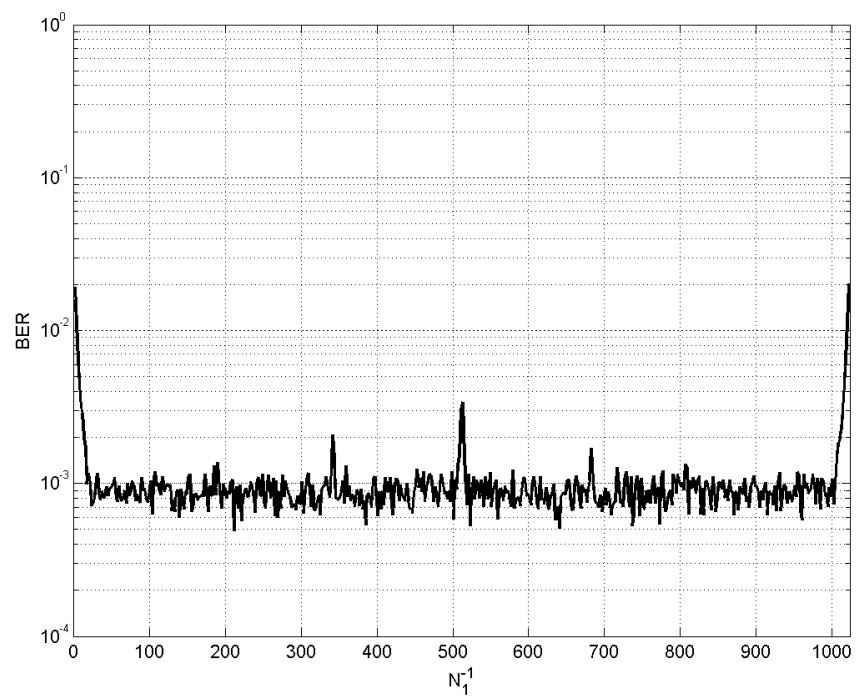

Figura 6.14: Efeito do entrelaçador modular para o sistema OFDM no canal com função de transferência dada pela eq. (6.12), modulação QPSK e taxa $R=1 / 2, E_{b} / N_{o}=12 \mathrm{~dB}$.

Analisando a Fig. 6.14 é observado que a maioria das possíveis escolhas de $N_{1}$ levam ao mesmo desempenho do sistema OFDM. Entretanto, há alguns valores do parâmetro $N_{1}$ para os quais a BER é significativamente maior do que a média.

Primeiramente, se $N_{1}^{-1}$ for próximo de 1, o desempenho do sistema é degradado. A partir da discussão apresentada na seção 6.1 .2 , concluímos que $N_{1}^{-1}$ pequeno indica que a distância imposta pelo entrelaçamento entre dois bits consecutivos na sequência original é pequena. Sendo assim, o entrelaçamento não é eficaz. A circularidade do mapeamento permite que a mesma justificativa seja dada para a queda de desempenho para parâmetros de entrelaçamento $N_{1}^{-1}$ próximos de $N_{b}=1024$.

O fato do parâmetro $N_{1}^{-1}=511$ levar a uma degradação de desempenho quando comparado a outras possíveis escolhas de parâmetros não se enquadra na justificativa dada acima. Entretanto, notando que $N_{1}^{-1}=511 \Leftrightarrow N_{1}=511$, a sequência gerada pelo mapeamento é aquela mostrada na Fig. 6.15. Observa-se, em cada três bits consecutivos da sequência entrelaçada, há dois bit que originalmente eram separados apenas por um terceiro bit. Claramente, essa não é a forma mais eficiente de se entrelaçar.

\section{Entrelaçamento S-aleatório}

Os resultados gerados com diferentes configurações de entrelaçador aleatório estão mostrados na Fig. 6.16. Nela observa-se que os sistemas são praticamente invariantes à escolha do parâmetro $S$. Isto se deve ao fato de que, apenas alguns casos bem especiais de entre- 


\begin{tabular}{c|c|c|c|c|c|c|c|c|c|c|c|c|c|c|}
\hline$X_{0}$ & $X_{1}$ & $X_{2}$ & $X_{3}$ & $X_{4}$ & $X_{5}$ & $X_{6}$ & $X_{7}$ & $\ldots$ & $X_{1118}$ & $X_{1119}$ & $X_{1020}$ & $X_{1021}$ & $X_{1022}$ & $X_{1023}$ \\
Entrelaçamento & \\
\hline$X_{0}$ & $X_{511}$ & $X_{1022}$ & $X_{509}$ & $X_{1020}$ & $X_{507}$ & $X_{1118}$ & $X_{505}$ & $\ldots$ & $X_{6}$ & $X_{517}$ & $X_{4}$ & $X_{515}$ & $X_{2}$ & $X_{513}$ \\
\hline
\end{tabular}

Figura 6.15: Sequência de $N_{b}=1024$ bits entrelaçada com entrelaçador modular com parâmetro $N_{1}^{-1}=511$.

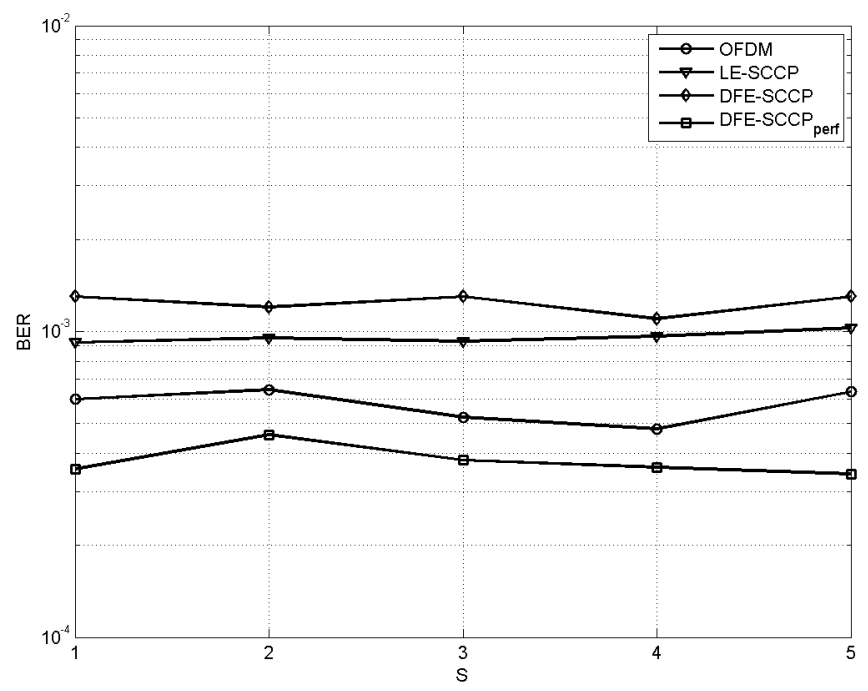

Figura 6.16: Efeito do entrelaçador S-aleatório no canal com função de transferência dada pela eq. (6.12), modulação QPSK e taxa $R=1 / 2, E_{b} / N_{o}=12 \mathrm{~dB}$.

laçamento levam a desempenhos diferentes. A grande maioria dos entrelaçadores geram a mesma BER. Como a probabilidade de gerar um entrelaçador aleatório com essas características especiais é muito pequena, o desempenho do entrelaçador aleatório deve coincidir com o desempenho médio.

Comparando os desempenhos obtidos com as melhores configurações das diferentes classes de entrelaçamento, observa-se que, desde que corretamente dimensionados, todos os entrelaçadores levam ao mesmo desempenho. Como os sistemas são praticamente invariantes ao tipo de entrelaçador, optou-se pela utilização do entrelaçador regular. Analisando a Fig. 6.13, conclui-se que o entrelaçador regular com $m=4$ linhas constitui uma boa opção de entrelaçamento para todos os sistemas. Com essa configuração de entrelaçamento, a comparação 
de desempenho entre os sistemas entrelaçados é mostrada na Fig. 6.17.

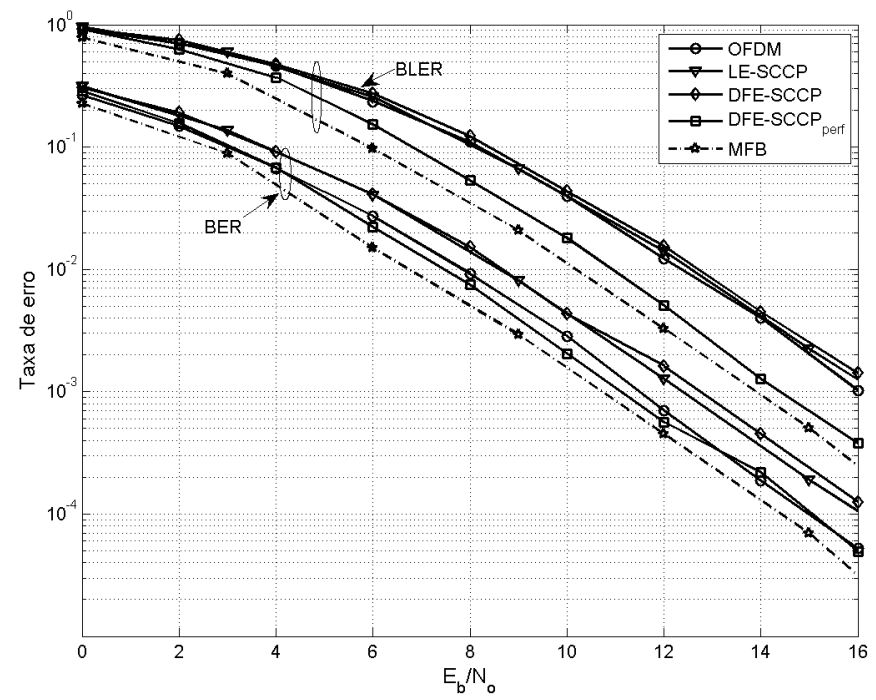

Figura 6.17: Comparação de desempenho - Canal com função de transferência dada pela eq. (6.12), modulação QPSK, taxa $R=1 / 2$, entrelaçador regular com $m=4$ linhas e $n=256$ colunas.

Na Fig. 6.17 nota-se que os sistemas OFDM e LE-SCCP apresentam o mesmo desempenho em termos de BLER, mas o OFDM apresenta um desempenho superior em termos de BER. O que significa que os erros tendem a ocorrer de forma mais espaçada no sistema OFDM do que no sistemas LE-SCCP. Em relação ao DFE, o fenômeno de propagação de erros inerente ao equalizador faz com que o seu desempenho seja ainda pior que o do filtro linear. Já o DFE-SCCP perfeito, neste tipo de canal, tem desempenho comparável com o do OFDM. Tanto o sistema DFE-SCCP perfeito quanto o OFDM, apresentam desempenho próximo ao limitante fornecido pelo MFB.

\subsubsection{Análise com modulação 16-QAM}

Primeiramente, será analisado como o entrelaçador afeta os sistemas quando se utiliza modulação 16-QAM. Para esse tipo de modulação, o decodificador será alimentado com a razão de máxima verossimilhança dos bits enviados obtida a partir do sinal recebido.

Nesta subseção iremos nos ater aos entrelaçadores regulares, pois esse é o tipo de entrelaçamento mais clássico e corresponde a uma classe de entrelaçadores que retrata bem a sensibilidade dos sistemas em relação ao tipo de entrelaçamento utilizado. A Fig. 6.18 mostra como a BER varia em função do parâmetro de entrelaçamento utilizado. Os resultados mostrados nessa figura mostram mais uma vez a sensibilidade do OFDM em relação 


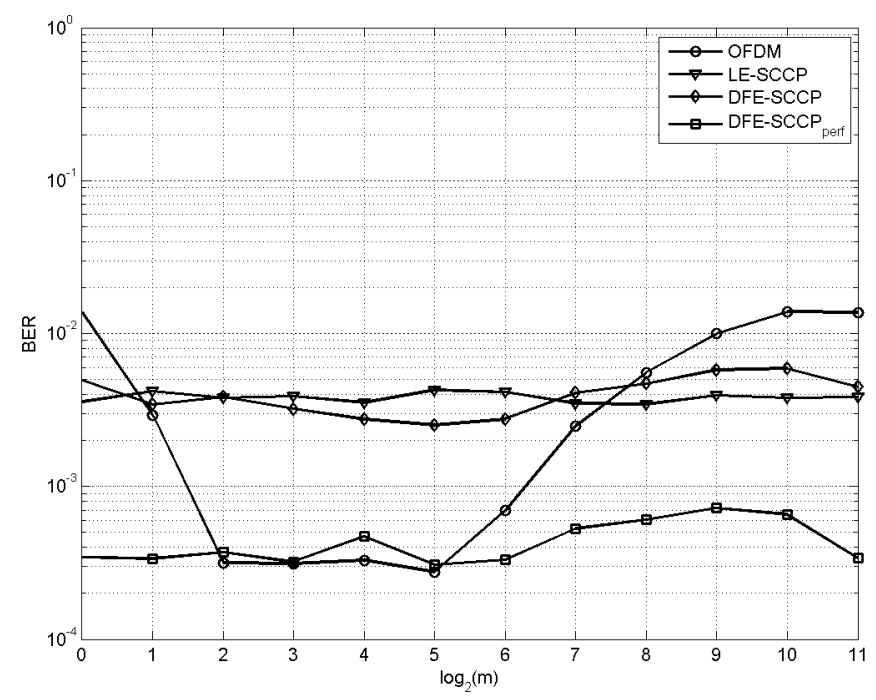

Figura 6.18: Comparação de desempenho - Efeito da configuração do entrelaçador regular no canal com função de transferência dada pela eq. (6.12), modulação 16-QAM, taxa $R=1 / 2$ e $E_{b} / N_{o}=16 \mathrm{~dB}$.

à configuração de entrelaçamento. Um fato que deve ser observado é que, para modulação 16-QAM, o sistema DFE-SCCP é um pouco menos robusto em relação ao parâmetro de entrelaçamento do que quando se usa modulação QPSK.

A próxima etapa da comparação consiste em, dado que é conhecida a configuração de entrelaçamento mais favorável para os sistemas, estabelecer comparações variando a relação $E_{b} / N_{o}$.

$\mathrm{Na}$ análise anterior, foi visto que os sistemas OFDM e LE-SCCP apresentam o mesmo desempenho em termos de BLER para a configuração de canal adotada. Dessa forma, se os resultados apresentados no capítulo 4 também forem válidos em sistemas codificados, devemos esperar que o desempenho do sistema OFDM seja melhor do que o desempenho do LE-SCCP no caso de modulação 16-QAM. De fato, a Fig 6.19 mostra uma considerável degradação do filtro linear em relação ao OFDM. Um outro ponto que merece destaque é que o OFDM passa a apresentar desempenho comparável ao DFE-SCCP perfeito também em termos de BLER.

Mais uma vez, cabe ressaltar que se não for empregado nenhum mecanismo de minimização de propagação de erros no DFE, o DFE, nas condições simuladas, tende a apresentar um desempenho inferior ao do filtro linear.

É ainda interessante confrontar os resultados obtidos com simulação Monte Carlo com aqueles gerados a partir da análise teórica por cutoff rate descrita no capítulo 5. Para o 


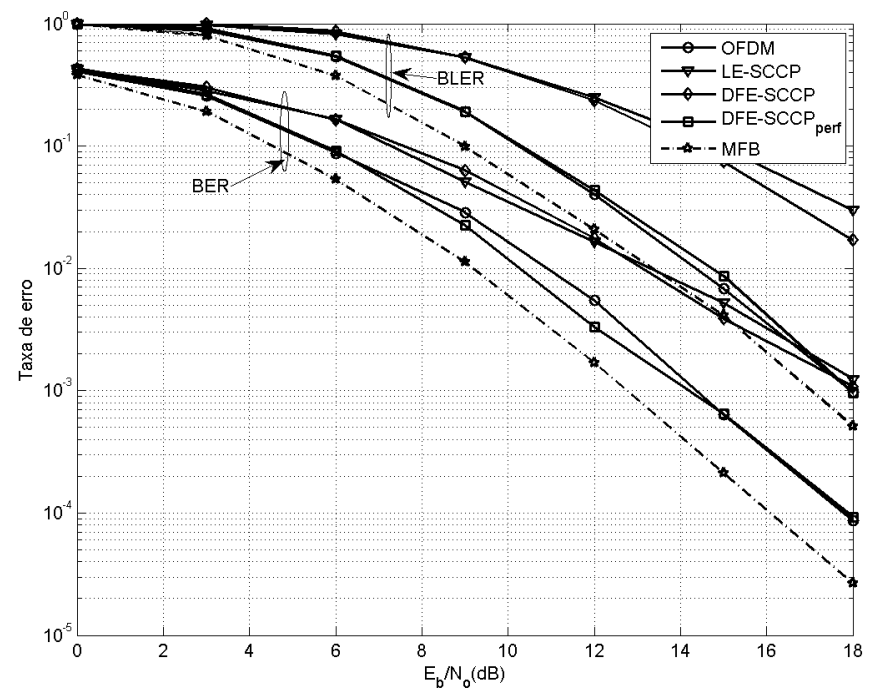

Figura 6.19: Comparação de desempenho - Entrelaçado regular com $m=4$ linhas, canal com função de transferência dada pela eq. (6.12), modulação 16-QAM e taxa $R=1 / 2$.

caso DFE-SCCP, a curva de probabilidade de outage por cutoff rate se aproxima da curva de BLER. Já em relação ao OFDM, a probabilidade de outage por cutoff rate é superior à BLER. Por tal motivo, é dito que nessa situação a cutoff rate é um parâmetro pessimista para o OFDM, i.e., há situações de canal em que há outage por cutoff rate, mas não há erro de bloco. Apesar da aparente contradição, é válido lembrar que a cutoff rate é um limitante inferior da capacidade de Shannon.

\subsubsection{Taxa de codificação}

Nesse contexto, iremos analisar como os sistemas se comportam quando é utilizado um código com maior rendimento. Os resultados obtidos nessa seção foram gerados a partir do código de taxa $\mathrm{R}=3 / 4$ obtido através do puncionamento do código convolucional [133 171$]_{\text {octal }}$ com taxa $\mathrm{R}=1 / 2$.

Através de simulação Monte Carlo, determinou-se, para cada um dos sistemas, qual configuração de entrelaçamento regular leva ao melhor desempenho. A dependência do desempenho do sistema com o entrelaçador é semelhante à observada para o caso com taxa $R=1 / 2$, i.e., enquanto o sistema OFDM é sensível em relação à escolha do padrão de entrelaçamento, os sistemas SCCP são praticamente invariantes em relação à tal escolha. As simulações mostraram que a melhor condição de entrelaçamento regular para o OFDM corresponde ao entrelaçador com $m=32$ linhas. Como o desempenho dos sistemas SCCP 
não é associado ao entrelaçamento, no caso estudado, será utilizado o mesmo padrão de entrelaçamento empregado nos sistemas OFDM.

A princípio, podemos inferir que os resultados nesse contexto deverão se encontrar em uma situação intermediária entre os casos sem codificação e com código de taxa $R=1 / 2$.

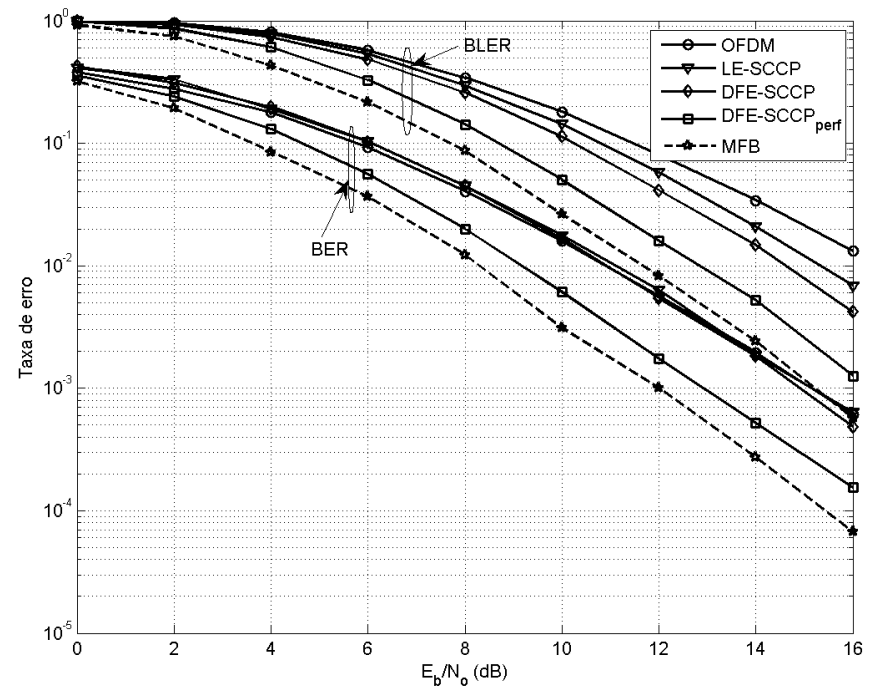

Figura 6.20: Comparação de desempenho - Entrelaçador regular com $m=32$ linhas, canal com função de transferência dada pela eq. (6.12), modulação QPSK e taxa $R=3 / 4$.

Os resultados estão mostrados na Fig. 6.20. Note que neste caso, o desempenho do OFDM, em termos de BER, é equivalente ao desempenho do DFE-SCCP, considerando a propagação de erros, e ao LE-SCCP. Nesse ponto é interessante retomar os resultados obtidos com taxa de codificação $R=1 / 2$. Lembre-se que nessa situação o desempenho do OFDM era equivalente ao do DFE-SCCP perfeito.

Isso corrobora a tese que a dependência do OFDM em relação à taxa de codificação de canal é mais pronunciada do que a do SCCP, como é possível inferir a partir da análise de cutoff rate.

Ainda em relação à cutoff rate, percebe-se que a probabilidade de outage apresentada no capítulo 5 é bem coerente com as curvas de BLER mostradas nessa seção. Nesse caso, outage por cutoff rate implica em erro de bloco.

\section{Sumário}

Neste capítulo foi dada ênfase à configuração do entrelaçador de bits aplicado no processo de codificação e avaliada a sensibilidade de cada um dos sistemas em relação à configura- 
ção de entrelaçamento utilizada. A principal conclusão derivada dessa análise foi a grande suscetibilidade do sistema OFDM à escolha do entrelaçador, em especial no caso de canais estáticos fortemente seletivos em frequência. Observou-se também a robustez apresentada pelo sistema SCCP diante desse mesmo parâmetro.

Um outro aspecto importante abordado nesse capítulo foi a verificação de que os resultados gerados em capítulos anteriores, nos quais foram comparados os sistemas sem aplicação de codificação de canal, capítulo 4, e com códigos aleatórios, capítulo 5, são refletidos também no caso em que são considerados códigos corretores de erros. A única ressalva é feita para o sistema DFE-SCCP que também apresenta degradação em relação ao aumento da cardinalidade da modulação, fato que não pôde ser observado na análise por cutoff rate, mas que foi percebida na análise sem código do capítulo 4.

Finalmente, foi também observado que as diferenças existentes entre a análise onde é considerada transmissão em canal estático e a análise em que é considerada transmissão em canal seletivo em frequência com desvanecimento Rayleigh por blocos. Foi mostrado que há configurações de canais estáticos que não permitem nenhuma configuração de entrelaçamento que leve o sistema OFDM a apresentar resultados compatíveis com o DFE-SCCP. 


\section{Capítulo 7}

\section{Comparação através da SNR efetiva}

No capítulo 4 foram deduzidas expressões para a taxa de erro de bit dos sistemas OFDM e SCCP na ausência de codificação de canal. Em seguida, foram exploradas características de convexidade das funções de probabilidade de erro e, dessa forma, foi possível prover uma análise comparativa para os sistemas estudados.

Nesta capítulo, tem-se como objetivo desenvolver uma análise similar para os sistemas codificados.

Nos sistemas SCCP, considerando uma aproximação gaussiana para a ISI, a probabilidade de erro de bit pode ser obtida mapeando a SNR na saída do equalizador na função da probabilidade de erro de bit para canais AWGN. Entretanto, no caso OFDM, não há uma função que defina tal SNR. Como cada símbolo é sujeito a diferentes ganhos, a princípio, cada um teria uma SNR diferente. No capítulo 4, foi dito que a cada subportadora estaria associada uma diferente BER e, como as subportadoras eram independentes, a BER do sistema seria determinada simplesmente pelo valor médio da BER nas subportadoras individuais.

A partir do momento em que a codificação de canal é empregada, a redundância imposta pelo código torna as subportadoras OFDM dependentes. Nessa situação, a BER do sistema OFDM não pode ser obtida meramente através da média aritmética das BER em cada subportadora.

A dificuldade em se estimar a probabilidade de erro de bit em sistemas OFDM codificados motiva a busca por figuras de mérito de tais sistemas. Para tanto, é preciso obter uma figura de mérito similar a SNR dos sistemas SCCP, no sentido de que a probabilidade de erro de bit seja obtida mapeando tal figura de mérito na função da probabilidade de erro associada ao código. Um possível modo de tratar esse problema é definir como figura de mérito a SNR efetiva $\left(\mathrm{SNR}_{e f f}\right)[65]$. 
Ao longo deste capítulo, será mostrado que tal figura de mérito, de fato, resulta numa métrica consistente com o desempenho do sistema OFDM nas situações detalhadas no decorrer do capítulo. Posteriormente, será estabelecida uma comparação entre os sistemas estudados, assumindo a hipótese de validade do parâmetro de $\mathrm{SNR}_{e f f}$.

\subsection{Definição da SNR efetiva}

Há várias possíveis formas de se definir a $\mathrm{SNR}_{e f f}$. Como mostrado em [66], de um modo geral, as alternativas para definição de $\mathrm{SNR}_{\text {eff }}$ podem ser expressas como:

$$
\mathrm{SNR}_{e f f}=\lambda_{1} I^{-1}\left(\frac{1}{N} \sum_{k=0}^{N-1} I\left(\frac{\mathrm{SNR}_{k}}{\lambda_{2}}\right)\right)
$$

em que $I(\cdot)$ é a função que caracteriza a $\mathrm{SNR}_{e f f}, \mathrm{SNR}_{k}$ é a $\mathrm{SNR}$ na $k$-ésima subportadora e os parâmetros $\alpha_{1}, \alpha_{2}$ são constantes que devem ser ajustas em função, principalmente, das condições de modulação e codificação.

Diferentes escolhas da função $I(\cdot)$, levam a distintas definições para a $\mathrm{SNR}_{\text {eff }}$ [66]. Se $I(\cdot)$ for adotada como $I(x)=\log (1+x)$, tem-se a abordagem via capacidade. Uma segunda possibilidade seria admitir $I(\cdot)$ como uma função de informação mútua, assim como adotado em [67].

A definição mais adotada é a aproximação exponencial, assim como feito em [65] e [68]. Como indicado em [68], tal abordagem é fundamentada a partir da aplicação da cota de Chernoff na função de BER. A $\mathrm{SNR}_{\text {eff }}$ gerada a partir de tal aproximação é definida impondo $I(x)=\exp (-x)$ e $\lambda_{1}=\lambda_{2}=\lambda$ :

$$
\mathrm{SNR}_{e f f}=-\lambda \log \left(\frac{1}{N} \sum_{k=0}^{N-1} \exp \left(-\gamma \frac{\left|H_{k}\right|^{2}}{\lambda}\right)\right)
$$

Essa será a definição adotada ao longo desse capítulo, uma vez que é o padrão mais adotado nas comparações existentes na literatura.

\subsection{BER para sistemas codificados}

A análise dos sistemas SCCP quando a transmissão se dá com o emprego de códigos corretores de erros é similar à análise da transmissão sem codificação. 
Denotando por $Q_{c}(\cdot)$ a função da probabilidade de erro do código em canal AWGN, a BER dos sistemas SCCP, desde que a aproximação gaussiana para a ISI seja válida, é obtida através do mapeamento da SNR na saída do equalizador na função $Q_{c}(\cdot)$.

Se a $\mathrm{SNR}_{\text {eff }}$ for uma aproximação válida para o sistema OFDM, a BER dos sistemas OFDM seria calculada de forma similar à BER dos sistemas SCCP: através do mapeamento da $\mathrm{SNR}_{e f f}$ na função $Q_{c}(\cdot)$.

Em [64] é apresentado um limitante para a probabilidade de erro do código, obtido a partir da distância livre e da distribuição de pesos do código. Para o código utilizado, e modulação de símbolos QPSK, esse limitante é dado por ${ }^{1}$ :

$$
f\left(\frac{E_{b}}{N_{o}}\right)=Q\left(\sqrt{10 \frac{E_{b}}{N_{o}}}\right)\left(36+211 \exp \left(-\frac{E_{b}}{N_{o}}\right)+1404 \exp \left(-2 \frac{E_{b}}{N_{o}}\right)\right)
$$

Esse é um limitante superior e se aproxima da real função da probabilidade de erro para altos valores de SNR. Para baixos valores de SNR, essa aproximação não é mais válida e se faz necessária uma outra solução, relatada a seguir.

Em regime de baixa SNR, é possível estimar a probabilidade de erro através de simulação Monte Carlo. Interpolando os pontos de BER obtidos, é gerada uma função contínua que aproxima a probabilidade de erro de bit para valores de SNR próximos das simulados. Utilizando uma função interpoladora gaussiana, obtivemos a seguinte aproximação:

$$
\begin{aligned}
g\left(\frac{E_{b}}{N_{o}}\right)=a_{1} \exp \left(-\left(\frac{\frac{E_{b}}{N_{o}}-b_{1}}{c_{1}}\right)^{2}\right)+a_{2} \exp \left(-\left(\frac{\frac{E_{b}}{N_{o}}-b_{2}}{c_{2}}\right)^{2}\right) \\
\frac{}{a_{1}=0,5446} a_{2}=0,1945 \\
\hline b_{1}=-0,1612 b_{2}=0,6232 \\
\hline c_{1}=0,8126 \quad c_{2}=0,3884 \\
\hline
\end{aligned}
$$

A Fig. 7.1 mostra que as funções $g\left(E_{b} / N_{o}\right)$ e $f\left(E_{b} / N_{o}\right)$ aproximam bem a função da probabilidade de erro do código. O valor limiar de $E_{b} / N_{o}$ que indica qual função deve ser aplicada é aproximadamente $\left(E_{b} / N_{o}\right)_{\text {lim }}=1,15 \mathrm{~dB}$.

\footnotetext{
${ }^{1} \mathrm{~A}$ relação $E_{b} / N_{o}$ se relaciona com a SNR da seguinte forma: $\mathrm{SNR}=R \log _{2}(M) \frac{E_{b}}{N_{o}}$
} 
Finalmente, a função $Q_{c}\left(E_{b} / N_{o}\right)$ é dada por:

$$
Q_{c}\left(E_{b} / N_{o}\right)=\left\{\begin{array}{l}
a_{1} \exp \left(-\left(\frac{E_{b}}{\frac{N_{o}}{c_{1}}-b_{1}}\right)^{2}\right)+a_{2} \exp \left(-\left(\frac{\frac{E_{b}}{N_{o}}-b_{2}}{c_{2}}\right)^{2}\right), \quad E_{b} / N_{o}<1,15 \mathrm{~dB} \\
Q\left(\sqrt{10 \frac{E_{b}}{N_{o}}}\right)\left(36+211 \exp \left(-\frac{E_{b}}{N_{o}}\right)+1404 \exp \left(-2 \frac{E_{b}}{N_{o}}\right)\right), \quad E_{b} / N_{o} \geq 1,15 \mathrm{~dB}
\end{array}\right.
$$

Em canais seletivos em frequência, a probabilidade de erro de bit será dada por $Q_{c}$ (SNR). Portanto, dada a SNR na saída do equalizador SCCP, podemos estimar a probabilidade de erro de bit através do mapeamento dessa SNR na função $Q_{c}$. Já no caso OFDM, a BER será dada pelo mapeamento da $\mathrm{SNR}_{e f f}$ na função $Q_{c}$.

Para verificar a validade da aproximação para BER do sistema OFDM obtida a partir da $\mathrm{SNR}_{e f f}$, mais uma vez será considerarada transmissão em canal seletivo em frequência com desvanecimento Rayleigh por blocos. Serão analisados canais com $L=3$ e $L=5$ ramos de diversidade.

A Fig. 7.2 mostra que é possível escolher $\lambda$ tal que o mapeamento da $\mathrm{SNR}_{e f f}$ na função $Q_{c}$ forneça uma boa aproximação para a BER do sistema. Note que esse parâmetro não é sensível para os graus de diversidade simulados.

Dado que a BER no sistema pode ser expressa como o mapeamento da $\mathrm{SNR}_{\text {eff }}$ na função $Q_{c}$, podemos utilizar tal grandeza como métrica na comparação entre os dois sistemas estudados. Para tanto, iremos mais uma vez explorar as relações derivadas da desigualdade de Jensen como feito em capítulos anteriores.

É válido ressaltar que ainda foram simulados cenários de canais estáticos com desvanecimento profundo. Nessas situações, entretanto, a $\mathrm{SNR}_{\text {eff }}$ não gerou aproximações adequadas. Então, é importante destacar que os resultados obtidos nas próximas seções são válidos para canais seletivos em frequência com desvanecimento Rayleigh por blocos e que se faz necessário o uso de outras ferramentas para a análise de canais estáticos fortemente seletivos em frequência.

A princípio, será estabelecida a comparação entre o OFDM e LE-SCCP, posteriormente iremos comparar o sistema OFDM com o DFE-SCCP perfeito. 


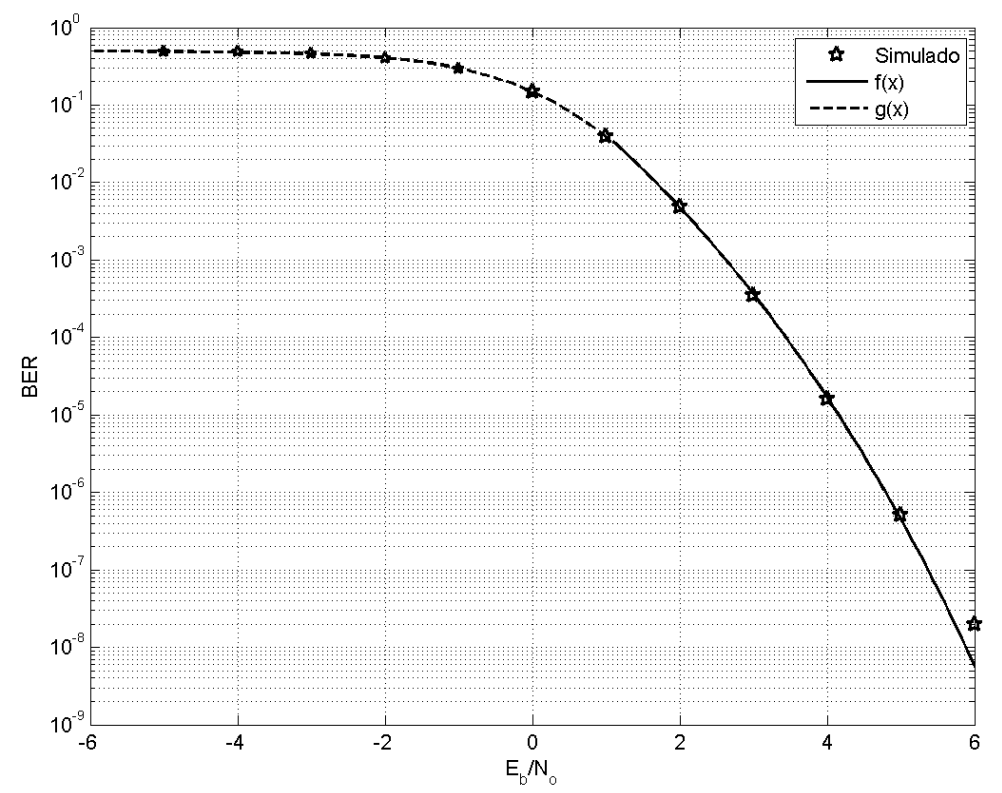

Figura 7.1: Probabilidade de erro de bit em canal AWGN para o código [133 171 $]_{\text {octal }}$.

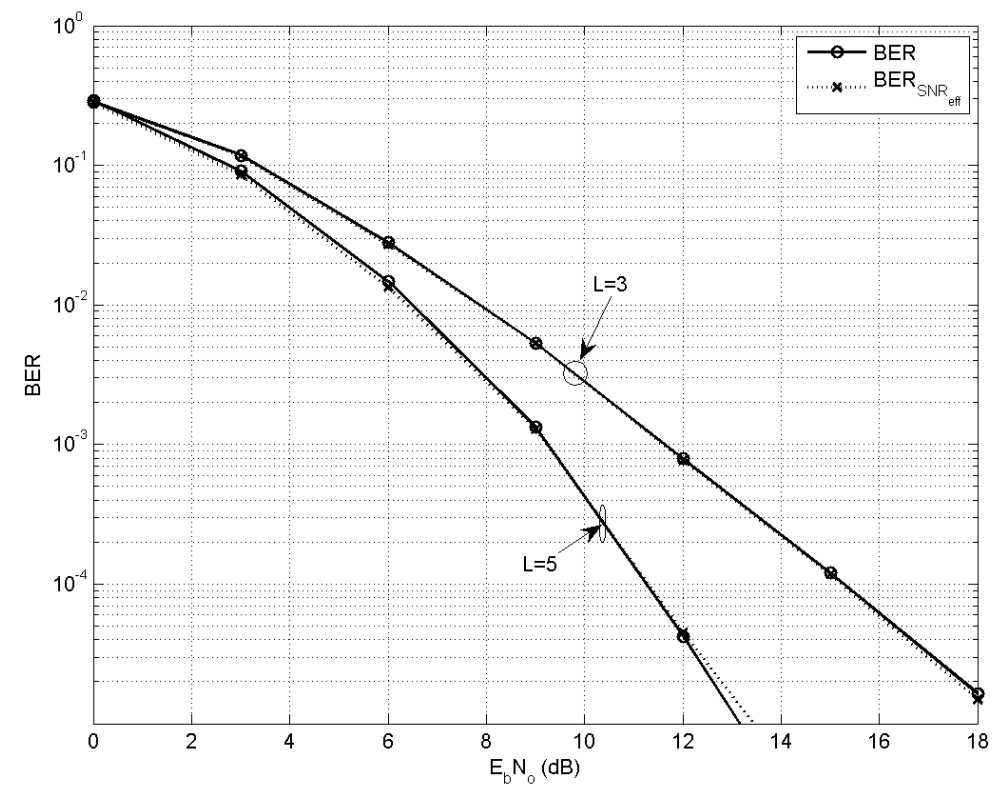

Figura 7.2: Curvas de BER obtidas diretamente através de simulação Monte Carlo e através do mapeamento da $\mathrm{SNR}_{e f f}$ na função $Q_{c}$. Modulação QPSK e canal com desvanecimento Rayleigh por blocos com $L$-coeficientes. Parâmetro $\lambda=1,8$. 


\subsection{Comparação entre os sistemas OFDM e LE-SCCP}

A SNR na saída do equalizador linear é dada por:

$$
\mathrm{SNR}_{L E}=\frac{\frac{1}{N} \sum_{k=0}^{N-1} \frac{\gamma\left|H_{k}^{2}\right|}{\gamma\left|H_{k}\right|^{2}+1}}{\frac{1}{N} \sum_{k=0}^{N-1} \frac{1}{\gamma\left|H_{k}\right|^{2}+1}}
$$

em que $\gamma=\sigma_{X}^{2} / \sigma_{v}^{2}$.

Queremos comparar a SNR dada pela eq. (7.6) com a $\mathrm{SNR}_{e f f}$, dada pela eq. (7.2).

Sabemos que a $\mathrm{SNR}_{\mathrm{LE}}$ pode ser expressa em termos da função $\theta(x)=\frac{1}{x+1}$ :

$$
\mathrm{SNR}_{L E}=\theta^{-1}\left(\frac{1}{N} \sum_{k=0}^{N-1} \theta\left(\gamma\left|H_{k}\right|^{2}\right)\right)
$$

Para proceder com a comparação, é conveniente definir $\varphi(x)=\exp \left(\frac{-x}{\lambda}\right)$. Escrevendo a $\mathrm{SNR}_{\text {eff }}$ em termos da função $\varphi(x)$, pode-se escrever:

$$
\mathrm{SNR}_{e f f}=\varphi^{-1}\left(\frac{1}{N} \sum_{k=0}^{N-1} \varphi\left(\gamma\left|H_{k}\right|^{2}\right)\right)
$$

Aplicando a função $\theta(x)$ nas eqs. (7.8) e (7.7), obtemos:

$$
\begin{aligned}
\theta\left(\mathrm{SNR}_{L E}\right) & =\frac{1}{N} \sum_{k=0}^{N-1} \theta\left(\gamma\left|H_{k}\right|^{2}\right) \\
\theta\left(\mathrm{SNR}_{e f f}\right) & =\theta\left(\varphi^{-1}\left(\frac{1}{N} \sum_{k=0}^{N-1} \varphi\left(\gamma\left|H_{k}\right|^{2}\right)\right)\right)
\end{aligned}
$$

Nesse ponto, vale lembrar que a função $\theta(x)$ é estritamente decrescente. Logo, para determinar se $\mathrm{SNR}_{L E}>\mathrm{SNR}_{e f f}$ basta verficar se $\theta\left(\mathrm{SNR}_{L E}\right)<\theta\left(\mathrm{SNR}_{e f f}\right)$. Para tanto, pode-se definir a função $\mu(x)=\theta\left(\varphi^{-1}(x)\right)$ e escrever as eqs. (7.9) e (7.10) em termos de $\mu(x)$ :

$$
\begin{array}{r}
\theta\left(\mathrm{SNR}_{L E}\right)=\frac{1}{N} \sum_{k=0}^{N-1} \mu\left(\varphi\left(\gamma\left|H_{k}\right|^{2}\right)\right) \\
\theta\left(\mathrm{SNR}_{e f f}\right)=\mu\left(\frac{1}{N} \sum_{k=0}^{N-1} \varphi\left(\gamma\left|H_{k}\right|^{2}\right)\right)
\end{array}
$$


Se a função $\mu(x)$ for convexa, a desigualdade de Jensen garante que $\mu\left(\frac{1}{N} \sum_{k=0}^{N-1} \varphi\left(\gamma\left|H_{k}\right|^{2}\right)\right) \leq \mu\left(\frac{1}{N} \sum_{k=0}^{N-1} \varphi\left(\gamma\left|H_{k}\right|^{2}\right)\right)$. Para analisar a convexidade de $\mu(x)$, é preciso obter sua segunda derivada:

$$
\mu(x)=\frac{1}{1-\lambda \log (x)} \Rightarrow \frac{d^{2}}{d x^{2}} \mu(x)=\frac{\lambda}{x^{2}(1-\lambda \log (x))^{2}}\left(-1+\frac{2}{1-\lambda \log (x)}\right)
$$

De acordo com a eq. (7.11), a SNR do sistema LE-SCCP é determinada a partir da aplicação da função $\mu(x)$ em $x_{k}=\varphi\left(\gamma\left|H_{k}\right|^{2}\right)=\exp \left(-\frac{\gamma\left|H_{k}\right|^{2}}{\lambda}\right)$, que é um argumento menor do que um. Do mesmo modo, de acordo com a eq. (7.12), a SNR do sistema OFDM é obtida a partir da aplicação de $\mu(x)$ no valor médio de $\varphi\left(\gamma\left|H_{k}\right|^{2}\right)$, que também é menor do que a unidade. Assim, é preciso apenas analisar como se comporta $\frac{d^{2}}{d x^{2}} \mu(x)$ no intervalo $x \in\left[\begin{array}{ll}0 & 1\end{array}\right]$.

Se o parâmetro que melhor ajusta a $\mathrm{SNR}_{e f f}$ do OFDM for $\lambda<0,5$, a segunda derivada da função $\mu(x)$ é positiva qualquer que seja a condição de canal. Isso significa que $\mu(x)$ é uma função convexa e $\mathrm{SNR}_{e f f}>\mathrm{SNR}_{L E}$. Entretanto, os resultados mostrados na Fig. 7.2 mostram que, no contexto de canal seletivo em frequência com desvanecimento Rayleigh por blocos, o valor ótimo do parâmetro $\lambda$ é aproximadamente 2. Portanto, essa condição $\lambda<0,5$ não parece ter um significado físico importante para as condição de codificação e modulação analisada.

Se $\lambda>0,5$, a função $\mu(x)$ é convexa para $x \in\left[0, e^{1 / \lambda-2}\right]$ e côncava para $x \in\left[e^{1 / \lambda-2}, 1\right]$. Como a função $\mu(x)$ apresenta intervalos de concavidade e convexidade em seu domínio, a princípio, não podemos concluir a respeito da comparação entre $\mathrm{SNR}_{\text {eff }}$ e $\mathrm{SNR}_{L E}$. Todavia, sabendo que a função $\mu(x)$ é calculada em $x_{k}=\exp \left(-\frac{\gamma\left|H_{k}\right|^{2}}{\lambda}\right)$, pode-se encontrar um limiar de SNR $\gamma_{\text {lim }}$, tal que $\gamma>\gamma_{\text {lim }}$ garante que $\mathrm{SNR}_{\text {eff }}>\mathrm{SNR}_{L E}$. Para que todas as subportadoras estejam na região de convexidade é necessário que:

$$
\exp \left(-\frac{\gamma\left|H_{k}\right|^{2}}{\lambda}\right) \leq e^{1 / \lambda-2}, \forall k
$$

Logo, o valor de SNR $\gamma_{\text {lim }}$ é dado por:

$$
\gamma_{l i m}=\max _{k} \frac{2 \lambda-1}{\left|H_{k}\right|^{2}}
$$




\subsection{Comparação entre os sistemas OFDM e DFE-SCCP}

Nesse seção, serão compararados os sistemas DFE-SCCP e OFDM de modo semelhante ao realizado para os sistemas LE-SCCP e OFDM. Para tanto, reescreve-se a $\mathrm{SNR}_{D F E}$ em termos da função $\phi(x)=\log (x+1)$ :

$$
\mathrm{SNR}_{D F E}=\exp \left\{\frac{1}{N} \sum_{k=0}^{N-1} \log \left(1+\gamma\left|H_{k}\right|^{2}\right)\right\}-1=\phi^{-1}\left(\frac{1}{N} \sum_{k=0}^{N-1} \phi\left(\gamma\left|H_{k}\right|^{2}\right)\right)
$$

Aplicando a função $\phi(x)$ em (7.8) e (7.16), temos:

$$
\begin{aligned}
\phi\left(\mathrm{SNR}_{D F E}\right) & =\frac{1}{N} \sum_{k=0}^{N-1} \phi\left(\gamma\left|H_{k}\right|^{2}\right) \\
\phi\left(\mathrm{SNR}_{e f f}\right) & =\phi\left(\varphi^{-1}\left(\frac{1}{N} \sum_{k=0}^{N-1} \varphi\left(\gamma\left|H_{k}\right|^{2}\right)\right)\right)
\end{aligned}
$$

Definindo $\kappa(x)=\phi\left(\varphi^{-1}(x)\right)$, as eqs. (7.17) e (7.17) podem ser reescritas em função de $\kappa(x)$ :

$$
\begin{aligned}
& \phi\left(\mathrm{SNR}_{D F E}\right)=\frac{1}{N} \sum_{k=0}^{N-1} \kappa\left(\varphi\left(\gamma\left|H_{k}\right|^{2}\right)\right) \\
& \phi\left(\mathrm{SNR}_{e f f}\right)=\kappa\left(\frac{1}{N} \sum_{k=0}^{N-1} \varphi\left(\gamma\left|H_{k}\right|^{2}\right)\right)
\end{aligned}
$$

Diferentemente da função $\theta(x)$, a função $\phi(x)$ é estritamente crescente. Portanto, $\phi\left(\mathrm{SNR}_{e f f}\right)<$ $\phi\left(\mathrm{SNR}_{D F E}\right) \Rightarrow \mathrm{SNR}_{e f f}<\mathrm{SNR}_{D F E}$. Logo, aplicando a Desigualdade de Jensen nas eqs. (7.19) e (7.20), conclui-se que se $\kappa(x)$ for convexa, a $\mathrm{SNR}_{e f f}$ é menor do que a $\mathrm{SNR}_{D F E}$.

A função $\kappa(x)$ é dada por:

$$
\kappa(x)=\log (1-\lambda \log (x))
$$

e sua segunda derivada é dada por:

$$
\frac{d^{2}}{d x^{2}} \kappa(x)=\frac{\lambda}{x^{2}(1-\lambda \log (x))}\left(1-\frac{\lambda}{1-\lambda \log (x)}\right)
$$


Nesse ponto é necessário enfatizar que o intervalo do domínio de interesse da função $\kappa(x)$ corresponde ao intervalo $[0,1]$, já que a função é calculada em $x_{k}=\exp \left(-\frac{\gamma\left|H_{k}\right|^{2}}{\lambda}\right), \in[0,1]$. Desse modo, $\log (x)$ é um termo estritamente negativo no intervalo de interesse. Portanto, pode-se afirmar que:

1. $\frac{\lambda}{x^{2}(1-\lambda \log (x))}>0$

2. $1-\lambda \log (x)>\lambda$

A partir desses dois argumentos, conclui-se que $\frac{d^{2}}{d x^{2}} \kappa(x)>0$ para qualquer que seja o parâmetro $\lambda$, ou seja, a função $\kappa(x)$ é convexa e $\mathrm{SNR}_{\text {eff }} \leq \mathrm{SNR}_{D F E}$.

Finalmente, se for válida a hipótese de que a BER do OFDM possa ser mapeada a partir de sua $\mathrm{SNR}_{e f f}$, o sistema SCCP equalizado com DFE livre de propagação de erros sempre apresenta um desempenho superior ao do OFDM, independente do valor de $\lambda$ adequado para o sistema OFDM.

\section{Sumário}

Neste capítulo foi mostrado como aplicar os conceitos de $\mathrm{SNR}_{e f f}$ para o cálculo da BER do sistema OFDM codificado.

Mostrou-se ainda que, para canais seletivos em frequência com desvanecimento Rayleigh, a aproximação fornecida pela $\mathrm{SNR}_{\text {eff }}$ gera resultados próximos daqueles obtidos com simulação Monte Carlo.

Assumindo a validade da $\mathrm{SNR}_{\text {eff }}$ enquanto figura de mérito para o sistema OFDM, mostrou-se que o desempenho do sistema DFE-SCCP perfeito é sempre superior ao do sistema OFDM, independentemente da modulação utilizada. Esse fato está de acordo com os resultados de simulação mostrados no capítulo 6 , em que em canais seletivos em frequência com desvanecimento Rayleigh, o desempenho do OFDM nunca supera o do sistema DFESCCP perfeito. Nos resultados obtidos para tais canais, apesar do aumento da ordem da modulação fazer com que o desempenho do OFDM se aproxime do DFE-SCCP perfeito, não foram verificadas situações em que o OFDM supera DFE-SCCP perfeito.

No capítulo 6, a única situação em que o OFDM supera o DFE-SCCP perfeito se refere à transmissão em canal estático fortemente seletivo em frequência, i.e., uma situação na qual a $\mathrm{SNR}_{e f f}$ não é uma boa aproximação. 



\section{Capítulo 8}

\section{Sensibilidade a erros de estimação de}

\section{canal}

Ao longo da dissertação, foi considerado que a estimação de canal na recepção era livre de erros. Resta, então, analisar as consequências quando tal fato não é verificado. A análise será feita assumindo que o canal estimado na recepção corresponde ao canal livre de erros perturbado por ruído gaussiano complexo. Assim, adotando um estimador ML [69], a $k$-ésima componente em frequência do canal é dada por:

$$
H_{k, e s t}=H_{k}+\Delta_{k}
$$

em que $\Delta_{k} \sim \operatorname{e} \mathcal{N}\left(0, \sigma_{\Delta}^{2}\right)$ e $\mathrm{E}\left\{\Delta_{k} H_{k}\right\}=0$.

Partindo dessa hipótese, serão verificadas as curvas de taxa de erro de bit para os dois sistemas em estudo.

\subsection{Efeito do erro de estimação de canal no sistema OFDM}

De acordo com o desenvolvimento realizado nos capítulos anteriores, o sinal recebido na $k$-ésima subportadora OFDM é dado por:

$$
Y_{k}=H_{k} X_{k}+\Upsilon_{k}
$$


e o sinal na saída do equalizador do tipo filtro-casado pode ser escrito como:

$$
Z_{k}=H_{k, e s t}^{*} Y_{k}
$$

Assumindo que $H_{k, \text { est }}=H_{k}+\Delta_{k}$, tem-se:

$$
\begin{aligned}
Z_{k} & =\left(H_{k}+\Delta_{k}\right)^{*} H_{k} X_{k}+\left(H_{k}+\Delta_{k}\right)^{*} \Upsilon_{k} \\
& =\left|H_{k}\right|^{2} X_{k}+\underbrace{H_{k} \Delta_{k}^{*} X_{k}+H_{k}^{*} \Upsilon_{k}+\Delta_{k} \Upsilon_{k}}_{\text {interf }}
\end{aligned}
$$

A variância do termo interferente interf na eq. (8.5) é dada por:

$$
\sigma_{\text {interf }}^{2}=\left|H_{k}\right|^{2} \sigma_{\Delta}^{2} \sigma_{X}^{2}+\left|H_{k}\right|^{2} \sigma_{\Upsilon}^{2}+\sigma_{\Upsilon}^{2} \sigma_{\Delta}^{2}
$$

A SNR na $k$-ésima subportadora será denotada por $\mathrm{SNR}_{\Delta, k}$, apenas para diferenciar da $\mathrm{SNR}_{k}$ obtida no caso de estimação perfeita de canal.

Nesse ponto é válido ressaltar que a distribuição do fator interferente $\Delta_{k} \Upsilon_{k}$ não é gaussiana, já que a função densidade de probabilidade de uma variável aleatória gerada a partir do produto de gaussianas é dada pela função de Bessel modificada de segunda espécie $K_{0}(x)$. Por simplicidade, assumindo que esse fator também tem distribuição gaussiana, pode-se calcular a BER do sistema através da sua $\mathrm{SNR}_{\Delta, k}$ dada por:

$$
\mathrm{SNR}_{\Delta, k}=\frac{\left|H_{k}\right|^{4} \sigma_{X}^{2}}{\left|H_{k}\right|^{2} \sigma_{\Delta}^{2} \sigma_{X}^{2}+\left|H_{k}\right|^{2} \sigma_{\Upsilon}^{2}+\sigma_{\Upsilon}^{2} \sigma_{\Delta}^{2}}
$$

ou ainda:

$$
\mathrm{SNR}_{\Delta, k}=\frac{\left|H_{k}\right|^{2} \gamma}{1+\sigma_{\Delta}^{2}\left(\gamma+\frac{1}{\left|H_{k}\right|^{2}}\right)}
$$

Definindo $\Gamma_{k}$ como:

$$
\Gamma_{k}=\frac{1}{1+\sigma_{\Delta}^{2}\left(\gamma+\frac{1}{\left|H_{k}\right|^{2}}\right)}
$$

e reconhecendo $\left|H_{k}\right|^{2} \gamma$ como a SNR na $k$-ésima subportadora no contexto de perfeita estimação de canal, tem-se que:

$$
\mathrm{SNR}_{\Delta, k}=\Gamma_{k} \mathrm{SNR}_{k}
$$

A eq. (8.10) indica que a SNR na $k$-ésima subportadora pode ser vista como a SNR obtida no contexto de estimação perfeita de canal atenuada por um fator $\Gamma_{k}$. 
Ainda é válido enfatizar que o fator de atenuação $\Gamma_{k}$ é estritamente decrescente em relação à potência de ruído $\sigma_{\Upsilon}^{2}$, o que indica que a diminuição da potência de ruído sempre leva a um aumento da SNR na saída do equalizador. Apesar dessa observação, a princípio, parecer óbvia, será mostrado mais adiante que esse fato não é válido no caso MMSE SCCP.

Um outro ponto importante a ser analisado é o comportamento assintótico da $\mathrm{SNR}_{\Delta, k}$ que ocorre quando $\gamma$ tende para o infinito:

$$
\operatorname{SNR}_{\Delta, k}^{\infty}=\lim _{\gamma \rightarrow \infty} \frac{\left|H_{k}\right|^{2} \gamma}{1+\sigma_{\Delta}^{2}\left(\gamma+\frac{1}{\left|H_{k}\right|^{2}}\right)}=\frac{\left|H_{k}\right|^{2}}{\sigma_{\Delta}^{2}}
$$

A partir da $\mathrm{SNR}_{\Delta, k}$ pode-se calcular a BER do sistema. No caso não codificado, a BER será dada por:

$$
\begin{aligned}
B E R_{O F D M, \Delta} & =\alpha \frac{1}{N} \sum_{k=0}^{N-1} Q\left(\sqrt{\beta \mathrm{SNR}_{\Delta, k}}\right) \\
& =\alpha \frac{1}{N} \sum_{k=0}^{N-1} Q\left(\sqrt{\beta \frac{\left|H_{k}\right|^{2} \gamma}{1+\sigma_{\Delta}^{2}\left(\gamma+\frac{1}{\left|H_{k}\right|^{2}}\right)}}\right)
\end{aligned}
$$

Já no caso assintótico, a BER tende a um patamar de erro dado por:

$$
B E R_{O F D M, \Delta}^{\infty}=\lim _{\gamma \rightarrow \infty} \alpha \frac{1}{N} \sum_{k=0}^{N-1} Q\left(\sqrt{\beta \frac{\left|H_{k}\right|^{2} \gamma}{1+\sigma_{\Delta}^{2}\left(\gamma+\frac{1}{\left|H_{k}\right|^{2}}\right)}}\right)=\alpha \frac{1}{N} \sum_{k=0}^{N-1} Q\left(\sqrt{\beta \frac{\left|H_{k}\right|^{2}}{\sigma_{\Delta}^{2}}}\right)
$$

A fim de validar os resultados teóricos obtidos, considera-se transmissão OFDM sem codificação de canal com modulação QPSK no canal com função transferência dada por:

$$
H(z)=0,0854+0,854 z^{-1}+0,5126 z^{-2}
$$

A fig. 8.1 mostra a BER obtida através de simulação Monte-Carlo e a BER fornecida pela eq. (8.12). Além disso, mostra-se o patamar de erro dado pela eq. (8.14). Observou-se que os resultados teóricos são consistentes e estão de acordo com os resultados gerados através de simulação. A diferença observada no regime de valores moderados de SNRs entre os resultados teóricos e práticos pode ser atribuída à aproximação gaussiana imposta ao termo interferente $\Upsilon_{k} \Delta_{k}$. 


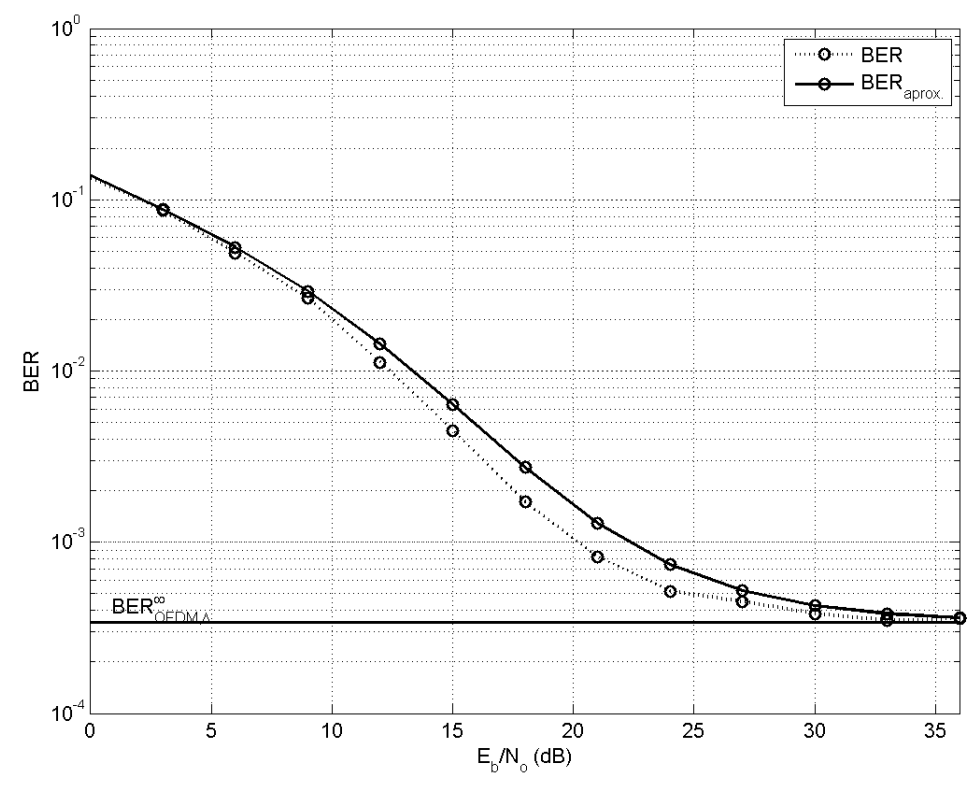

Figura 8.1: BER para o sistema OFDM sem codificação de canal. Modulação QPSK, ruído de estimação de canal com variância $\sigma_{\Delta}^{2}=0,01$ e canal com função de transferência dada por (8.15).

Pode-se ainda analisar o caso com codificação de canal a partir da aproximação fornecida pela $\mathrm{SNR}_{e f f}$. Nesse caso, optaremos por analisar canais seletivos em frequência com desvanecimento Rayleigh por blocos, uma vez que a $\mathrm{SNR}_{e f f}$ se mostra uma aproximação mais coerente nesse contexto do que em canais estáticos. Para tanto, considera-se o canal com função transferência dada pela eq.(6.12).

Os resultados estão expostos na Fig. 8.2 e, assim como no caso de canal estático, indicam uma concordância entre a simulação e o desenvolvimento teórico.

\subsection{Efeito do erro de estimação de canal no sistema SCCP}

Nesta seção serão analisados os efeitos de erros de estimação de canal no sistema SCCP. No capítulo 3, foi mostrado que a estimativa do $k$-ésimo símbolo do bloco SCCP pode ser expressa como:

$$
\hat{X}_{k}=\frac{1}{N} \sum_{n=0}^{N-1}\left[W_{n} H_{n} \sum_{m=0}^{N-1} X_{m} e^{-j \frac{2 \pi}{N} n m}\right] e^{j \frac{2 \pi}{N} k n}+\frac{1}{N} \sum_{n=0}^{N-1} W_{n} \Upsilon_{n} e^{j \frac{2 \pi}{N} k n}
$$

Assumindo critério de equalização MMSE e erros de estimação do canal, o n-ésimo coe- 


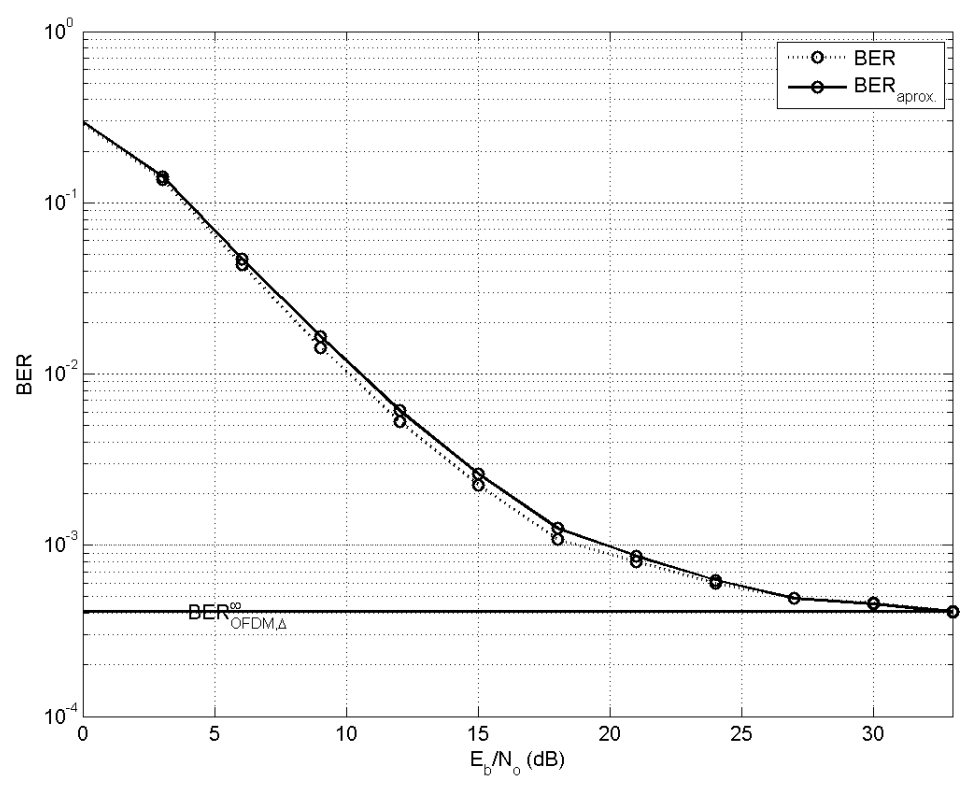

Figura 8.2: BER para o sistema OFDM com codificação de canal (código convolucional [133 171$]_{\text {octal }}$ ) ruído de estimação de canal com variância $\sigma_{\Delta}^{2}=0,05$ e canal seletivo em frequência por blocos com 3 ramos de diversidade.

ficiente de equalização é dado por:

$$
W_{n}=\frac{H_{n}^{*}+\Delta_{n}^{*}}{\left|H_{n}+\Delta_{n}\right|^{2}+\frac{\sigma_{\Upsilon}^{2}}{\sigma_{X}^{2}}}
$$

Substituindo a eq. (8.17) na eq. (8.16) e isolando os termos interferentes, tem-se que:

$$
\begin{aligned}
\hat{X}_{k} & =\underbrace{\frac{1}{N}\left[\sum_{n=0}^{N-1} \frac{\left|H_{n}\right|^{2}}{\left|H_{n}+\Delta_{n}\right|^{2}+\frac{\sigma_{\Upsilon}^{2}}{\sigma_{X}^{2}}}\right]}_{\text {signal }} X_{k} \\
& +\underbrace{\frac{1}{N}\left[\sum_{n=0}^{N-1} \frac{H_{n} \Delta_{n}^{*}}{\left|H_{n}+\Delta_{n}\right|^{2}+\frac{\sigma_{\Upsilon}^{2}}{\sigma_{X}^{2}}}\right] X_{k}+\frac{1}{N} \sum_{\substack{n=0 \\
n \neq k}}^{N-1}\left[\sum_{m=0}^{N-1} \frac{H_{m}^{*}+\Delta_{m}^{*}}{\left|H_{m}+\Delta_{m}\right|^{2}+\frac{\sigma_{\Upsilon}^{2}}{\sigma_{X}^{2}}} H_{m} e^{j \frac{2 \pi}{N}(k-n) m}\right] X_{n}}_{\text {interf }} \\
& +\underbrace{\frac{1}{N} \sum_{n=0}^{N-1} \frac{H_{n}^{*}+\Delta_{n}^{*}}{\left|H_{n}+\Delta_{n}\right|^{2}+\frac{\sigma_{\Upsilon}^{2}}{\sigma_{X}^{2}}} \Upsilon_{n} e^{j \frac{2 \pi}{N} k n}}_{\text {noise }}
\end{aligned}
$$


Observe que, nesse caso, não é possível admitir a aproximação gaussiana. Primeiramente, o ganho do sinal de interesse não é uma constante. Esse ganho é uma variável aleatória cuja densidade de probabilidade nos é desconhecida. Além disso, os termos interferentes também não podem ser aproximados por gaussianas. Ainda, mesmo que se tivesse sido adotado o critério ZF, a expressão do sinal estimado seria um pouco mais simples. Todavia, mesmo nessas condições, ainda não seria possível aplicar a aproximação gaussiana.

No caso do sistema DFE-SCCP, seria ainda mais difícil determinar uma expressão analítica para a BER.

Diante dessas dificuldades, foge do escopo do nosso trabalho determinar uma expressão teórica para a BER do sistema SCCP caso haja erro de estimação de canal. Por tal motivo, os resultados gerados para os sistemas SCCP serão restritos a simulação Monte Carlo.

\subsection{Comparação OFDM vs SCCP}

Mais uma vez, considera-se canais com três coeficientes Rayleigh descorrelacionados, modulação QPSK e código de taxa $R=1 / 2$. A Fig. 8.3 mostra que, para valores baixos de SNR, os sistemas se comportam de forma semelhante ao observado no caso em que a estimação de canal na recepção é perfeita, i.e., o sistema OFDM apresenta desempenho um pouco inferior ao do sistema DFE-SCCP perfeito e os sistemas LE-SCCP e DFE-SCCP apresentam desempenhos comparáveis. Para valores de $E_{b} / N_{o}$ variando entre 10 e $30 \mathrm{~dB}$, a propagação de erros do DFE-SCCP faz com que seu desempenho seja inferior ao do sistema LE-SCCP. Já para valores de $E_{b} / N_{o}$ acima de $30 \mathrm{~dB}$, o sistema DFE-SCCP apresenta uma BER menor do que o do LE-SCCP. Em relação ao OFDM, note que para $E_{b} / N_{o}$ acima de $18 \mathrm{~dB}$, a BER gerada pelo sistema é inferior àquela produzida pelo DFE-SCCP perfeito.

Um outro fato que merece ser destacado é que enquanto a BER do OFDM converge monotonicamente para um patamar de erro, a BER dos sistemas SCCP não é estritamente decrescente com a $E_{b} / N_{o}$. Esse comportamento não monotônico vem do equalizador MMSE e da complexa relação do sinal estimado com o ruído de estimação, como indicado na eq.(8.18).

Como uma segunda comparação, para o mesmo canal com desvanecimento Rayleigh e três coeficientes, considera-se a relação $E_{b} / N_{o}$ fixada em $12 \mathrm{~dB}$ e a BER é estimada através de simulação Monte Carlo. Os resultados estão mostrados na Fig. 8.4 e indicam que a degradação da BER provocada pelo aumento da potência do erro de estimação evolui praticamente da mesma forma para todos os sistemas. Entretanto, nas situações em que a potência de erro de estimação é muito elevada, os sistemas SCCP tendem a ser mais sensíveis ao aumento da potência de erro de estimação do que o sistema OFDM. 


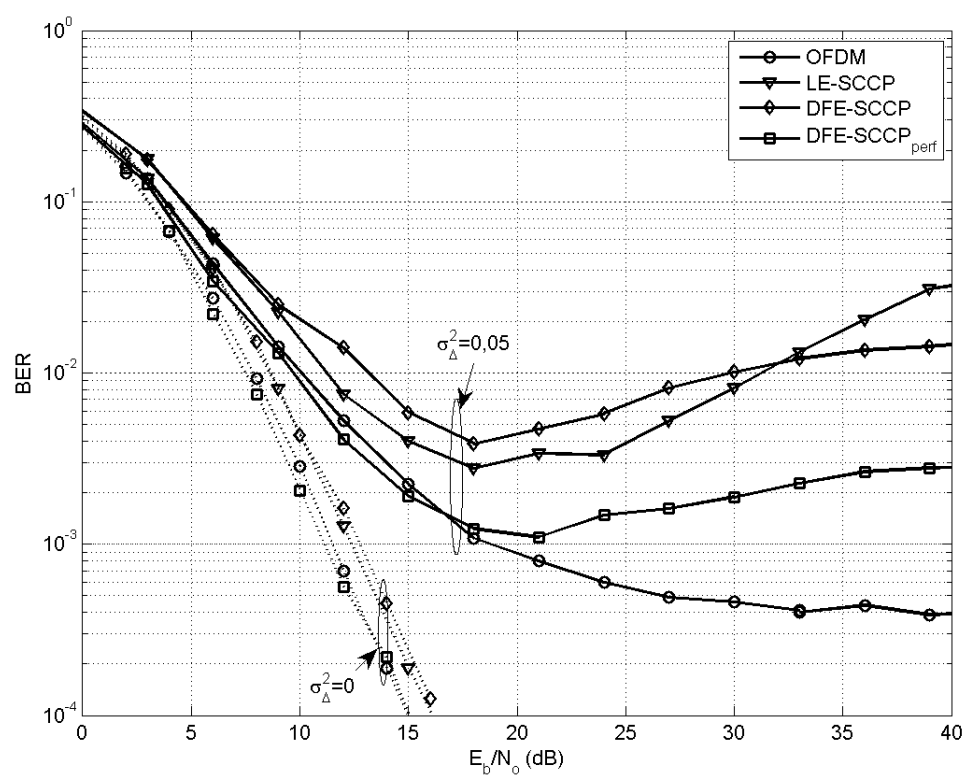

Figura 8.3: Comparação de desempenho. Transmissão QPSK em canal seletivo em frequência com desvanecimento Rayleigh de diversidade $L=3$. Contextos com ruído de estimação de canal de variância $\sigma_{\Delta}^{2}=0,05$ e estimação de canal perfeita.

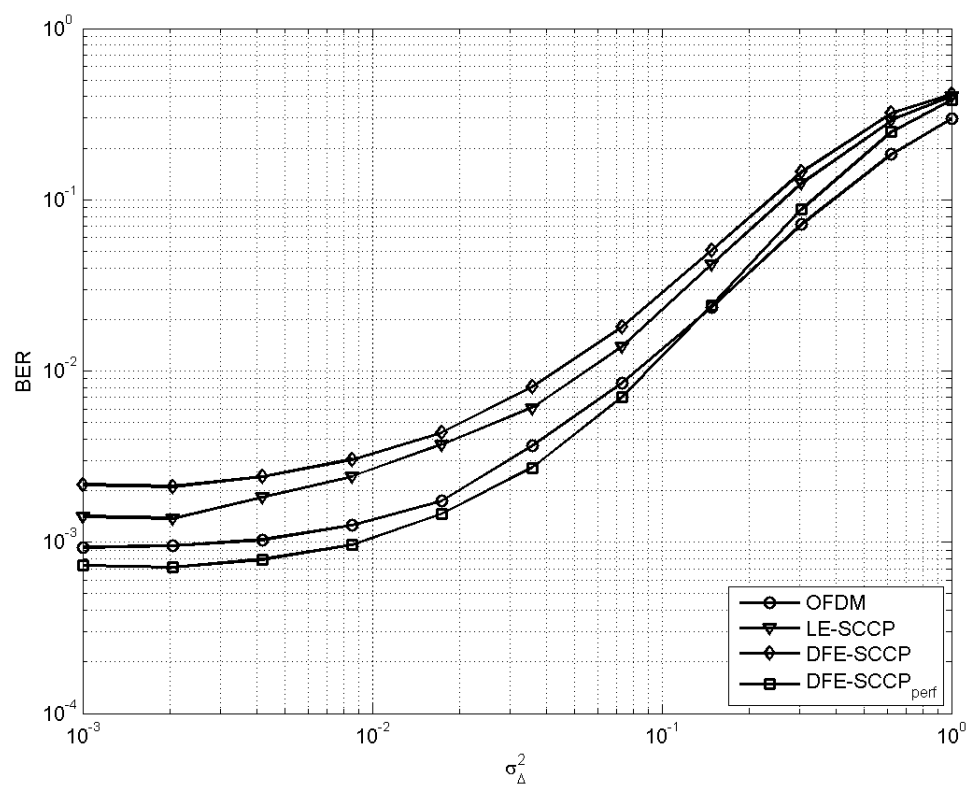

Figura 8.4: Comparação de desempenho. Transmissão QPSK em canal seletivo em frequência com desvanecimento Rayleigh de diversidade $L=3$. Relação $E_{b} / N_{o}$ fixada em $12 \mathrm{~dB}$ e canal com erro de estimação de variância $\sigma_{\Delta}^{2}$. 


\section{Sumário}

Neste capítulo mostrou-se a degradação inserida nos sistemas nas situações em que o canal não é perfeitamente estimado na recepção.

Para o sistema OFDM equalizado com filtro casado, a SNR na saída do equalizador foi obtida através de uma aproximação gaussiana. Então, de posse da SNR, foi possível derivar a expressão da BER para o caso não-codificado. Para o caso codificado, foi utilizada a aproximação da $\mathrm{SNR}_{e f f}$, explorada no capítulo 7, de modo a se obter uma aproximação para a BER. Os resultados mostraram concordância entre a análise teórica e resultados de simulação.

Já para os sistemas SCCP, não foi possível aplicar uma análise teórica nos mesmos moldes daquela aplicada ao sistema OFDM. Os resultados gerados para esse sistema, a partir de simulação Monte Carlo, mostraram uma situação curiosa: a BER não é função decrescente da SNR. Esse comportamento não está presente no sistema OFDM equalizado com filtro casado. Finalmente, comparando ambos os sistemas, percebe-se claramente que o SCCP é mais sensível a erros de estimação do canal que o OFDM. 


\section{Capítulo 9}

\section{Conclusões e Perspectivas}

A dissertação apresentada procurou cobrir algumas das lacunas ainda pouco exploradas no âmbito da comparação entre sistemas OFDM e SCCP.

A fim de permitir tal comparação, foi apresentado, no capítulo 2, juntamente com os conceitos de transmissão por múltiplas portadoras ortogonais, um arcabouço que unifica o tratamento de ambas. Já no capítulo 3, foram apresentadas as estruturas de equalização no domínio da frequência que foram utilizadas ao longo da dissertação.

Uma vez estabelecidos os fundamentos necessários para a comparação nos capítulos anteriores, no capítulo 4 foi dada ênfase a questões envolvendo a ordem da modulação empregada no mapeamento de bits. Explorando características de convexidade de funções obtidas a partir de mapeamentos da função da BER, foi mostrada a degradação sofrida pelo sistema SCCP em relação ao aumento da ordem da modulação. Nesse capítulo, a principal contribuição do trabalho de mestrado foi estender os resultados já publicados para comparação entre sistemas LE-SCCP e OFDM em [24] para comparação entre os sistemas DFE-SCCP e OFDM. Apesar de terem sido obtidos resultados para o caso sem codificação de canal, tais resultados serão refletidos em sistemas em que a codificação se faz presente e podem explicar a degradação de desempenho nos resultados observados em [22] e [26].

Essa concordância entre os resultados simulados e os resultados teóricos gerados para o caso não-codificado estimulou a busca por ferramentas que provessem possibilidades para a análise dos sistemas codificados. Para tanto, no capítulo 5, o parâmetro de cutoff rate já utilizado em [25] para comparação entre sistemas OFDM e LE-SCCP, passou a ser empregado na nossa análise. Neste capítulo, derivam-se as expressões de cutoff rate para os sistemas DFE-SCCP e LE-SCCP, para qualquer modulação do tipo M-QAM. Com as expressões de cutoff rate para os sistemas em estudo, aplicou-se uma análise empregando um formalismo 
matemático semelhante ao apresentado no capítulo 4 e, desse modo, foi possível chegar a algumas conclusões importantes. Primeiramente, mostramos que o sistema DFE-SCCP leva a uma cutoff rate maior do que o sistema OFDM, independentemente da modulação adotada. Já na comparação entre os sistemas LE-SCCP e OFDM, a análise de cutoff rate corroborou os resultados obtidos no caso não-codificado: o aumento na cardinalidade da modulação, levava a uma degradação de desempenho dos sistemas LE-SCCP em relação ao OFDM.

No capítulo 6, foram detalhados três formas distintas de entrelaçamento de bits: o entrelaçador regular, o entrelaçador modular e o entrelação aleatório. A principal contribuição gerada nesse capítulo foi mostrar que a escolha dos parâmetros de entrelaçamento no sistema OFDM precisa ser realizada de uma forma cuidadosa. Tal sensibilidade é ainda maior quando se assume canais estáticos. Os sistemas SCCP, por sua vez, são bem mais robustos que o sistema OFDM em relação ao entrelaçamento. Esse é um fator importante e que ainda tinha sido pouco explorado na literatura. Os resultados referentes a esse capítulo foram publicados em [35] e [36].

Ainda no capítulo 6, vimos que se o entrelaçador de bits estiver corretamente dimensionado e se for utilizado um código com taxa $R=1 / 2$, o desempenho do sistema OFDM é muito próximo do desempenho do sistema DFE-SCCP ideal em todos os casos estudados. A propagação de erros nos sistemas DFE-SCCP fará com que o desempenho do OFDM seja ainda melhor que o do DFE-SCCP quando os sistemas forem implementados em situações reais.

Já no capítulo 7, foi avaliada a $\mathrm{SNR}_{e f f}$ enquanto figura de mérito para os sistemas OFDM. Mostrou-se que em canais seletivos em frequência com desvanecimento Rayleigh por blocos, esse parâmetro resulta em boas aproximações para expressões de BER dos sistemas OFDM codificados.

Finalmente, no capítulo 8 foi analisada a degradação provocada por erros de estimação de canal nos dois sistemas. Nesse capítulo, foi mostrado que o sistema OFDM é consideravelmente mais robusto em relação a erros de estimação de canal do que os sistemas SCCP.

Diante de toda a análise realizada, é possível traçar algumas conclusões mais gerais. Primeiramente, o trabalho deixou evidente que sistemas que empregam constelações de alta cardinalidade tendem a apresentar melhor desempenho se o sinal for transmitido com OFDM. Na verdade, é válido afirmar, inclusive, que o sistema LE-SCCP supera o OFDM apenas nos casos de baixa eficiência espectral. Grande parte das aplicações atuais em comunicações sem-fio, e.g. padrão IEEE 802.11 (a/g/n) e TV digital, utilizam modulações e codificações provendo alta eficiência espectral, limitando o uso dos sistemas LE-SCCP.

De fato, o problema do OFDM reside em sua dependência com as condições de codificação de canal. O código precisa ter um bom poder de correção e, aliado a isso, ainda é 
necessário que os bits sejam entrelaçados do forma adequada. A limitação do código não é tão restritiva, uma vez que atualmente os sistemas de comunicação costumam aplicar códigos corretores de erros suficientemente eficientes para que o desempenho do OFDM seja satisfatório. A limitação do entrelaçamento, por sua vez, é mais problemática por sua condição de otimalidade ser uma função direta do canal de transmissão. Esse fato impossibilita o projeto de entrelaçadores ótimos para o OFDM sem que haja conhecimento de canal na transmissão. E, caso houvesse conhecimento de canal, a abordagem para o problema teria uma outra conotação, baseada em algoritmos de waterfilling, eliminando tal dependência com entrelaçamento.

Enquanto perspectivas, um fato não analisado e que mereceria atenção diz respeito aos sistemas MIMO. Nesse caso, o OFDM conseguiria explorar ramos de diversidade mesmo sem código. Entretanto, aliado a isso, vem a interferência entre os vários sinais OFDM em cada uma das antenas. Tal interferência pode ser mitigada utilizando técnicas similares às utilizadas para combater a ISI nos sistemas SCCP. 



\section{Apêndice A}

\section{Tratamento de desigualdades: Hardy, Littlewood e Pólya}

No clássico livro Inequalities publicado originalmente em 1934 [49], os matemáticos britânicos G. H. Hardy e J. E. Littlewood e o húngaro G. Pólya propuseram um método simples e elegante para demonstrar uma importante família de desigualdades. A fim de descrever o método, iremos provar o seguinte teorema:

Teorema 1. Sejam duas médias $\mathcal{M}_{1}$ e $\mathcal{M}_{2}$, tais que existam funções $f(x)$, crescente, e $g(x)$, tais que:

$$
\begin{aligned}
& \mathcal{M}_{1}=f^{-1}\left(\frac{1}{N} \sum_{k=0}^{N-1} f\left(x_{k}\right)\right) \\
& \mathcal{M}_{2}=g^{-1}\left(\frac{1}{N} \sum_{k=0}^{N-1} g\left(x_{k}\right)\right)
\end{aligned}
$$

em que $f^{-1}(x)$ é a função inversa de $f(x)$, i.e. $f\left(f^{-1}(x)\right)=x$. A mesma observação vale para $g(x)$. Então, se a função $t(x)=f\left(g^{-1}(x)\right)$ for convexa, $\mathcal{M}_{1} \geq \mathcal{M}_{2}$.

Demonstração. Queremos comparar $\mathcal{M}_{1}$ e $\mathcal{M}_{2}$. Por hipótese, $f(x)$ é uma função monotonicamente crescente. Logo, $f\left(\mathcal{M}_{1}\right) \geq f\left(\mathcal{M}_{2}\right) \Rightarrow \mathcal{M}_{1} \geq \mathcal{M}_{2}$. Apliquemos a função $f(x)$ em $\mathcal{M}_{1}$ 
e $\mathcal{M}_{2}$ :

$$
\begin{array}{r}
f\left(\mathcal{M}_{1}\right)=\frac{1}{N} \sum_{k=0}^{N-1} f\left(x_{k}\right) \\
f\left(\mathcal{M}_{2}\right)=f\left(g^{-1}\left(\frac{1}{N} \sum_{k=0}^{N-1} g\left(x_{k}\right)\right)\right)
\end{array}
$$

Reescrevendo as eqs.(A.1) e (A.2) em termos da função $t(x)$, temos que:

$$
\begin{array}{r}
f\left(\mathcal{M}_{1}\right)=\frac{1}{N} \sum_{k=0}^{N-1} t\left(g\left(x_{k}\right)\right) \\
f\left(\mathcal{M}_{2}\right)=t\left(\frac{1}{N} \sum_{k=0}^{N-1} g\left(x_{k}\right)\right)
\end{array}
$$

e como $t(x)$ é convexa, por hipótese, a Desigualdade de Jensen garante que:

$$
\frac{1}{N} \sum_{k=0}^{N-1} t\left(g\left(x_{k}\right)\right) \geq t\left(\frac{1}{N} \sum_{k=0}^{N-1} g\left(x_{k}\right)\right) \leftrightarrow \mathcal{M}_{1} \geq \mathcal{M}_{2}
$$

É imediato mostrar que se a função $f(x)$ for decrescente, a condição $\mathcal{M}_{1} \geq \mathcal{M}_{2}$ é válida para $t(x)$ côncava.

Esse teorema é extremamente poderoso e pode ser aplicada para demonstrar uma série de desigualdades importantes. A título de exemplo, iremos demonstrar que a média aritmética (amean) é maior que a média geométrica (geomean) que é maior que a média harmônica (harmmean).

Teorema 2. Seja amean $=\frac{1}{N} \sum_{k=0}^{N-1} x_{k}$ e geomean $=\prod_{k=0}^{N-1} x_{k}^{\frac{1}{N}}$, em que a $x_{k}, k=0, \ldots, k=$ $N-1$ é uma sequência de números reais não-negativos. Então, amean $\geq$ geomean, para qualquer sequência $\left.x_{k}\right|_{k=0} ^{N-1}$.

Demonstração. Note que geomean $=\prod_{k=0}^{N-1} x_{k}^{\frac{1}{N}}$ pode ser escrita como:

$$
\text { geomean }=\exp \left(\frac{1}{N} \sum_{k=0}^{N-1} \log \left(x_{k}\right)\right)
$$


Definindo $f(x)=\log (x)$, temos:

$$
\text { geomean }=f^{-1}\left(\frac{1}{N} \sum_{k=0}^{N-1} f\left(x_{k}\right)\right)
$$

De modo semelhante podemos definir $g(x)=x$ e escrever:

$$
\text { amean }=g^{-1}\left(\frac{1}{N} \sum_{k=0}^{N-1} g\left(x_{k}\right)\right)
$$

A função $t(x)=f\left(g^{-1}(x)\right)=\log (x)$ é côncava, já que $\frac{d^{2}}{d x^{2}} t(x)=-\frac{1}{x^{2}} \leq 0$. Como $f(x)$ é crescente podemos aplicar o teorema 1 , concluindo que amean $\geq$ geomean

A prova da desigualdade entre as médias geométrica e harmônica pode ser feita como se segue:

Teorema 3. Seja harmmean $=\frac{1}{\frac{1}{N} \sum_{k=0}^{N-1} \frac{1}{x_{k}}}$ e geomean $=\prod_{k=0}^{N-1} x_{k}^{\frac{1}{N}}$, em que a $x_{k}, k=$ $0, \ldots, k=N-1$ é uma sequência de números reais não-negativos. Então, geomean $\geq$ harmmean, para qualquer sequência $\left.x_{k}\right|_{k=0} ^{N-1}$.

Demonstração. A partir da prova anterior, temos:

$$
\text { geomean }=f^{-1}\left(\frac{1}{N} \sum_{k=0}^{N-1} f\left(x_{k}\right)\right)
$$

$\operatorname{com} f(x)=\log (x)$ Podemos escrever a média harmônica como:

$$
\text { harmmean }=g^{-1}\left(\frac{1}{N} \sum_{k=0}^{N-1} g\left(x_{k}\right)\right)
$$

em que $g(x)=1 / x$. A função $t(x)=f\left(g^{-1}(x)\right)=\log \left(\frac{1}{x}\right)$ é convexa, já que sua segunda derivada é estritamente positiva $\frac{d^{2}}{d x^{2}} t(x)=\frac{1}{x^{2}} \geq 0$. Aplicando o teorema 1 , concluímos que geomean $\geq$ harmmean 



\section{Apêndice B}

\section{Análise da função $b(y)$}

Vejamos para quais valores de $y$ a função:

$$
b(y)=1-\frac{\beta e^{y}}{2}\left(1+\frac{1}{\beta\left(e^{y}-1\right)}\right)
$$

assume valores positivos.

A partir da expressão acima, temos que:

$$
b(y) \geq 0 \leftrightarrow 2 \beta\left(e^{y}-1\right) \geq \beta e^{y}\left(\beta\left(e^{y}-1\right)+1\right)
$$

ou ainda:

$$
\beta e^{2 y}-(\beta+1) e^{y}+2 \leq 0
$$

o que implica em:

$$
\Delta=(1+\beta)^{2}-8 \beta \geq 0
$$

e dessa forma:

$$
\beta \leq 3-2 \sqrt{2} \quad \text { ou } \quad \beta \geq 3+2 \sqrt{2}
$$

Nessas condições, os valores de $y$ que satisfazem (B.3) são dados por:

$$
\log \left(\frac{(\beta+1)-\sqrt{(1+\beta)^{2}-8 \beta}}{2 \beta}\right) \leq y \leq \log \left(\frac{(\beta+1)+\sqrt{(1+\beta)^{2}-8 \beta}}{2 \beta}\right)
$$





\section{Referências Bibliográficas}

[1] S. B. Weinstein, "The history of orthogonal frequency-division multiplexing," Communications Magazine, IEEE, vol. 47, no. 11, pp. 26-35, November 2009.

[2] M. Schwartz, "The origins of carrier multiplexing: Major George Owen Squier and AT\&T," Communications Magazine, IEEE, vol. 46, no. 5, pp. 20-24, May 2008.

[3] R. W. Chang, "Synthesis of band-limited orthogonal signals for multichannel data transmission," Bell Sys. Tech. J., vol. 45, 1966.

[4] M. Zimmerman and A. Kirsch, "The AN/GSC-10 (KATHRYN) variable rate data modem for hf radio," Communication Technology, IEEE Transactions on, vol. 15, pp. 197-204, 1967.

[5] G. Porter, "Error distribution and diversity performance of a frequency-differential PSK HF modem," Communication Technology, IEEE Transactions on, vol. 16, pp. 567-275, 1968.

[6] J. W. Cooley and J. W. Tukey, "An algorithm for the machine calculation of complex Fourier series," Mathematics of Computation, vol. 19, pp. 297-301, 1965.

[7] S. Weinstein and P. Ebert, "Data transmission by frequency-division multiplexing using the discrete Fourier transform," Communication Technology, IEEE Transactions on, vol. 19, no. 5, pp. 628-634, October 1971.

[8] K. Sistanizadeh, P. Chow, and J. Cioffi, "Multi-tone transmission for asymmetric digital subscriber lines (ADSL)," in IEEE International Conference on Communications, 1993. ICC 93., May 1993, vol. 2, pp. 756-760.

[9] U. Reimers, "DVB-T: the COFDM-based system for terrestrial television," Electronics \& Communication Engineering Journal, vol. 9, no. 1, pp. 28-32, Feb 1997. 
[10] S. Nakahara, M. Okano, M. Takada, and T. Kuroda, "Digital transmission scheme for ISDB-T and reception characteristics of digital terrestrial television broadcasting system in Japan," Consumer Electronics, IEEE Transactions on, vol. 45, no. 3, pp. 563-570, Aug 1999.

[11] IEEE-SA, IEEE Standard for Information Technology - Telecommunications and information exchange between systems - Local and metropolitan area networks- Specific requirements. Part 11: Wireless LAN Medium Access Control (MAC) and Physical Layer (PHY) Specifications, June 2007.

[12] Chuen-Ching Wang and Yih-Chuan Lin, "An efficient FFT processor for DAB receiver using circuit-sharing pipeline design," Broadcasting, IEEE Transactions on, vol. 53, no. 3, pp. 670-677, Sept. 2007.

[13] T. M. Cover and J. A. Thomas, Elements of Information Theory, Wiley, 2006.

[14] D. Zanatta, L. Féty, and M. Terré, "Water-filling for cyclic prefixed single carrier transmission and MMSE receiver," in European Wireless Conference, 200\%. EW200\%., July 2007, pp. 181-185.

[15] T. J. Willink and P. H. Wittke, "Optimization and performance evaluation of multicarrier transmission," Information Theory, IEEE Transactions on, vol. 43, no. 2, pp. 426-440, Mar 1997.

[16] H. Sari, G. Karam, and I. Jeanclaude, "Transmission techniques for digital terrestrial TV broadcasting," IEEE Communications Magazine, vol. 33, no. 2, pp. 100-109, Feb. 1995.

[17] M. E. Austin, "Decision feedback equalization for digital communication over dispersive channels," MIT Research Laboratory of Electronics Technical Report, vol. 461, Aug. 1967.

[18] K. Berberidis and J. Palicot, "A frequency-domain decision feedback equalizer for multipath echo cancellation," in Global Telecommunications Conference, 1995. GLOBECOM '95., IEEE, Nov 1995, vol. 1, pp. 98-102.

[19] J. J. Shynk, "Frequency domain and multirate adaptive filtering," IEEE Signal Processing Magazine, vol. 9, no. 1, pp. 14-37, Jan. 1992. 
[20] N. Benvenuto and S. Tomasin, "On the comparison between OFDM and single carrier modulation with a DFE using a frequency-domain feedforward filter," IEEE Trans. On Communications, vol. 50, no. 6, June 2002.

[21] D. D. Falconer and S. L. Ariyavisitakul, "Broadband wireless using single carrier and frequency domain equalization," in Wireless Personal Multimedia Communications, 2002. The 5th International Symposium on, Oct. 2002, vol. 1, pp. 27-36.

[22] D. Falconer, S. L. Ariyavisitakul, A. Benjamin-Seeyar, and B. Edison, "Frequency domain equalization for single-carrier broadband wireless systems," IEEE Communications Magazine, vol. 40, pp. 58-66, April 2002.

[23] Yuan-Pei Lin and See-May Phoong, "MMSE OFDM and prefixed single carrier systems: BER analysis," Acoustics, Speech, and Signal Processing, 2003. Proceedings. (ICASSP '03). 2003 IEEE International Conference on, vol. 4, pp. IV-229-32, April 2003.

[24] Yuan-Pei Lin and See-May Phoong, "BER minimized OFDM systems with channel independent precoders," Signal Processing, IEEE Transactions on, vol. 51, no. 9, pp. 2369-2380, Sept. 2003.

[25] V. Aue, G. P. Fettweis, and R. Valenzuela, "A comparison of the performance of linearly equalized single carrier and coded OFDM over frequency selective fading channels using the random coding technique," in Proc. Int. Conf. Communications (ICC), Atlanta, 1998, vol. 2, pp. $753-757$.

[26] H. Witschnig, G. Ossberger, A. Springer, A. Koppler, L. Maurer, M. Huemer, and R. Weigel, "The effect of blockwise transmission on higher-order modulation schemes for SC/FDE," in Wireless Personal Multimedia Communications, 2002. The 5th International Symposium on, Oct. 2002, vol. 3, pp. 1323-1327.

[27] Zhiqiang Liu, "Maximum diversity in single-carrier frequency-domain equalization," Information Theory, IEEE Transactions on, vol. 51, no. 8, pp. 2937-2940, Aug. 2005.

[28] M. Ghogho, V.P. Gil-Jimenez, and A. Swami, "Multipath diversity and coding gains of cyclic-prefixed single carrier systems," in Acoustics, Speech and Signal Processing, 2009. ICASSP 2009. IEEE International Conference on, April 2009, pp. 2837-2840.

[29] A. Medles and D.T.M. Slock, "Decision-feedback equalization achieves full diversity for finite delay spread channels," in Information Theory, 2004. ISIT 2004. Proceedings. International Symposium on, June-2 July 2004, p. 99. 
[30] J.M. Cioffi, G.P. Dudevoir, M.V. Eyuboglu, and Jr. Forney, G.D., "MMSE decisionfeedback equalizers and coding. II. Coding results," Communications, IEEE Transactions on, vol. 43, no. 10, pp. 2595-2604, Oct 1995.

[31] S. K. Wilson and J. M. Cioffi, "A comparison of a single-carrier system using a DFE and a coded OFDM system in a broadcast rayleigh-fading channel," Proceedings of IEEE Int. Symp. on Information Theory, 1995, pp. 335-, Sep 1995.

[32] A. Gusmão, R. Dinis, J. Conceição, and N. Esteves, "Comparison of two modulation choices for broadband wireless communications," in Vehicular Technology Conference Proceedings, 2000. VTC 2000-Spring Tokyo. 2000 IEEE 51st, 2000, vol. 2, pp. 13001305.

[33] F. Pancaldi, G. Vitetta, R. Kalbasi, N. Al-Dhahir, M. Uysal, and H. Mheidat, "Singlecarrier frequency domain equalization," IEEE Signal Processing Magazine, vol. 25, pp. 37-56, 2008.

[34] R. Kalbasi, D. D. Falconer, A. H. Banihashemi, and R. Dinis, "A comparison of frequency-domain block MIMO transmission systems," Vehicular Technology, IEEE Transactions on, vol. 58, no. 1, pp. 165-175, Jan. 2009.

[35] A. S. Paula and C. M. Panazio, "A Robustness and Performance Comparison Between Cyclic Prefixed Single-Carrier and OFDM Systems," in IWT, International Workshop on Telecommunicatios, 2009.

[36] A. S. Paula e C. M. Panazio, "Efeito do entrelaçador na comparação de desempenho de sistemas OFDM e SCCP," in Simpósio Brasileiro de Telecomunicações, SBrT, Blumenau, Brasil, 2009.

[37] Yuan-Pei Lin and See-May Phoong, "OFDM transmitters: analog representation and DFT-based implementation," Signal Processing, IEEE Transactions on, vol. 51, no. 9, pp. 2450-2453, Sept. 2003.

[38] A. V. Oppenheim and R.W. Schafer, Discrete-Time Signal Processing, Prentice Hall, 1999.

[39] A. Peled and A. Ruiz, "Frequency domain data transmission using reduced computational complexity algorithms," Acoustics, Speech, and Signal Processing, IEEE International Conference on ICASSP '80., vol. 5, pp. 964-967, Apr 1980.

[40] R. A. Horn, Matrix Analysis, Cambridge University Press, 1987. 
[41] D. Falconer, S. L. Ariyavistakul, A. Benyamin-Seeyar, and B. Edison, White Paper: Frequency Domain Equalization for Single-Carrier Broadband Wireless Systems, 2002.

[42] R. Lopez-Valcarce, "Realizable linear and decision feedback equalizers: properties and connections," Signal Processing, IEEE Transactions on, vol. 52, no. 3, pp. 757-773, March 2004.

[43] H. Witschnig, T. Mayer, A. Springer, L. Maurer, M. Huemer, and R. Weigel, "The advantages of a known sequence versus cyclic prefix in a SC/FDE system," in Wireless Personal Multimedia Communications, 2002. The 5th International Symposium on, Oct. 2002, vol. 3, pp. 1328-1332 vol.3.

[44] G. Huang, A. Nix, and S. Armour, "Decision feedback equalization in SC-FDMA," in Personal, Indoor and Mobile Radio Communications, 2008. PIMRC 2008. IEEE 19th International Symposium on, Sept. 2008, pp. 1-5.

[45] J. Ashley, M. Blaum, B. Marcus, and C.M. Melas, "Performance and error propagation of two DFE channels," Magnetics, IEEE Transactions on, vol. 33, no. 5, pp. 2773-2775, Sep 1997.

[46] A. Goupil and J. Palicot, "Markovian model of the error probability density and application to the error propagation probability computation of the weighted, decision feedback equalizer," in Acoustics, Speech, and Signal Processing, 2001. Proceedings. (ICASSP '01). 2001 IEEE International Conference on, 2001, vol. 4, pp. 2525-2528.

[47] J. E. Smee and N. C. Beaulieu, "Error-rate evaluation of linear equalization and decision feedback equalization with error propagation," Communications, IEEE Transactions on, vol. 46, no. 5, pp. 656-665, May 1998.

[48] H. Li and T. Adali, "Complex-valued adaptive signal processing using nonlinear functions," Journal on Advances in Signal Processing, Special issue on Emerging Machine Learning Techniques in Signal Processing, 2008.

[49] G. H. Hardy, J. E. Littlewood, and G. Pólya, Inequalities, Cambridge, 1952.

[50] B. Devillers, J. Louveaux, and L. Vandendorpe, "About the diversity in cyclic prefixed single-carrier systems," Physical Communication, Elsevier, vol. 1, pp. 266-276, Dec. 2008.

[51] J. Salz, "Optimum mean-square decision feedback equalization," Bell Syst. Tech. J., vol. 52, pp. 1341-1373, Oct. 1973. 
[52] J. Proakis and M. Salehi, Digital Communications, Mc Graw-Hill, 5 edition, 2008.

[53] D. Tse and P. Viswanath, Fundamentals of Wireless Communication, Cambridge, 2005.

[54] J. Wozencraft and I. M. Jacobs, Principles of Communication Engineering, Wiley, 1965.

[55] J. Wozencraft and R. Kennedy, "Modulation and demodulation for probabilistic coding," Information Theory, IEEE Transactions on, vol. 12, no. 3, pp. 291-297, Jul 1966.

[56] S. Ramseier, "Shuffling bits in time and frequency: an optimum interleaver for OFDM," in International Conference on Communications - ICC, 2003.

[57] G. Caire, G. Taricco, and E. Biglieri, "Bit-interleaved coded modulation," Information Theory, IEEE Transactions on, vol. 44, no. 3, pp. 927-946, May 1998.

[58] G. Ungerboeck, "Channel coding with multilevel/phase signals," Information Theory, IEEE Transactions on, vol. 28, no. 1, pp. 55-67, Jan 1982.

[59] E. Biglieri, D. Divsalar, P. J. MacLane, and M. K. Simon, Introduction to Trellis-Coded Modulation with Application, Macmillan, 1991.

[60] G. C. Clark and J. B. Cain, Error-Correction Coding for Digital Communications, Plenum Press, New York, 1981.

[61] D. Burton, Elementary Number Theory, McGraw-Hill, 2005.

[62] H. R. Sadjadpour, N. J. A. Sloane, M. Salehi, and G. Nebe, "Interleaver design for turbo codes," JSAC- Journal on Selected Areas in Communications, vol. 19, pp. 831$837,2000$.

[63] C. M. Panazio and J. M. T. Romano, "Performance of joint space-time equalization and decoding techniques for wireless systems," in International Telecommunications Symposium-ITS, Natal, Brazil, 2002.

[64] S. Lin and D. J. Costello, Error Control Coding, Prentice Hall, 2004.

[65] Y. Nasser, J. F. Helard, and M. Crussiere, "Bit error rate prediction of coded MIMOOFDM systems," in Signal Processing Advances in Wireless Communications, 2008. SPAWC 2008. IEEE 9th Workshop on, July 2008, pp. 181-185. 
[66] K. Brueninghaus, D. Astely, T. Salzer, S. Visuri, A. Alexiou, S. Karger, and G.-A. Seraji, "Link performance models for system level simulations of broadband radio access systems," in Personal, Indoor and Mobile Radio Communications, 2005. PIMRC 2005. IEEE 16th International Symposium on, Sept. 2005, vol. 4, pp. 2306-2311.

[67] L. Wan, S. Tsai, and M. Almgren, "A fading-insensitive performance metric for a unified link quality model," in Wireless Communications and Networking Conference, 2006. WCNC 2006. IEEE, April 2006, vol. 4, pp. 2110-2114.

[68] E. Westman, "Calibration and evaluation of the exponential effective SINR mapping (EESM) in 802.16," M.S. thesis, KTH Electrical Engineering, 2007.

[69] S. M. Kay, Fundamentals of Statical Signal Processing, Volume II: Detection Theory, Prentice Hall, 1998. 